\title{
A multicomponent synthesis of spiroisoxazolines
}

\author{
Mauro F. A. Adamo, Donato Donati, Eleanor F. Duffy and Piero Sarti-Fantoni
}

Table of Contents

S2: General experimental.

S3-9: spectroscopic data for compounds 5d-p and 3d-m.

S10-26: ${ }^{1} \mathrm{H}-\mathrm{NMR}$ spectra showing the epimerisation of 5a to 4-epi-5a.

S27-39: ${ }^{1} \mathrm{H}-\mathrm{NMR}$ of compounds $\mathbf{5 b - p}$.

S40-52: ${ }^{1} \mathrm{H}-\mathrm{NMR}$ of compounds 3a-m.

S53-54: ${ }^{1} \mathrm{H}-\mathrm{NMR}(400 \mathrm{MHz})$ and peak assignments of compounds 5a and 4-epi-5a.

S55-58: ${ }^{1} \mathrm{H}-{ }^{1} \mathrm{H}$ correlation and ${ }^{1} \mathrm{H}_{-}{ }^{13} \mathrm{C}$ correlation NMR of compound 5a and 4-epi-5a.

S59: n.O.e. experiments for the assignment of 5a and 4-epi-5a (reference n. 9). 
General experimental: ${ }^{1} \mathrm{H}$ and ${ }^{13} \mathrm{C}$ Spectra were recorded on a 200 or a $400 \mathrm{MHz}$ spectrometers at ambient temperatures. ${ }^{1} \mathrm{H}$ NMR spectral assignments are supported by ${ }^{1} \mathrm{H}-{ }^{1} \mathrm{H}$ COSY and ${ }^{13} \mathrm{C}-{ }^{1} \mathrm{H} \operatorname{COSY}$ where necessary. For ${ }^{1} \mathrm{H}$ NMR recorded in $\mathrm{CDCl}_{3}$ chemical shifts $\left(\delta_{\mathrm{H}}\right)$ are quoted in parts per million $(\mathrm{ppm})$ and are referenced to the residual solvent peak. The following abbreviations are used: s, singlet, $\mathrm{d}$, doublet, t, triplet, dd, doublet of doublets, dt, doublet of triplets, tt, triplet of triplets, m, multiplet and br, broad. Coupling constants $(J)$ were recorded in $\operatorname{Hertz}(\mathrm{Hz})$ to the nearest $0.5 \mathrm{~Hz}$. Carbon spectra are supported by DEPT analysis where necessary. Infrared (IR) spectra were recorded as thin films between $\mathrm{NaCl}$ plates. Absorption maximum $\left(v_{\max }\right)$ was reported in wave numbers $\left(\mathrm{cm}^{-1}\right)$ and only selected peaks are reported. The following abbreviations are used: w, weak, m, medium, s, strong and br, broad.

Flash chromatography was carried out using silica gel $60(0.040-0.063 \mathrm{~mm}, 230-400 \mathrm{mesh})$ as the stationary phase. Thin layer chromatography was carried out on aluminium backed plates pre-coated with silica gel 60, which were visualized by quenching of u.v. fluorescence $\left(\lambda_{\max }=254 \mathrm{~nm}\right)$ or by staining with either $10 \% \mathrm{w} / \mathrm{v}$ ammonium molybdate in $2 \mathrm{M}$ sulphuric acid or basic potassium permanganate solution (followed by heat) as appropriate. Retention factors $\left(\mathrm{R}_{\mathrm{f}}\right)$ are reported to \pm 0.5 . 
Spectroscopic data for compounds 5d-p and 3d-m:

8-Acetyl-3-methyl-4-nitro-9-(4-nitro-phenyl)-1-oxa-2-aza-spiro[4.5]dec-2-en-7-one 5d: colourless solid (932 mg, 83\% yield) m.p.181-2 ${ }^{\circ} \mathrm{C}$ (ethanol); $v_{\max }\left(\right.$ Film) $/ \mathrm{cm}^{-1}: 1720 \mathrm{~s}, 1560 \mathrm{~s} ;{ }^{1} \mathrm{H}-\mathrm{NMR} \delta:(200$ $\left.\mathrm{MHz}, \mathrm{CD}_{3} \mathrm{COCD}_{3}\right) 7.96(2 \mathrm{H}, \mathrm{d}, J=8), 7.48(2 \mathrm{H}, \mathrm{d}, J=8), 5.80(1 \mathrm{H}, \mathrm{s}), 4.35(1 \mathrm{H}, \mathrm{d}, J=12), 3.70(1 \mathrm{H}$, $\mathrm{td}, J=12, J=4), 3.05(1 \mathrm{H}, \mathrm{d}, J=14), 2.53-2.43(2 \mathrm{H}, \mathrm{m}), 1.90(3 \mathrm{H}, \mathrm{s}), 1.81(3 \mathrm{H}, \mathrm{s}), 1.74-1.65(1 \mathrm{H}, \mathrm{m})$;

${ }^{13} \mathrm{C}-\mathrm{NMR} \delta$ : (80MHz, $\left.\mathrm{CD}_{3} \mathrm{COCD}_{3}\right)$ 203.7, 201.9, 200.8, 153.3, 153.1, 146.5, 146.4, 139.5, 138.9, 128.8, $128.1,123.7,123.3,97.7,97.5,88.2,66.6,66.1,48.7,44.6,41.1,40.7,40.5,35.6,29.5,10.3$. HRMS found: $\mathrm{MH}^{+} 376.1143, \mathrm{C}_{17} \mathrm{H}_{18} \mathrm{~N}_{3} \mathrm{O}_{7}$ requires 376.1145; $m / z: 376\left(85 \%, \mathrm{MH}^{+}\right)$.

3-Methyl-4-nitro-7-(4-nitro-phenyl)-9-oxo-1-oxa-2-aza-spiro[4.5]dec-2-ene-8-carboxylic acid ethyl ester 5e: colorless solid (862 mg, $71 \%$ yield), m.p. $152-3{ }^{\circ} \mathrm{C}$ (ethanol), $v_{\max }\left(\right.$ Film) $/ \mathrm{cm}^{-1}: 1731 \mathrm{~s}, 1570 \mathrm{~s}$; ${ }^{1} \mathrm{H}-\mathrm{NMR} \delta$ : (200 MHz, $\left.\mathrm{CDCl}_{3}\right) 8.20(2 \mathrm{H}, \mathrm{d}, J=8), 7.47(2 \mathrm{H}, \mathrm{d}, J=8), 5.39(1 \mathrm{H}, \mathrm{s}), 4.20-3.90(3 \mathrm{H}, \mathrm{m})$, $3.74(1 \mathrm{H}, \mathrm{d}, J=14), 2.86(1 \mathrm{H}, \mathrm{d}, J=16), 2.76(1 \mathrm{H}, \mathrm{d}, J=16), 2.31-2.08(2 \mathrm{H}, \mathrm{m}), 2.17(3 \mathrm{H}, \mathrm{s}), 1.13$ $(3 \mathrm{H}, \mathrm{t}, J=7) ;{ }^{13} \mathrm{C}-\mathrm{NMR} \delta:\left(80 \mathrm{MHz}, \mathrm{CD}_{3} \mathrm{COCD}_{3}\right) 197.2,166.9,152.2,151.4,146.1,145.2,138.8$, $138.1,128.9,123.5,122.1,97.6,97.3,88.1,62.1,61.8,59.2,48.0,44.2,42.5,42.1,41.8,37.8,12.5$, 10.1; HRMS found: $\mathrm{MH}^{+} 406.1249, \mathrm{C}_{18} \mathrm{H}_{20} \mathrm{~N}_{3} \mathrm{O}_{8}$ requires 406.1250; $m / z: 406\left(100 \%, \mathrm{MH}^{+}\right)$.

3-Methyl-4-nitro-9-(4-nitro-phenyl)-1-oxa-2-aza-spiro[4.5]dec-2-en-7-one 5f: colourless solid (779 $\mathrm{mg}, 78 \%$ yield), m.p. $148-9{ }^{\circ} \mathrm{C}$ (ethanol), $v_{\max }\left(\right.$ Film) $/ \mathrm{cm}^{-1}: 1715 \mathrm{~s}, 1575 \mathrm{~s} ;{ }^{1} \mathrm{H}-\mathrm{NMR} \delta:(200 \mathrm{MHz}$, $\left.\mathrm{CDCl}_{3}\right) 7.99(2 \mathrm{H}, \mathrm{d}, J=8), 7.49(2 \mathrm{H}, \mathrm{d}, J=8), 5.78(1 \mathrm{H}, \mathrm{s}), 3.37(1 \mathrm{H}, \mathrm{td}, J=14, J=4), 2.94-2.66(2 \mathrm{H}$, m), 2.41-2.04 (3H, m), $1.89(3 \mathrm{H}, \mathrm{s})$ 1.84-1.62 (1H, m); ${ }^{13} \mathrm{C}-\mathrm{NMR} \delta:\left(80 \mathrm{Mhz}, \mathrm{CD}_{3} \mathrm{COCD}_{3}\right)$ 202.3, 202.1, $150.3,150.2,146.7,141.2,127.9,127.8,123.4,123.2,98.1,97.7,88.5,48.2,46.0,45.6,43.2,39.3$, 38.3, 38.1, 36.2, 10.7. HRMS found: $\mathrm{MH}^{+} 334.1038, \mathrm{C}_{15} \mathrm{H}_{16} \mathrm{~N}_{3} \mathrm{O}_{6}$ requires 334.1039; m/z: 334 (100\%, $\left.\mathrm{MH}^{+}\right)$. 
8-Acetyl-9-(4-chloro-phenyl)-3-methyl-4-nitro-1-oxa-2-aza-spiro[4.5]dec-2-en-7-one 5g: colourless solid (873 mg, 80\% yield), m.p. $184-5{ }^{\circ} \mathrm{C}$ (ethanol); $v_{\max }\left(\right.$ Film) $/ \mathrm{cm}^{-1}: 1725 \mathrm{~s}, 1560 \mathrm{~s} ;{ }^{1} \mathrm{H}-\mathrm{NMR} \delta:(200$ $\left.\mathrm{MHz}, \mathrm{CDCl}_{3}\right) 7.29(2 \mathrm{H}, \mathrm{d}, J=8), 7.15(2 \mathrm{H}, \mathrm{d}, J=8), 5.36(1 \mathrm{H}, \mathrm{s}), 3.85(1 \mathrm{H}, \mathrm{td}, J=12, J=4), 3.75$ $(1 \mathrm{H}, \mathrm{d}, J=12), 2.84-2.75(2 \mathrm{H}, \mathrm{m}), 2.26-2.01(2 \mathrm{H}, \mathrm{m}), 2.16(3 \mathrm{H}, \mathrm{s}), 2.01(3 \mathrm{H}, \mathrm{s}) ;{ }^{13} \mathrm{C}-\mathrm{NMR} \delta:(80 \mathrm{MHz}$, $\left.\mathrm{CD}_{3} \mathrm{COCD}_{3}\right)$ 202.8, 201.9, 201.8, 150.4, 150.2, 140.2, 140.1, 132.0, 131.9, 128.8, 128.7, 128.3, 97.8, 97.4, 88.0, 66.3, 65.9, 48.6, 44.6, 40.6, 40.3, 40.1, 35.9, 29.6, 10.7. HRMS found: $\mathrm{MH}^{+} 365.0902$, $\mathrm{C}_{17} \mathrm{H}_{18} \mathrm{ClN}_{2} \mathrm{O}_{5}$ requires 365.0904; $m / z$ : $365\left(100 \%, \mathrm{MH}^{+}\right)$.

7-(4-Chloro-phenyl)-3-methyl-4-nitro-9-oxo-1-oxa-2-aza-spiro[4.5]dec-2-ene-8-carboxylic acid ethyl ester 5h: colourless solid (815 mg, 69\% yield), m.p. $151-2^{\circ} \mathrm{C}$ (ethanol), $v_{\max }\left(\right.$ Film) $/ \mathrm{cm}^{-1}: 1728 \mathrm{~s}$, 1576s; ${ }^{1} \mathrm{H}-\mathrm{NMR} \delta:\left(200 \mathrm{MHz}, \mathrm{CDCl}_{3}\right) 7.07(2 \mathrm{H}, \mathrm{d}, J=8), 6.95(2 \mathrm{H}, \mathrm{d}, J=8), 5.14(1 \mathrm{H}, \mathrm{s}), 3.89-3.76$ $(2 \mathrm{H}, \mathrm{m}), 3.76-3.53(1 \mathrm{H}, \mathrm{m}), 3.43(1 \mathrm{H}, \mathrm{d}, J=13), 2.58(1 \mathrm{H}, \mathrm{d}, J=15), 2.48(1 \mathrm{H}, \mathrm{dd}, J=15, J=2), 2.05-$ $2.91(2 \mathrm{H}, \mathrm{m}), 1.91(3 \mathrm{H}, \mathrm{s}), 0.86(3 \mathrm{H}, \mathrm{t}, J=7) ;{ }^{13} \mathrm{C}-\mathrm{NMR} \delta:\left(80 \mathrm{MHz}, \mathrm{CDCl}_{3}\right)$ 197.0, 196.6, 166.0, 148.8, $148.2,137.4,132.6,128.0,127.5,97.6,97.3,86.9,61.5,61.3,60.2,48.0,44.3,39.9,39.8,39.7,34.9$, 12.8, 10.9. HRMS found: $\mathrm{MH}^{+} 395.1009, \mathrm{C}_{18} \mathrm{H}_{20} \mathrm{ClN}_{2} \mathrm{O}_{6}$ requires $395.1001 ; m / z: 395\left(80 \%, \mathrm{MH}^{+}\right)$.

9-(4-Chloro-phenyl)-3-methyl-4-nitro-1-oxa-2-aza-spiro[4.5]dec-2-en-7-one 5i: colourless solid (830 mg, 86\% yield), m.p. $164-5^{\circ} \mathrm{C}$ (ethanol), $v_{\max }\left(\right.$ Film)/cm ${ }^{-1}: 1720 \mathrm{~s}, 1572 \mathrm{~s} ;{ }^{1} \mathrm{H}-\mathrm{NMR} \delta:(200 \mathrm{MHz}$, $\left.\mathrm{CD}_{3} \mathrm{COCD}_{3}\right) 7.22-7.11(4 \mathrm{H}, \mathrm{m}), 5.75(1 \mathrm{H}, \mathrm{s}), 3.18(1 \mathrm{H}, \mathrm{tt}, J=12, J=4), 2.87(1 \mathrm{H}, \mathrm{d}, J=15), 2.67(1 \mathrm{H}$, $\mathrm{d}, J=14), 2.34-2.08(3 \mathrm{H}, \mathrm{m}), 1.89(3 \mathrm{H}, \mathrm{s}) 1.83-1.85-1.65(1 \mathrm{H}, \mathrm{m}) ;{ }^{13} \mathrm{C}-\mathrm{NMR} \delta:\left(80 \mathrm{MHz}, \mathrm{CD}_{3} \mathrm{COCD}_{3}\right)$ 203.5, 202.7, 150.3, 150.0, 141.7, 141.6, 131.8, 131.7, 128.3, 128.2, 128.1, 98.2, 97.8, 88.6, 48.2, 46.6, 46.1, 44.1, 39.9, 37.9, 37.7, 35.0, 10.7. HRMS found: $\mathrm{MH}^{+} 323.0798, \mathrm{C}_{15} \mathrm{H}_{16} \mathrm{ClN}_{2} \mathrm{O}_{4}$ requires 323.0799; $m / z: 323\left(80 \%, \mathrm{MH}^{+}\right)$. 
8-Acetyl-9-(2-chloro-phenyl)-3-methyl-4-nitro-1-oxa-2-aza-spiro[4.5]dec-2-en-7-one 5k: colourless solid (709 mg, 65\% yield), m.p. $142-3{ }^{\circ} \mathrm{C}$ (ethanol); $v_{\max }\left(\right.$ Film) $/ \mathrm{cm}^{-1}: 1725 \mathrm{~s}, 1570 \mathrm{~s} ;{ }^{1} \mathrm{H}-\mathrm{NMR} \delta$ : $(200$ $\left.\mathrm{MHz}, \mathrm{CD}_{3} \mathrm{COCD}_{3}\right)$ 7.23-7.06 (4H, m), $5.60(1 \mathrm{H}, \mathrm{s}), 4.42-4.32(1 \mathrm{H}, \mathrm{m}), 3.00(1 \mathrm{H}, \mathrm{d}, J=15), 2.29-2.12$ $(2 \mathrm{H}, \mathrm{m}), 1.90(3 \mathrm{H}, \mathrm{s}), 1.65-1.46(1 \mathrm{H}, \mathrm{m}) 1.43(3 \mathrm{H}, \mathrm{s}) ;{ }^{13} \mathrm{C}-\mathrm{NMR} \delta:\left(80 \mathrm{MHz}, \mathrm{CD}_{3} \mathrm{COCD}_{3}\right)$ 198.9, 178.1, $150.1,142.2,132.1,129.3,128.4,127.9,127.8,109.0,97.6,85.1,38.2,35.0,24.6,17.5,10.7$; HRMS found: $\mathrm{MH}^{+} 365.0905, \mathrm{C}_{17} \mathrm{H}_{18} \mathrm{ClN}_{2} \mathrm{O}_{5}$ requires $365.0904 ; m / z: 365\left(100 \%, \mathrm{MH}^{+}\right)$.

7-(2-Chloro-phenyl)-3-methyl-4-nitro-9-oxo-1-oxa-2-aza-spiro[4.5]dec-2-ene-8-carboxylic acid ethyl ester 5l: colourless solid (697 mg, 59\% yield), m.p.131-2 ${ }^{\circ} \mathrm{C}$ (ethanol); $v_{\max }\left(\right.$ Film)/ $\mathrm{cm}^{-1}: 1731 \mathrm{~s}$, 1570s; ${ }^{1} \mathrm{H}-\mathrm{NMR} \delta:\left(200 \mathrm{MHz}, \mathrm{CDCl}_{3}\right)$ 7.40-7.18 (4H, m), $5.35(1 \mathrm{H}, \mathrm{s}), 4.60-4.23(1 \mathrm{H}, \mathrm{m}), 4.20-3.82$ (3H, m), $2.83(1 \mathrm{H}, \mathrm{d}, J=14), 2.73(1 \mathrm{H}, \mathrm{dd}, J=14, J=2), 2.39-2.12(2 \mathrm{H}, \mathrm{m}), 2.14(3 \mathrm{H}, \mathrm{s}), 1.06(3 \mathrm{H}, \mathrm{t}, J$ = 7)); ${ }^{13} \mathrm{C}-\mathrm{NMR} \delta:\left(80 \mathrm{MHz}, \mathrm{CDCl}_{3}\right) 199.1,167.1,150.2,150.1,139.4,132.4,129.3,128.2,128.1$, $127.5,127.3,127.1,127.0,97.9,96.7,88.4,88.3,61.8,61.5,59.2,48.2,43.2,41.9,41.5,40.1,35.6$, 13.1, 10.7; HRMS found: $\mathrm{MH}^{+} 395.1002, \mathrm{C}_{18} \mathrm{H}_{20} \mathrm{ClN}_{2} \mathrm{O}_{6}$ requires 395.1001; $\mathrm{m} / z: 395\left(100 \%, \mathrm{MH}^{+}\right)$.

9-(2-Chloro-phenyl)-3-methyl-4-nitro-1-oxa-2-aza-spiro[4.5]dec-2-en-7-one $5 \mathrm{~m}$ : colourless solid (656 mg, 68\% yield), m.p. $125-6{ }^{\circ} \mathrm{C}$ (ethanol), $v_{\max }\left(\right.$ Film)/ $\mathrm{cm}^{-1}: 1702 \mathrm{~s}, 1570 \mathrm{~s} ;{ }^{1} \mathrm{H}-\mathrm{NMR} \delta:(200 \mathrm{MHz}$, $\left.\mathrm{CDCl}_{3}\right) 7.32(1 \mathrm{H}, \mathrm{d}, J=7), 7.23-7.05(3 \mathrm{H}, \mathrm{m}), 5.76(1 \mathrm{H}, \mathrm{s}), 3.70(1 \mathrm{H}, \mathrm{tt}, J=12, J=4), 2.90(1 \mathrm{H}, \mathrm{d}, J=$ 14), $2.72(1 \mathrm{H}, \mathrm{t}, J=14) 2.40-2.06(3 \mathrm{H}, \mathrm{m}), 1.90(3 \mathrm{H}, \mathrm{s}), 1.89-1.65(1 \mathrm{H}, \mathrm{m}) ;{ }^{13} \mathrm{C}-\mathrm{NMR} \delta:(80 \mathrm{MHz}$, $\left.\mathrm{CD}_{3} \mathrm{COCD}_{3}\right)$ 202.8, 202.7, 150.2, 150.1, 139.5, 132.6, 129.3, 128.1, 128.0, 127.3, 127.2, 127.1, 127.0, 97.9, 96.7, 88.5, 88.4, 48.4, 45.0, 44.6, 43.5, 38.6, 34.3, 34.2, 33.7, 10.8. HRMS found: $\mathrm{MH}^{+}$323.0798, $\mathrm{C}_{15} \mathrm{H}_{16} \mathrm{ClN}_{2} \mathrm{O}_{4}$ requires 323.0799; $m / z: 323\left(80 \%, \mathrm{MH}^{+}\right)$. 
NMR $\delta:\left(200 \mathrm{MHz}, \mathrm{CDCl}_{3}\right) 7.10(2 \mathrm{H}, \mathrm{d}, J=8), 6.80(2 \mathrm{H}, \mathrm{d}, J=8), 5.41(1 \mathrm{H}, \mathrm{s}), 3.80(1 \mathrm{H}, \mathrm{d}, J=15)$,

3.75-3.68 (4H, m), $2.74(2 \mathrm{H}, \mathrm{m}), 2.28-2.05(2 \mathrm{H}, \mathrm{m}), 2.12(3 \mathrm{H}, \mathrm{s}), 2.00(3 \mathrm{H}, \mathrm{s}) ;{ }^{13} \mathrm{C}-\mathrm{NMR} \delta:(80 \mathrm{MHz}$, $\left.\mathrm{CDCl}_{3}\right)$ 202.9, 202.4, 202.2, 158.5, 158.4, 150.4, 150.2, 132.9, 132.8, 128.0, 127.9, 113.7, 113.6, 97.8, 97.4, 88.1, 66.8, 66.4, 54.1, 48.5, 44.6, 40.8, 40.4, 40.3, 36.0, 29.9, 10.7. HRMS found: $\mathrm{MH}^{+}$361.1400, $\mathrm{C}_{18} \mathrm{H}_{21} \mathrm{~N}_{2} \mathrm{O}_{6}$ requires $361.1399 ; m / z: 361\left(100 \%, \mathrm{MH}^{+}\right)$.

7-(4-Methoxy-phenyl)-3-methyl-4-nitro-9-oxo-1-oxa-2-aza-spiro[4.5]dec-2-ene-8-carboxylic acid ethyl ester 5o: colourless solid (842 mg, $72 \%$ yield), m.p. $151-2{ }^{\circ} \mathrm{C}$ (ethanol); $v_{\max }($ Film $) / \mathrm{cm}^{-1}: 1722 \mathrm{~s}$, 1572s; ${ }^{1} \mathrm{H}-\mathrm{NMR} \delta:\left(200 \mathrm{MHz}, \mathrm{CDCl}_{3}\right) 7.15(2 \mathrm{H}, \mathrm{d}, J=8), 6.85(2 \mathrm{H}, \mathrm{d}, J=8), 5.35(1 \mathrm{H}, \mathrm{s}), 4.20-3.98$ $(2 \mathrm{H}, \mathrm{m}), 3.90-3.73(1 \mathrm{H}, \mathrm{m}), 3.78(3 \mathrm{H}, \mathrm{s}), 3.65(1 \mathrm{H}, \mathrm{d}, J=13), 2.79(1 \mathrm{H}, \mathrm{d}, J=15), 2.69(1 \mathrm{H}, \mathrm{d}, J=15)$, 2.28-2.07 (2H, m), $2.15(3 \mathrm{H}, \mathrm{s}), 1.08(3 \mathrm{H}, \mathrm{t}, J=7) ;{ }^{13} \mathrm{C}-\mathrm{NMR} \delta:\left(80 \mathrm{MHz}, \mathrm{CDCl}_{3}\right)$ 197.9, 166.5, 157.9, $148.4,131.0,127.2,127.1,113.3,97.5,97.3,87.2,62.0,61.8,60.0,54.2,48.0,44.4,40.4,39.9,39.7$, 35.1, 12.9, 10.7. HRMS found: $\mathrm{MH}^{+} 391.1506, \mathrm{C}_{19} \mathrm{H}_{23} \mathrm{~N}_{2} \mathrm{O}_{7}$ requires 391.1505; m/z: $391\left(100 \%, \mathrm{MH}^{+}\right)$.

9-(4-Methoxy-phenyl)-3-methyl-4-nitro-1-oxa-2-aza-spiro[4.5]dec-2-en-7-one 5p: colourless solid (735 mg, 77\% yield), m.p. $146-7{ }^{\circ} \mathrm{C}$ (ethanol); $v_{\max }\left(\right.$ Film)/cm ${ }^{-1}: 1709 \mathrm{~s}, 1570 \mathrm{~s} ;{ }^{1} \mathrm{H}-\mathrm{NMR} \delta:(200 \mathrm{MHz}$, $\left.\mathrm{CDCl}_{3}\right) 7.15(2 \mathrm{H}, \mathrm{d}, J=8), 6.88(2 \mathrm{H}, \mathrm{d}, J=8), 5.38(1 \mathrm{H}, \mathrm{s}), 3.80(3 \mathrm{H}, \mathrm{s}), 3.46(1 \mathrm{H}, \mathrm{tt}, J=12, J=4)$, 2.76-2.47 (4H, m), 2.25-2.01 (2H, m), $2.13(3 \mathrm{H}, \mathrm{s}) ;{ }^{13} \mathrm{C}-\mathrm{NMR} \delta:\left(80 \mathrm{MHz}, \mathrm{CDCl}_{3}\right)$ 203.4, 202.7, 158.3, $150.7,150.2,134.7,134.4,127.4,127.3,113.7,113.6,98.0,97.7,88.7,54.1,48.2,47.1,46.6,43.4$, 40.4, 37.8, 37.6, 35.6, 10.8. HRMS found: $\mathrm{MH}^{+} 319.1295, \mathrm{C}_{16} \mathrm{H}_{19} \mathrm{~N}_{2} \mathrm{O}_{5}$ requires 319.1294; m/z: 319 $\left(90 \%, \mathrm{MH}^{+}\right)$.

3-[2-(3-Methyl-4-nitro-isoxazol-5-yl)-1-(4-nitro-phenyl)-ethyl]-pentane-2,4-dione $3 \mathrm{~d}$ : colourless solid (920 mg, $82 \%$ yield), $\mathrm{R}_{\mathrm{f}}=0.2$ (ethyl acetate : petroleum ether 1:3), m.p. $131-2{ }^{\circ} \mathrm{C}$ (ethanol); $v_{\max }$ (Film)/cm ${ }^{-1}: 1710 \mathrm{~s}, 1600 \mathrm{~s}, 1560 \mathrm{~m} ;{ }^{1} \mathrm{H}-\mathrm{NMR} \delta:\left(400 \mathrm{MHz}, \mathrm{CDCl}_{3}\right) 8.15(2 \mathrm{H}, J=8), 7.37(2 \mathrm{H}, J=8)$, 
4.35-4.28 (2H, m), $3.52(1 \mathrm{H}, \mathrm{dd}, J=14, J=9), 3.35(1 \mathrm{H}, \mathrm{dd}, J=14, J=5), 2.49(3 \mathrm{H}, \mathrm{s}), 2.36(3 \mathrm{H}, \mathrm{s})$, $1.95(3 \mathrm{H}, \mathrm{s}) ;{ }^{13} \mathrm{C}-\mathrm{NMR} \delta:\left(100 \mathrm{MHz}, \mathrm{CDCl}_{3}\right)$ 200.7, 200.3, 170.4, 155.2, 147.0, 145.3, 130.9, 128.4, 123.8, 73.7, 41.7, 31.4, 29.3, 29.1, 11.5; HRMS found: $\mathrm{MH}^{+} 376.1146, \mathrm{C}_{17} \mathrm{H}_{18} \mathrm{~N}_{3} \mathrm{O}_{7}$ requires 376.1145; $m / z: 376\left(100 \%, \mathrm{MH}^{+}\right)$.

2-Acetyl-4-(3-methyl-4-nitro-isoxazol-5-yl)-3-(4-nitro-phenyl)-butyric acid ethyl ester 3e: colourless solid (875 mg, 74\% yield), $\mathrm{R}_{\mathrm{f}}=0.4$ (ethyl acetate : petroleum ether 1:3), m.p. $95-8{ }^{\circ} \mathrm{C}$ (ethanol); $v_{\max }\left(\right.$ Film)/cm ${ }^{-1}: 1725 \mathrm{~s}, 1600 \mathrm{~s}, 1560 \mathrm{~m} ;{ }^{1} \mathrm{H}-\mathrm{NMR} \delta:\left(200 \mathrm{MHz}, \mathrm{CDCl}_{3}\right) 8.01(2 \mathrm{H}, \mathrm{d}, J=8)$, $7.37(2 \mathrm{H}, \mathrm{d}, J=8), 4.31-3.81(4 \mathrm{H}, \mathrm{m}), 3.74-3.46(2 \mathrm{H}, \mathrm{m}), 2.44(3 \mathrm{H}, \mathrm{s}), 2.34(1.5 \mathrm{H}, \mathrm{s}), 2.06(1.5 \mathrm{H}, \mathrm{s})$, $1.27(1.5 \mathrm{H}, \mathrm{t}, J=7), 0.98(1.5 \mathrm{H}, \mathrm{t}, J=7) ;{ }^{13} \mathrm{C}-\mathrm{NMR} \delta:\left(80 \mathrm{MHz}, \mathrm{CDCl}_{3}\right)$ 198.9, 198.6, 170.2, 166.0, $165.5,154.4,154.3,146.3,145.3,129.4,127.9,122.8,63.5,63.2,61.3,60.8,30.5,30.1,28.9,28.3$, 12.8, 12.5, 10.2; HRMS found: $\mathrm{MH}^{+} 406.1248, \mathrm{C}_{18} \mathrm{H}_{20} \mathrm{~N}_{3} \mathrm{O}_{8}$ requires 406.1250; $m / z: 406\left(100 \%, \mathrm{MH}^{+}\right)$.

5-(3-Methyl-4-nitro-isoxazol-5-yl)-4-(4-nitro-phenyl)-pentan-2-one 3f: colourless oil (798 mg, 80\% yield), $\mathrm{R}_{\mathrm{f}}=0.4$ (ethyl acetate : petroleum ether $\left.1: 4\right) ; v_{\max }\left(\right.$ Film) $/ \mathrm{cm}^{-1}: 1710 \mathrm{~s}, 1601 \mathrm{~s}, 1580 \mathrm{~s} ;{ }^{1} \mathrm{H}-\mathrm{NMR} \delta$ : $\left(400 \mathrm{MHz}, \mathrm{CDCl}_{3}\right) 8.17(2 \mathrm{H}, \mathrm{d}, J=8), 7.42(2 \mathrm{H}, \mathrm{d}, J=8), 4.03(1 \mathrm{H}$, quintet, $J=7), 3.66(1 \mathrm{H}, \mathrm{dd}, J=$ $14, J=7), 3.53(1 \mathrm{H}, \mathrm{dd}, J=14, J=7), 2.99(1 \mathrm{H}, \mathrm{dd}, J=14, J=4), 2.95(1 \mathrm{H}, \mathrm{dd}, J=14, J=4), 2.52$ $(3 \mathrm{H}, \mathrm{s}), 2.14(3 \mathrm{H}, \mathrm{s}) ;{ }^{13} \mathrm{C}-\mathrm{NMR} \delta:\left(100 \mathrm{MHz}, \mathrm{CDCl}_{3}\right)$ 204.4, 171.4, 155.2, 148.7, 146.2, 130.2, 127.8, 123.7, 48.2, 37.7, 32.8, 29.8, 11.1; HRMS found: $\mathrm{MH}^{+} 334.1037, \mathrm{C}_{15} \mathrm{H}_{16} \mathrm{~N}_{3} \mathrm{O}_{6}$ requires 334.1039; $m / z$ : $334\left(100 \%, \mathrm{MH}^{+}\right)$.

3-[1-(4-Chloro-phenyl)-2-(3-methyl-4-nitro-isoxazol-5-yl)-ethyl]-pentane-2,4-dione 3g: colourless solid (775 mg, $71 \%$ yield), $\mathrm{R}_{\mathrm{f}}=0.3$ (ethyl acetate : petroleum ether 1:4), m.p. $89-90{ }^{\circ} \mathrm{C}$ (ethanol); $v_{\max }$ $\left(\right.$ Film)/cm ${ }^{-1}: 1705 \mathrm{~s}, 1601 \mathrm{~s}, 1520 \mathrm{~m},{ }^{1} \mathrm{H}-\mathrm{NMR} \delta:\left(400 \mathrm{MHz}, \mathrm{CDCl}_{3}\right) 7.22(2 \mathrm{H}, J=8), 6.98(2 \mathrm{H}, J=8)$, $4.26(1 \mathrm{H}, \mathrm{d}, J=11), 4.18-4.12(1 \mathrm{H}, \mathrm{m}), 3.52(1 \mathrm{H}, \mathrm{dd}, J=14, J=9), 3.35(1 \mathrm{H}, \mathrm{dd}, J=14, J=5), 2.48$ 
$(3 \mathrm{H}, \mathrm{s}), 2.32(3 \mathrm{H}, \mathrm{s}), 1.90(3 \mathrm{H}, \mathrm{s}) ;{ }^{13} \mathrm{C}-\mathrm{NMR} \delta:\left(100 \mathrm{MHz}, \mathrm{CDCl}_{3}\right)$ 201.7, 201.3, 171.0, 155.0, 136.2, 133.0, 131.0, 129.3, 129.0, 74.1, 41.5, 31.7, 29.6, 29.1, 11.1; HRMS found: $\mathrm{MH}^{+}$365.0906, $\mathrm{C}_{17} \mathrm{H}_{18} \mathrm{ClN}_{2} \mathrm{O}_{5}$ requires 365.0904; $m / z$ : $365\left(100 \%, \mathrm{MH}^{+}\right)$.

2-Acetyl-3-(4-chloro-phenyl)-4-(3-methyl-4-nitro-isoxazol-5-yl)-butyric acid ethyl ester 3h: colourless solid (920 mg, 78\% yield), $\mathrm{R}_{\mathrm{f}}=0.3$ (ethyl acetate : petroleum ether 1:4), m.p. $94-5{ }^{\circ} \mathrm{C}$ (ethanol); IR: 1742s, 1601s, 1562s; ${ }^{1} \mathrm{H}-\mathrm{NMR} \delta:\left(200 \mathrm{MHz}, \mathrm{CDCl}_{3}\right) 7.21(2 \mathrm{H}, J=8), 7.10(2 \mathrm{H}, J=8)$, 4.09-3.94 (2H, m), $3.92(2 \mathrm{H}, \mathrm{q}, J=7), 3.62(1 \mathrm{H}, \mathrm{dd}, J=14, J=9), 3.45(1 \mathrm{H}, \mathrm{dd}, J=14, J=5), 2.46$ $(3 \mathrm{H}, \mathrm{s}), 2.32(3 \mathrm{H}, \mathrm{s}), 0.99(3 \mathrm{H}, \mathrm{t}, J=7) ;{ }^{13} \mathrm{C}-\mathrm{NMR} \delta:\left(80 \mathrm{MHz}, \mathrm{CDCl}_{3}\right)$ 199.6, 170.8, 165.5, 154.4, 136.0, 132.5, 130.5, 128.2, 127.8, 64.2, 60.7, 40.3, 30.8, 28.4, 16.6, 11.4; HRMS found: $\mathrm{MH}^{+} 395.1009$, $\mathrm{C}_{18} \mathrm{H}_{20} \mathrm{ClN}_{2} \mathrm{O}_{6}$ requires 395.1001; $m / z: 395\left(70 \%, \mathrm{MH}^{+}\right)$.

4-(4-Chloro-phenyl)-5-(3-methyl-4-nitro-isoxazol-5-yl)-pentan-2-one 3i: colourless oil (782 mg, $81 \%$ yield), $\mathrm{R}_{\mathrm{f}}=0.3$ (ethyl acetate : petroleum ether $\left.1: 5\right): v_{\max }\left(\right.$ Film) $/ \mathrm{cm}^{-1}: 1715 \mathrm{~s}, 1605 \mathrm{~s}, 1565 \mathrm{~s} ;{ }^{1} \mathrm{H}$ NMR $\delta:\left(400 \mathrm{MHz}, \mathrm{CDCl}_{3}\right) 7.25(2 \mathrm{H}, \mathrm{d}, J=8), 7.15(2 \mathrm{H}, \mathrm{d}, J=8), 3.82(1 \mathrm{H}$, quintet, $J=7), 3.57(1 \mathrm{H}$, $\mathrm{dd}, J=14, J=7), 3.44(1 \mathrm{H}, \mathrm{dd}, J=14, J=7), 2.89(2 \mathrm{H}$, app. septet, $J=17, J=7), 2.50(3 \mathrm{H}, \mathrm{s}), 2.10$ $(3 \mathrm{H}, \mathrm{s}) ;{ }^{13} \mathrm{C}-\mathrm{NMR} \delta:\left(100 \mathrm{MHz}, \mathrm{CDCl}_{3}\right)$ 205.1, 172.1, 155.1, 139.7, 132.6, 130.1, 128.55, 128.1, 48.6, 37.4, 33.2, 30.0, 11.2; HRMS found: $\mathrm{MH}^{+} 323.0801, \mathrm{C}_{15} \mathrm{H}_{16} \mathrm{ClN}_{2} \mathrm{O}_{4}$ requires 323.0799; m/z: 323 $\left(100 \%, \mathrm{MH}^{+}\right)$.

3-[1-(2-Chloro-phenyl)-2-(3-methyl-4-nitro-isoxazol-5-yl)-ethyl]-pentane-2,4-dione 3k: colourless solid (677 mg, 62\% yield), $\mathrm{R}_{\mathrm{f}}=0.3$ (ethyl acetate : petroleum ether 1:3), m.p. $85-6{ }^{\circ} \mathrm{C}$ (ethanol), $v_{\max }$ (Film)/cm ${ }^{-1}: 1705 \mathrm{~s}, 1605 \mathrm{~s}, 1535 \mathrm{~m} ;{ }^{1} \mathrm{H}-\mathrm{NMR} \delta:\left(400 \mathrm{MHz}, \mathrm{CDCl}_{3}\right)$ 7.34-7.19 (4H, m), 4.85-4.60 (1H, m), 4.45-4.39 (1H, m), 3.59-3.48 (2H, m), $2.48(3 \mathrm{H}, \mathrm{s}), 2.33(3 \mathrm{H}, \mathrm{s}), 1.94(3 \mathrm{H}, \mathrm{s}) ;{ }^{13} \mathrm{C}-\mathrm{NMR} \delta$ : $\left(100 \mathrm{MHz}, \mathrm{CDCl}_{3}\right)$ 202.1, 201.7, 172.1, 155.4, 138.4, 133.2, 130.6, 129.8, 128.5, 127.1, 126.5, 75.1, 
42.6, 32.0, 29.5, 29.3, 11.4; HRMS found: $\mathrm{MH}^{+}$365.0905, $\mathrm{C}_{17} \mathrm{H}_{18} \mathrm{ClN}_{2} \mathrm{O}_{5}$ requires 365.0904; m/z: 365 $\left(100 \%, \mathrm{MH}^{+}\right)$.

2-Acetyl-3-(2-chloro-phenyl)-4-(3-methyl-4-nitro-isoxazol-5-yl)-butyric acid ethyl ester 3l: colourless solid (614 mg, 52\% yield), $\mathrm{R}_{\mathrm{f}}=0.3$ (ethyl acetate : petroleum ether 1:3), m.p. $64-5{ }^{\circ} \mathrm{C}$ (ethanol), $v_{\max }\left(\right.$ Film)/cm ${ }^{-1}: 1720,1601,1525 \mathrm{~m} ;{ }^{1} \mathrm{H}-\mathrm{NMR} \delta:\left(200 \mathrm{MHz}, \mathrm{CDCl}_{3}\right)$ 7.32-7.15 (5H, m), 4.73-4.52 (1H, m), 4.32-4.09 (2H, m), $3.92(1 \mathrm{H}, \mathrm{q}, J=7), 3.80-3.54(2 \mathrm{H}, \mathrm{m}), 2.46(3 \mathrm{H}, \mathrm{s}), 2.35(1.6 \mathrm{H}$, s), $2.11(1.4 \mathrm{H}, \mathrm{s}), 1.30(1.4 \mathrm{H}, \mathrm{t}, J=7), 0.96(1.6 \mathrm{H}, \mathrm{t}, J=7) ;{ }^{13} \mathrm{C}-\mathrm{NMR} \delta:\left(80 \mathrm{MHz}, \mathrm{CDCl}_{3}\right)$ 199.5, 199.2, $170.7,166.6,165.7,154.2,135.2,134.8,133.1,129.5,130.0,127.9,127.8,126.2,125.9,62.7,62.2$, $60.9,60.6,29.5,29.1,28.3,28.0,12.9,12.4,10.3$; HRMS found: $\mathrm{MH}^{+} 395.1009, \mathrm{C}_{18} \mathrm{H}_{20} \mathrm{ClN}_{2} \mathrm{O}_{6}$ requires $395.1001 ; m / z: 395\left(100 \%, \mathrm{MH}^{+}\right)$.

4-(2-Chloro-phenyl)-5-(3-methyl-4-nitro-isoxazol-5-yl)-pentan-2-one 3m: colourless oil (695 mg, $72 \%$ yield), $\mathrm{R}_{\mathrm{f}}=0.3$ (ethyl acetate : petroleum ether $\left.1: 4\right), v_{\max }\left(\right.$ Film) $/ \mathrm{cm}^{-1}: 1715 \mathrm{~s}, 1600 \mathrm{~s}, 1565 \mathrm{~s} ;{ }^{1} \mathrm{H}-$ NMR $\delta:\left(400 \mathrm{MHz}, \mathrm{CDCl}_{3}\right) 7.34(1 \mathrm{H}, \mathrm{d}, J=8), 7.25-7.15(3 \mathrm{H}, \mathrm{m}), 4.38(1 \mathrm{H}$, quintet, $J=7), 3.60(2 \mathrm{H}$, d, $J=7), 2.96(2 \mathrm{H}, \mathrm{d}, J=6), 2.51(3 \mathrm{H}, \mathrm{s}), 2.15(3 \mathrm{H}, \mathrm{s}) ;{ }^{13} \mathrm{C}-\mathrm{NMR} \delta:\left(100 \mathrm{MHz}, \mathrm{CDCl}_{3}\right)$ 205.2, 172.0, 155.0, 138.2, 133.2, 130.4, 129.7, 128.0, 127.1, 126.8, 47.4, 34.9, 32.0, 30.0, 11.6; HRMS found: $\mathrm{MH}^{+}$ 323.0797, $\mathrm{C}_{15} \mathrm{H}_{16} \mathrm{ClN}_{2} \mathrm{O}_{4}$ requires 323.0798; $m / z: 323\left(100 \%, \mathrm{MH}^{+}\right)$. 
8-Acetyl-3-methyl-4-nitro-9-phenyl-1-oxa-2-aza-spiro[4.5]dec-2-en-7-one 5a

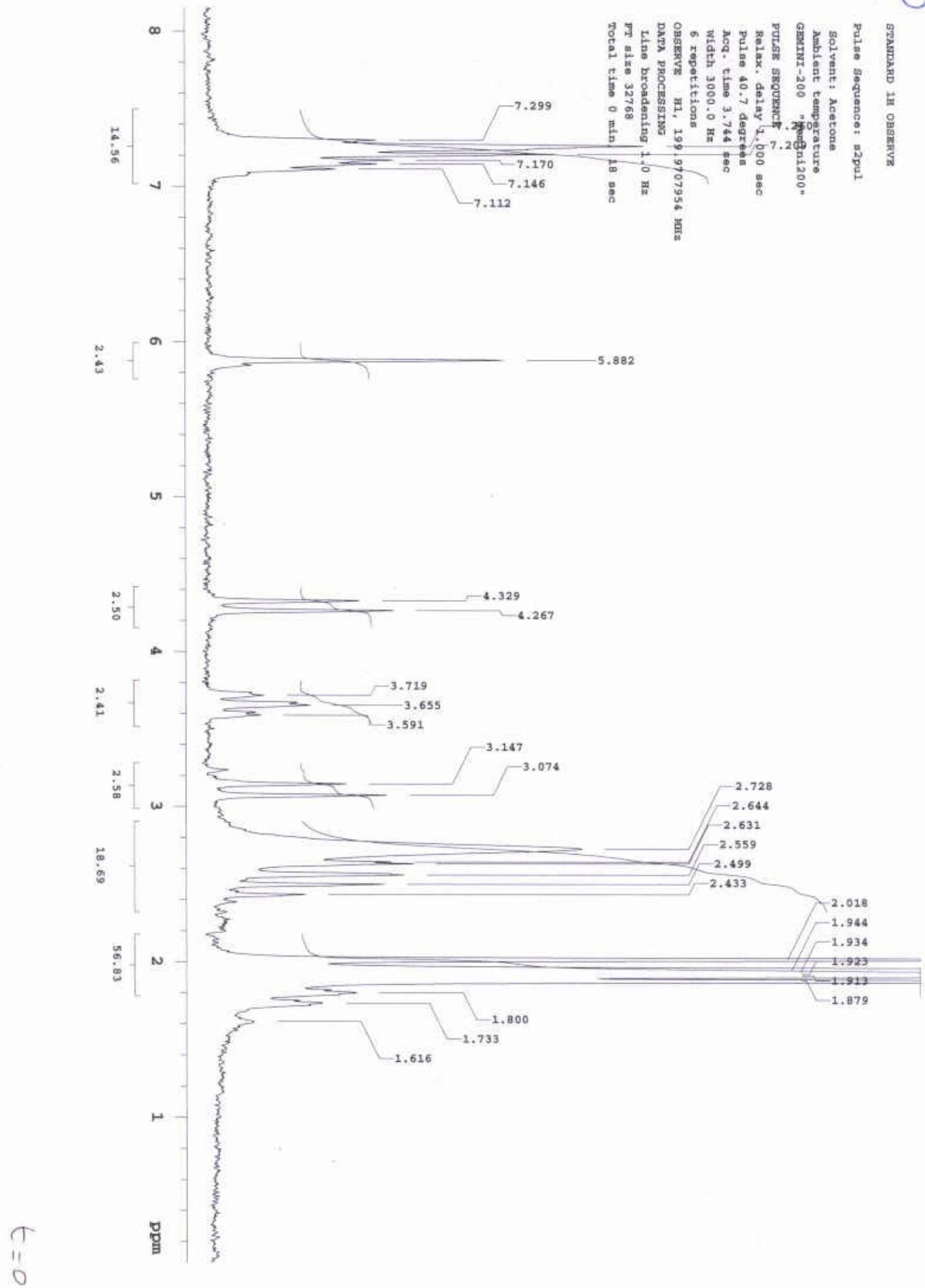




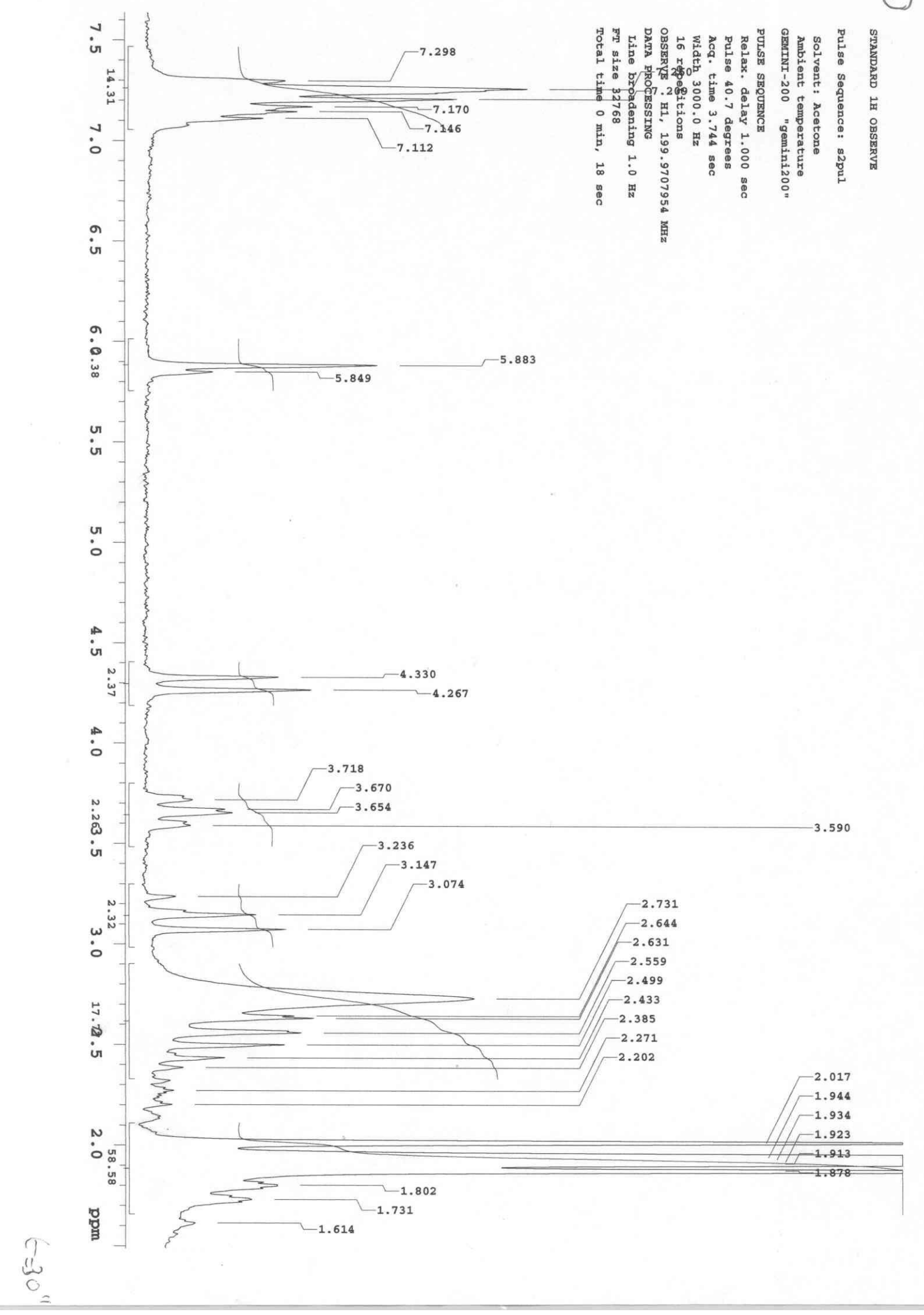




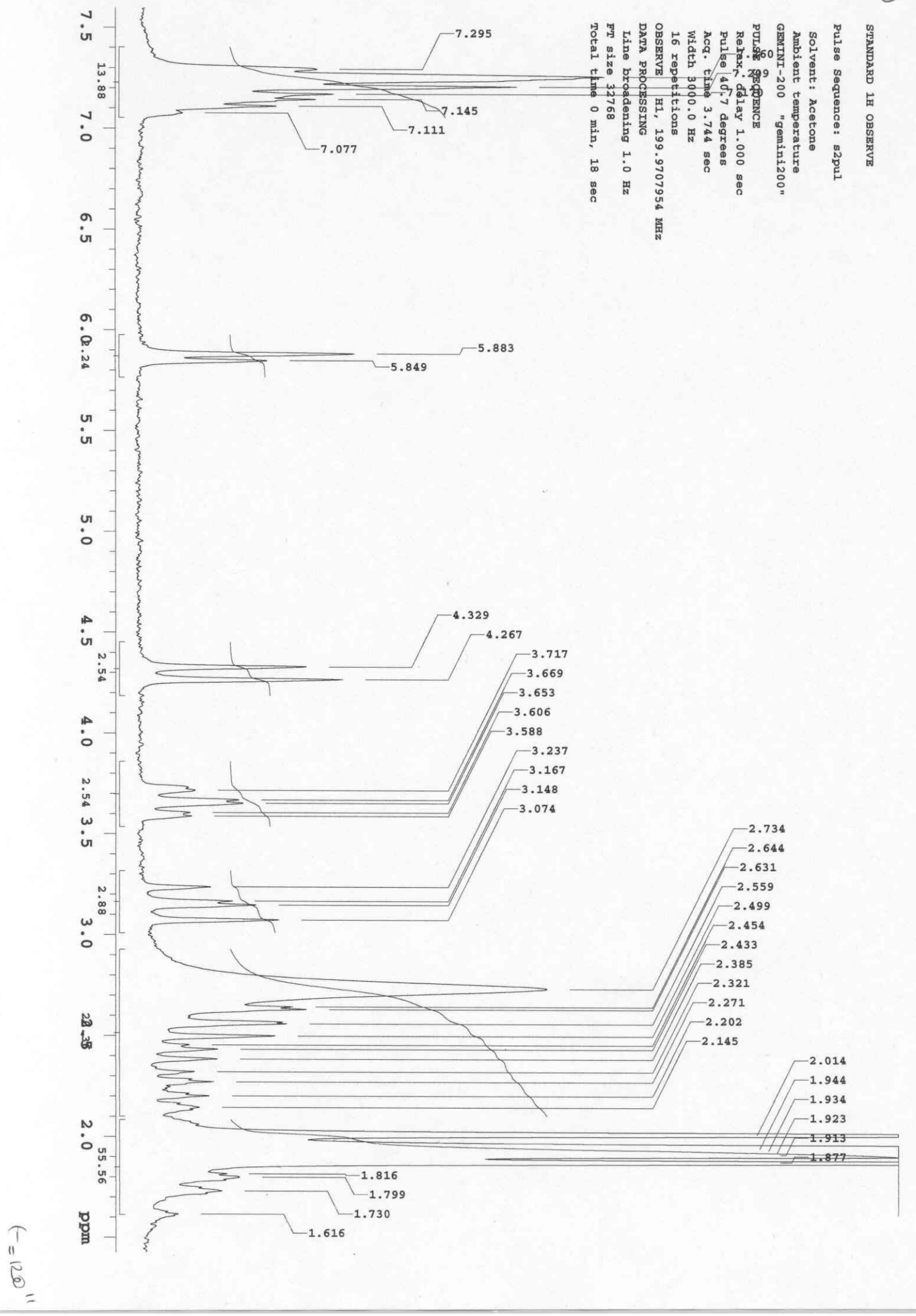


$\theta$

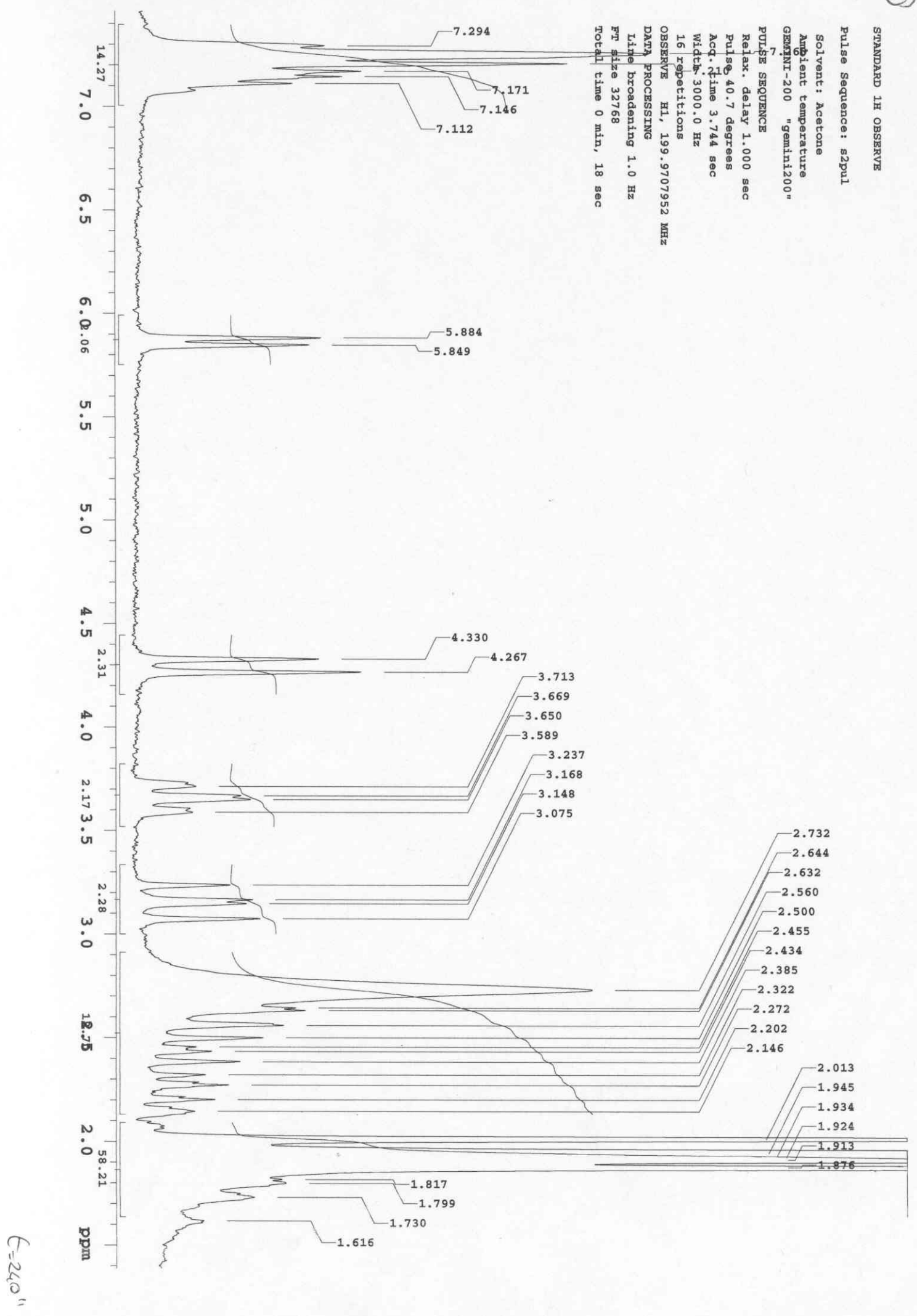




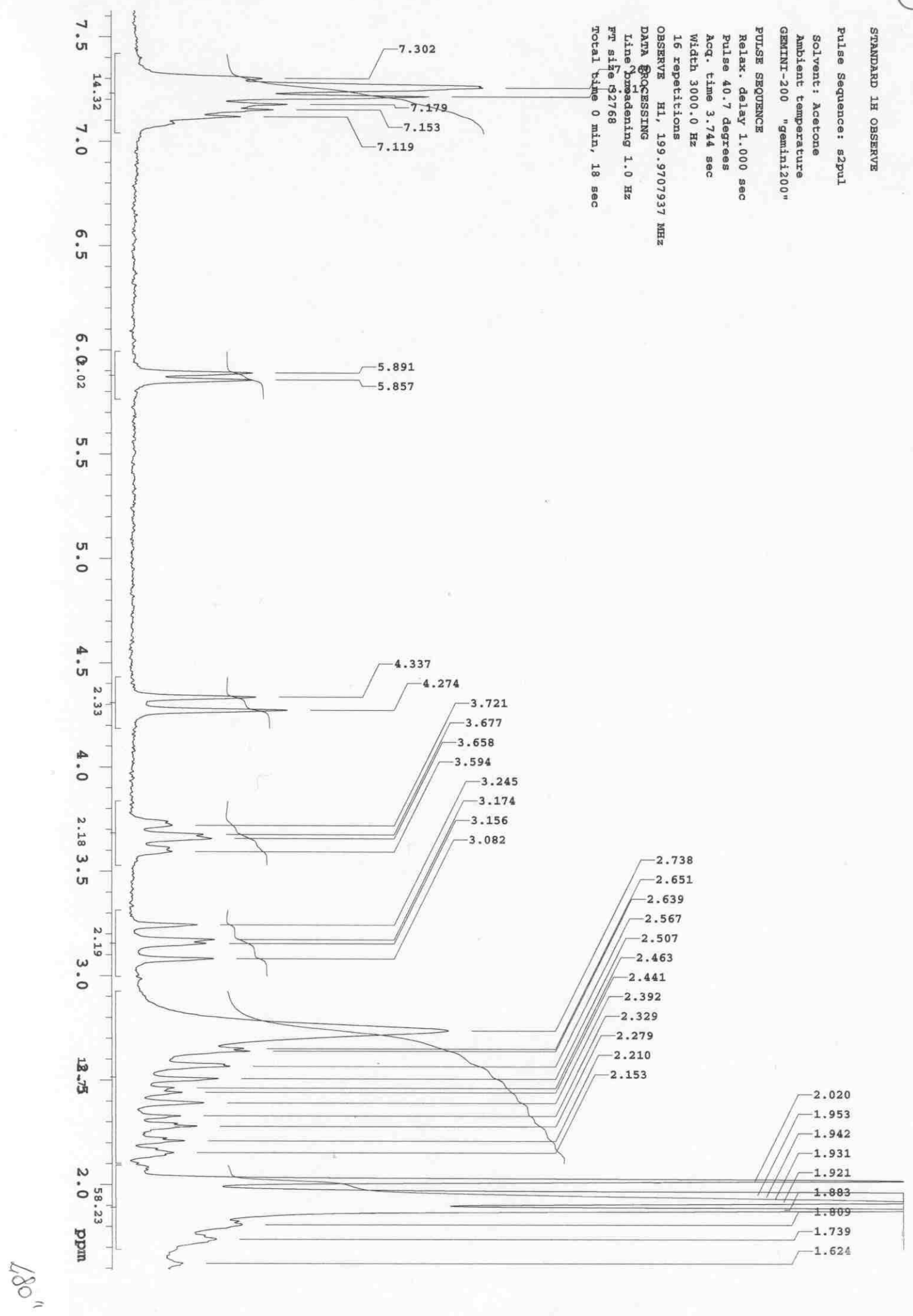


으

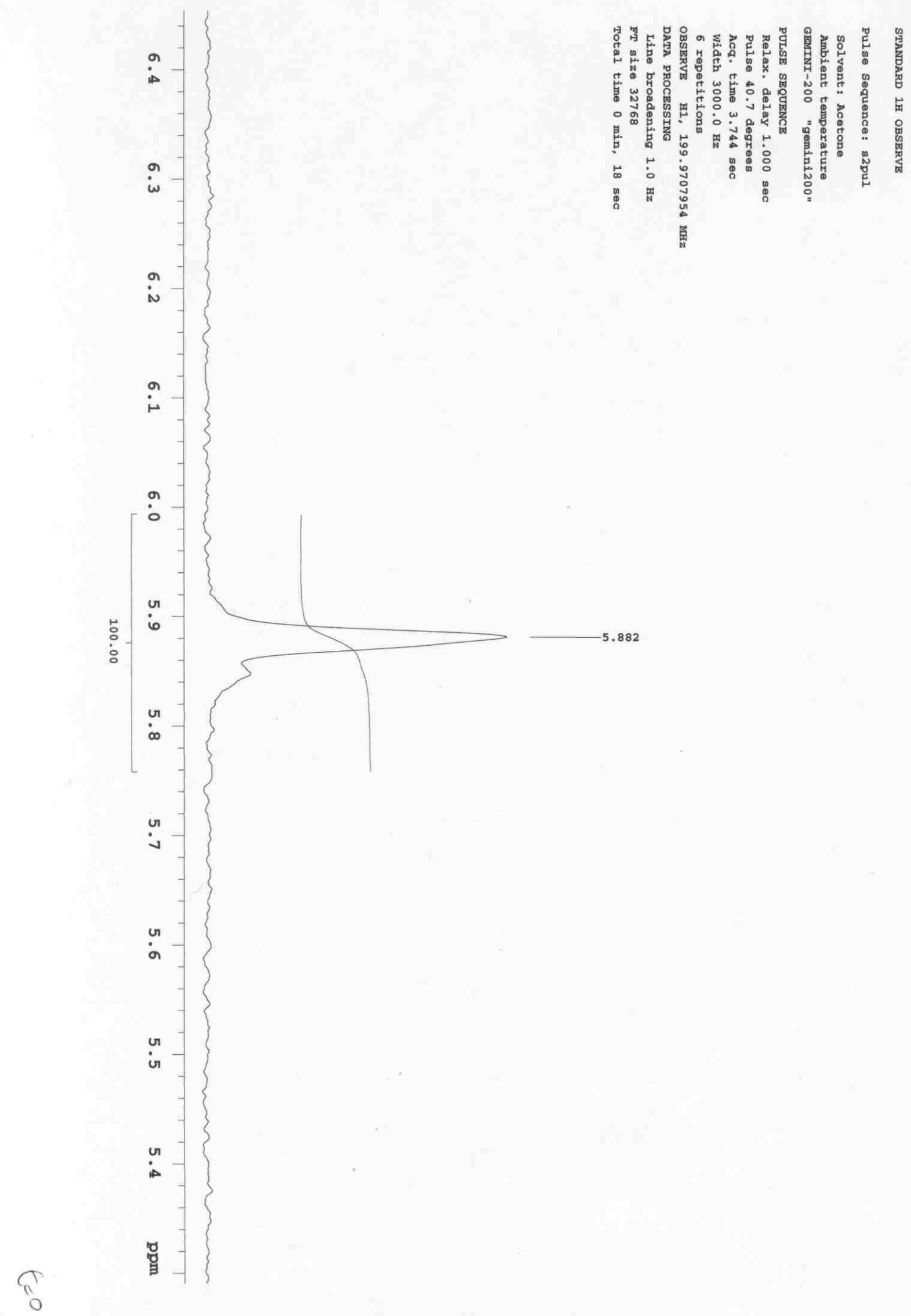


(

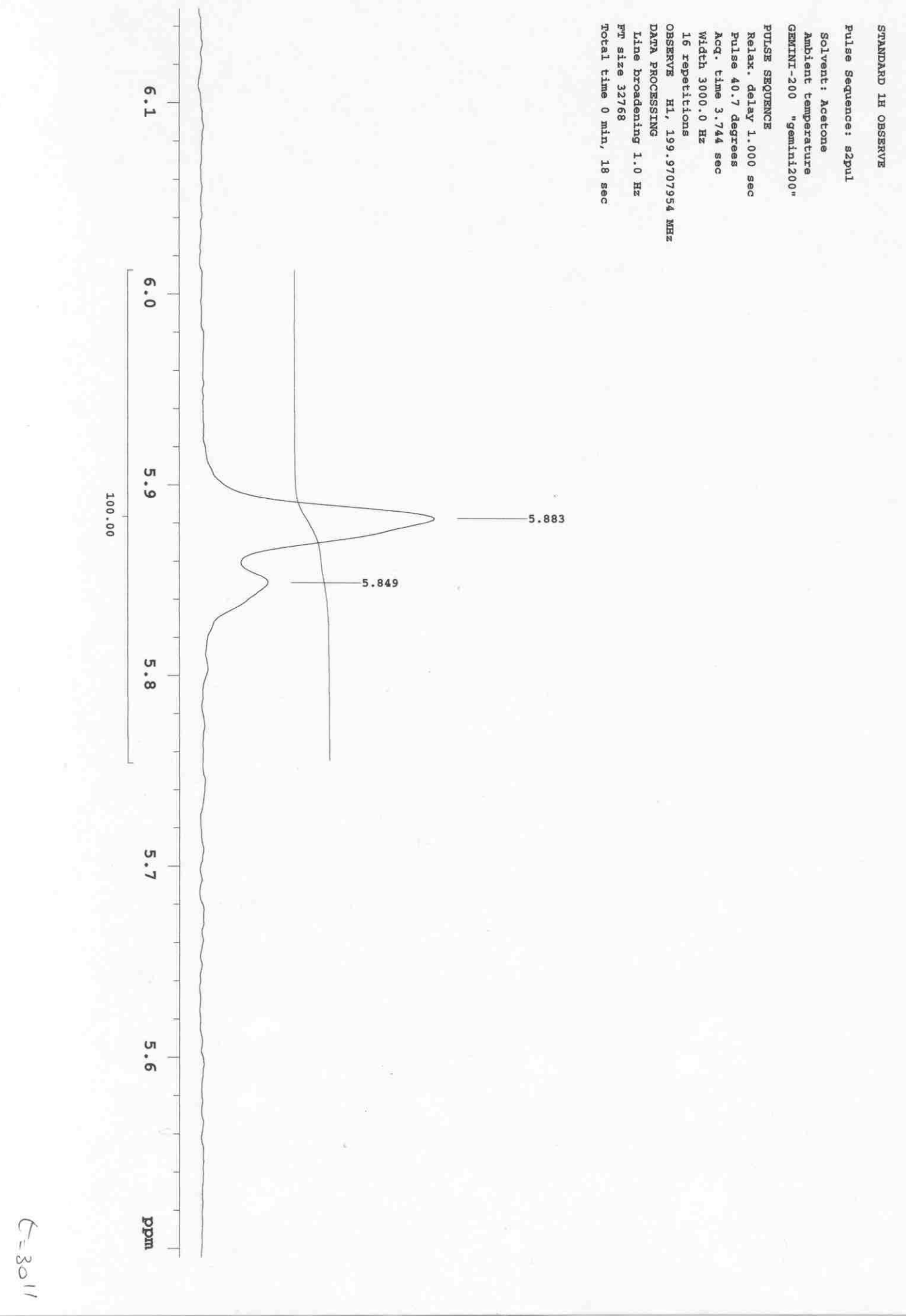


(D)

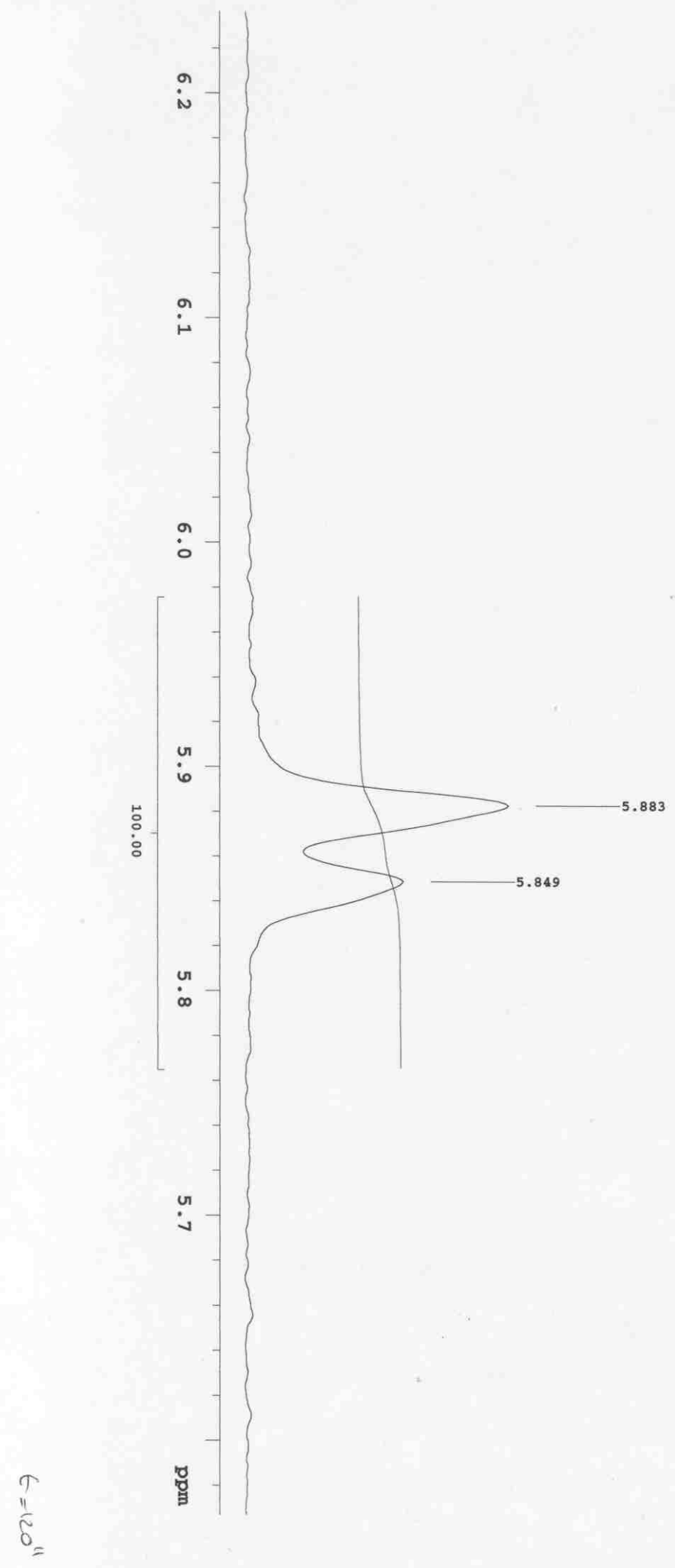



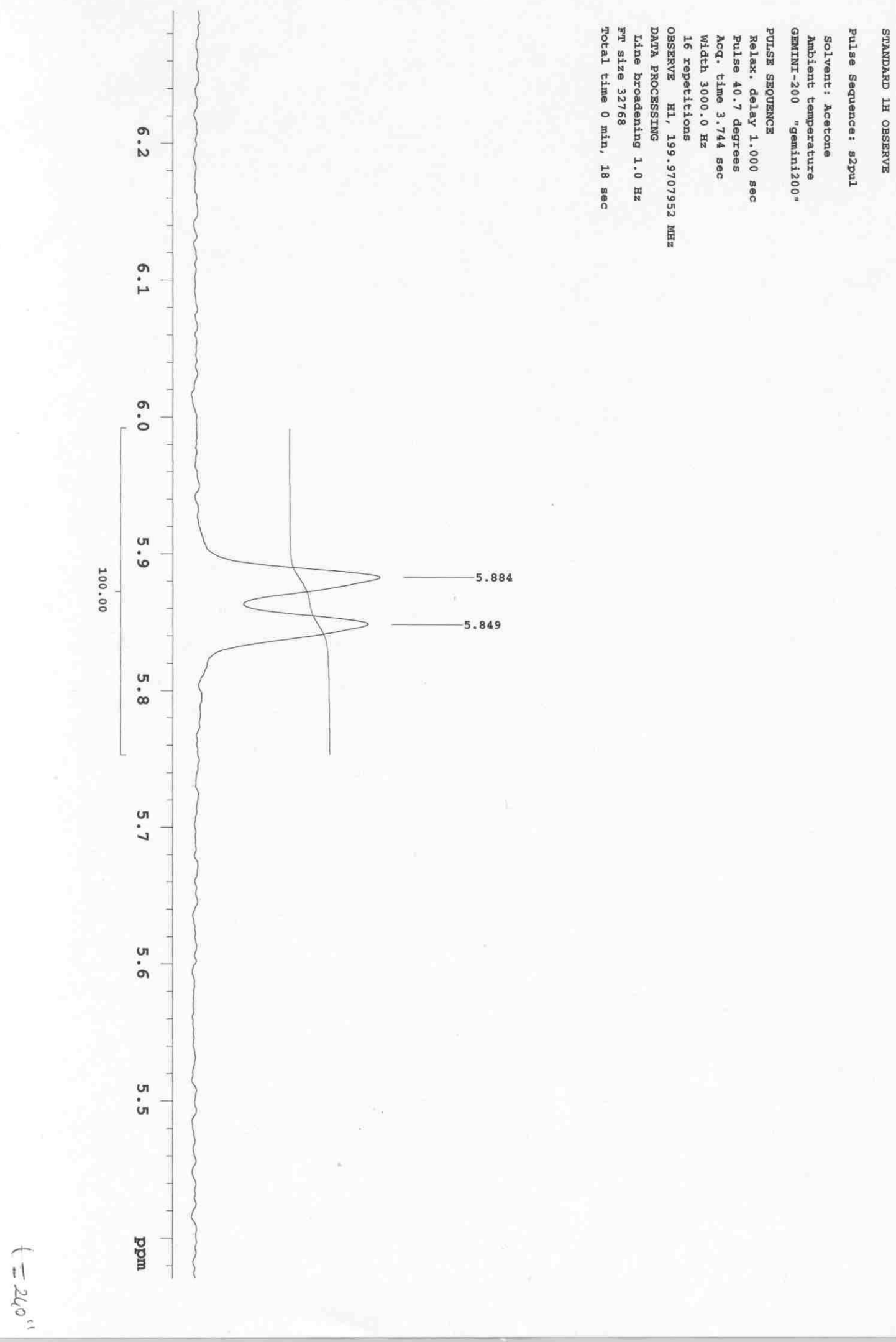


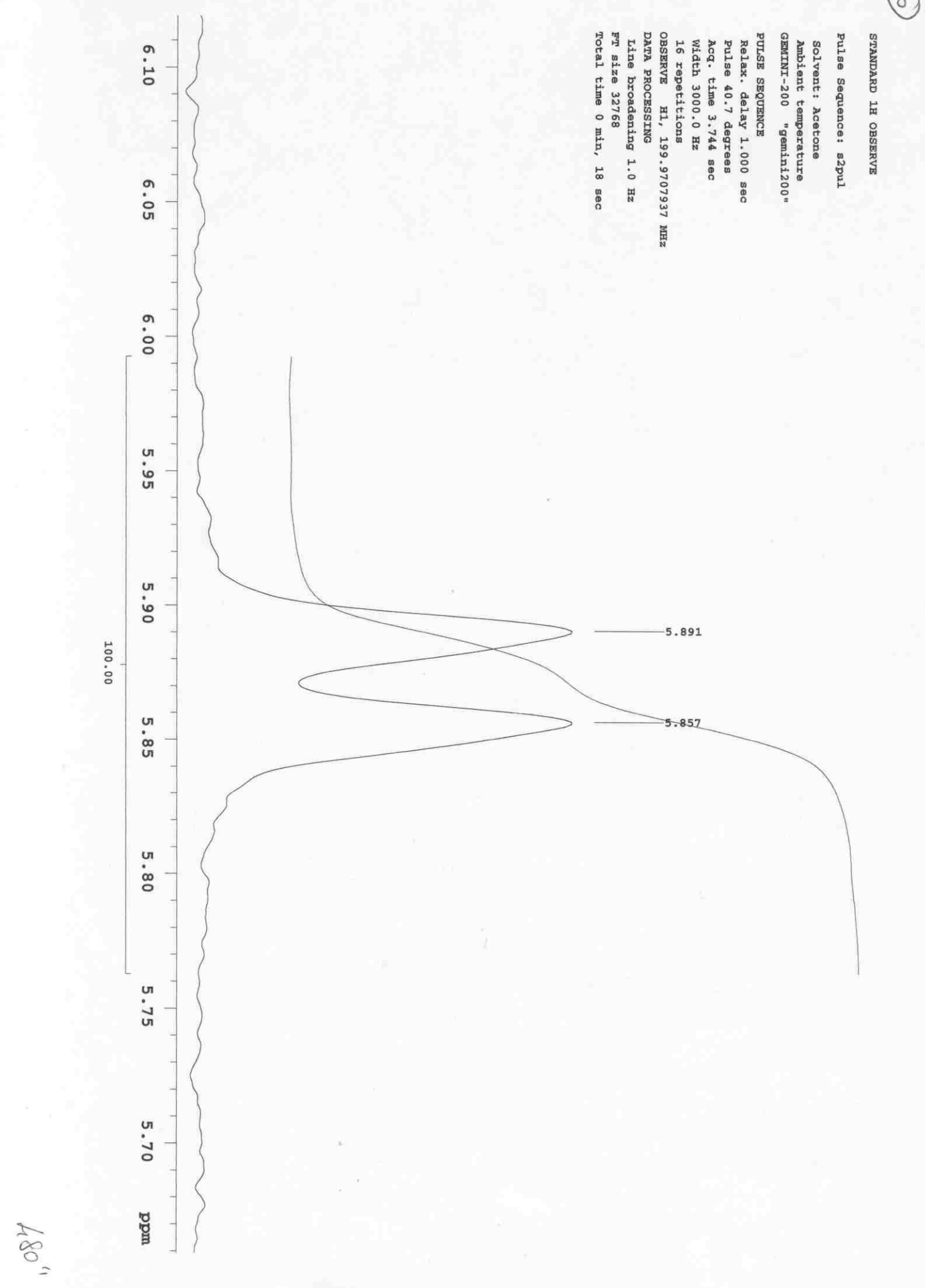


$\ominus$

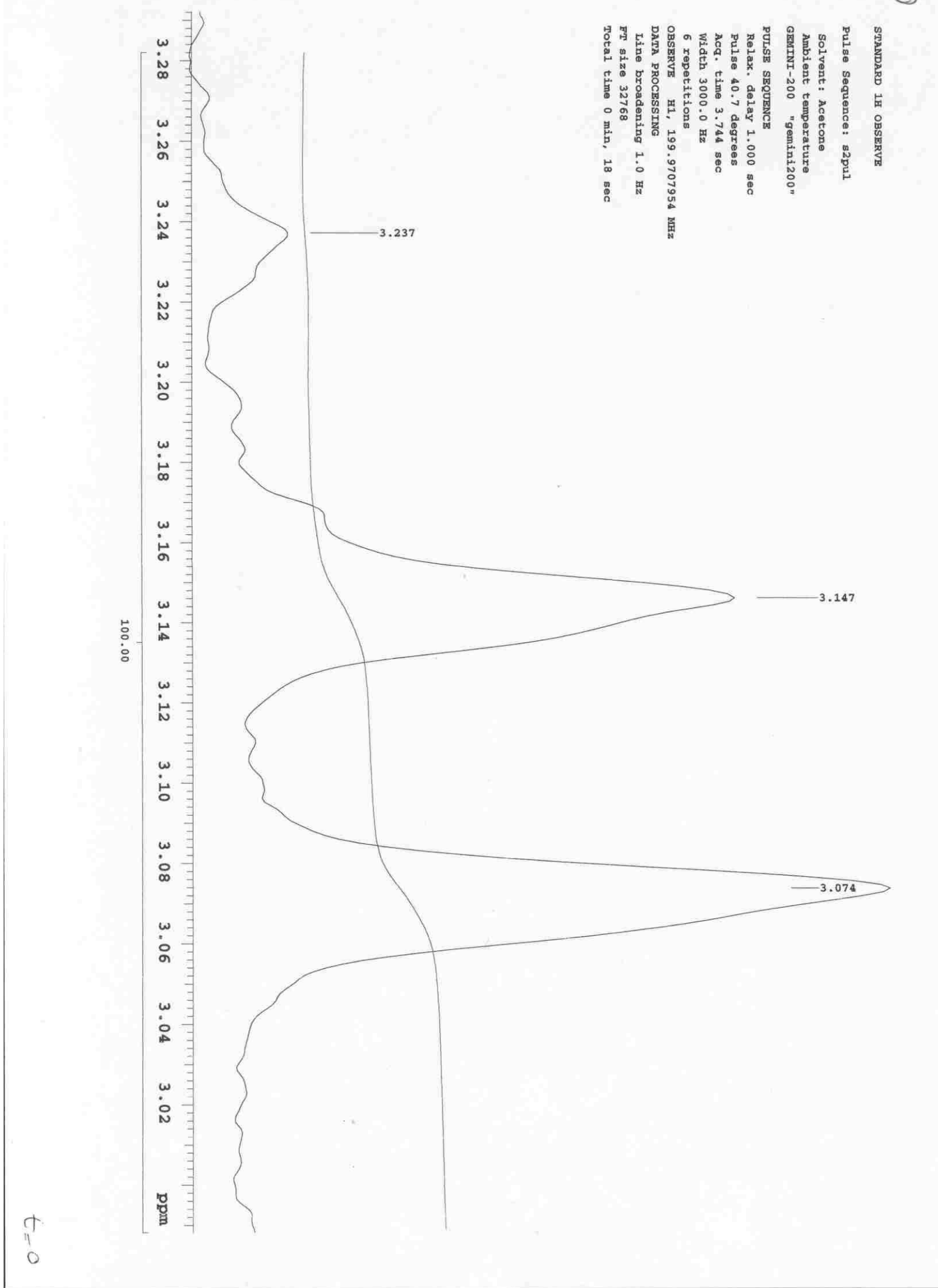




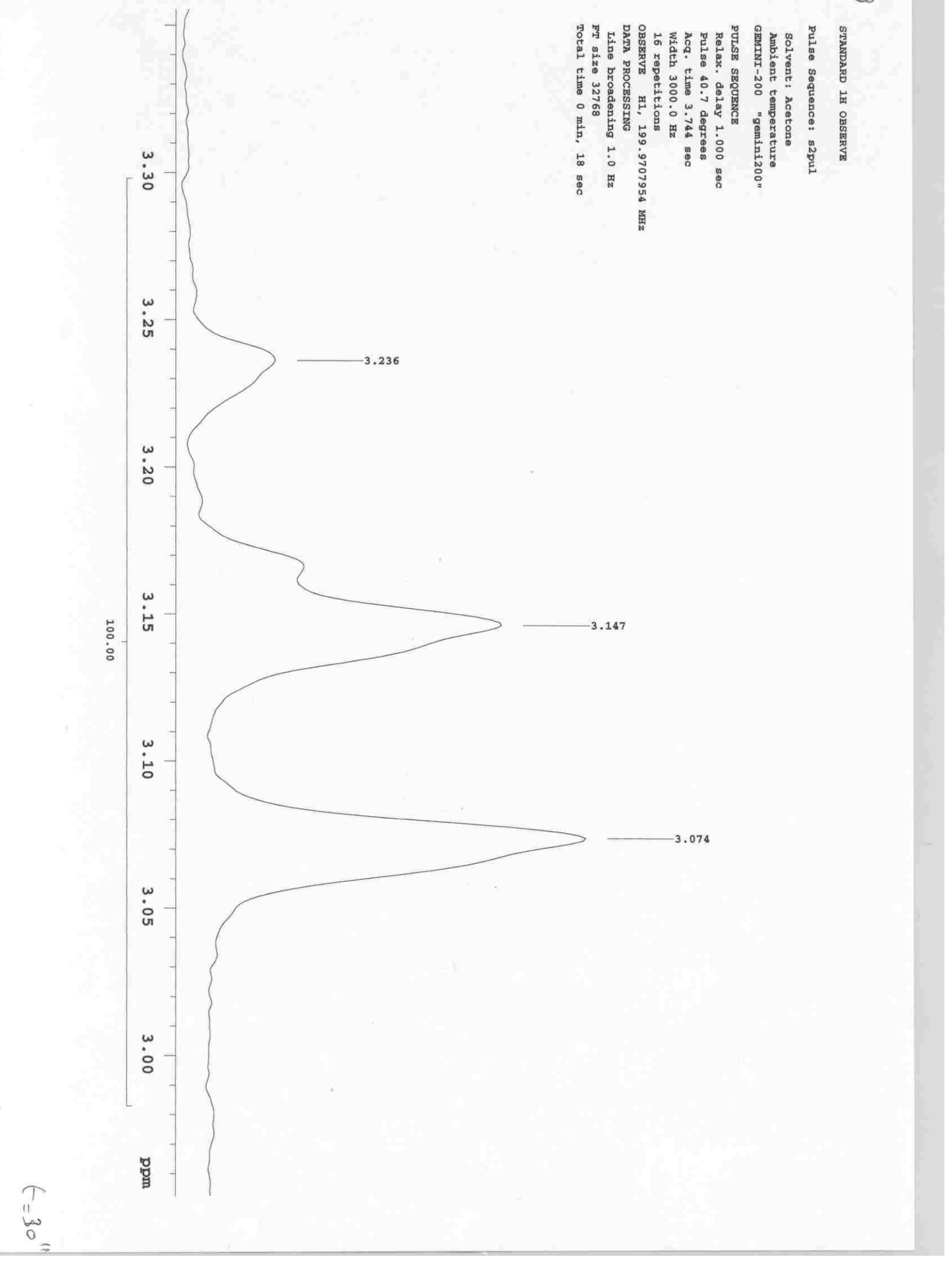




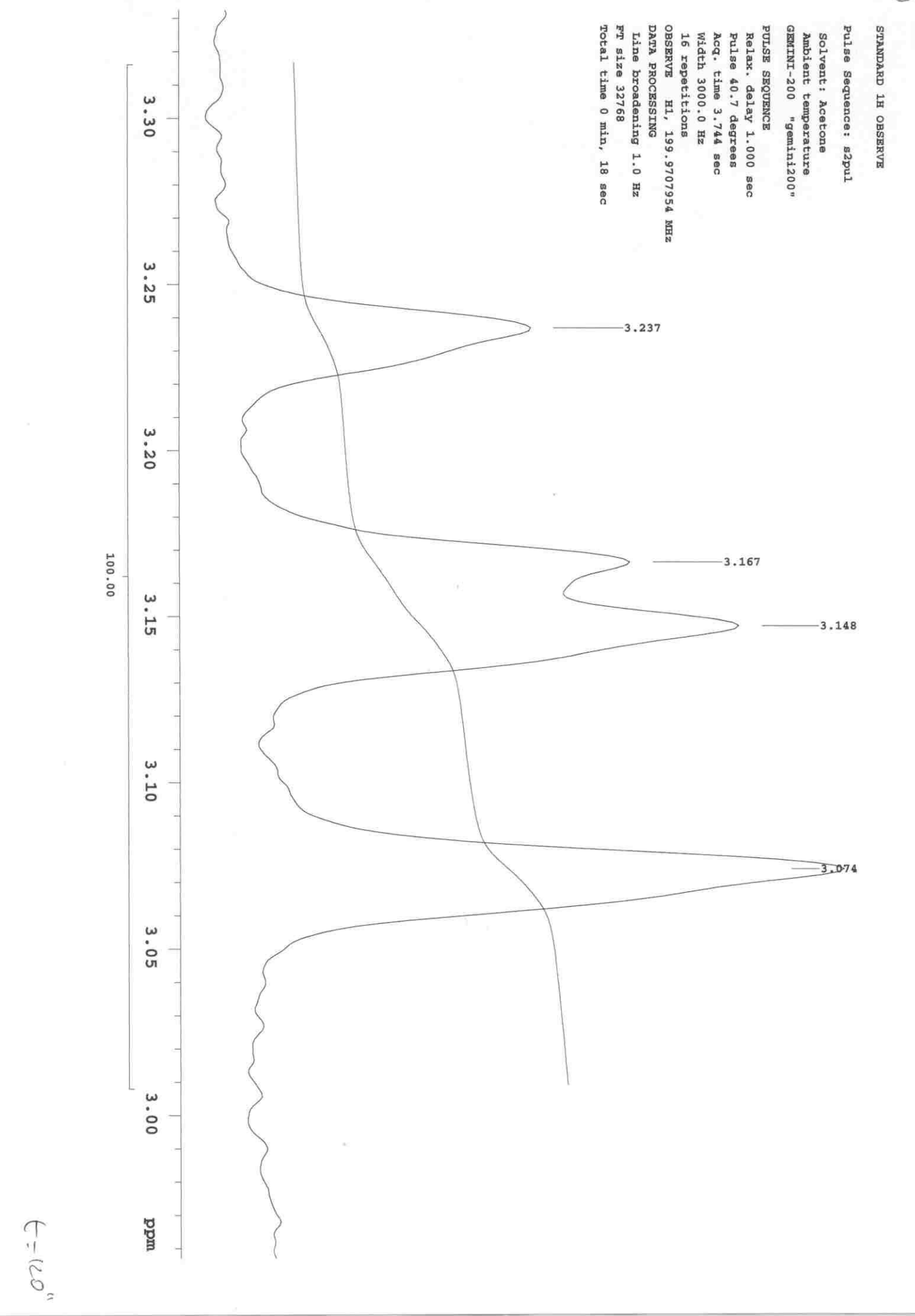




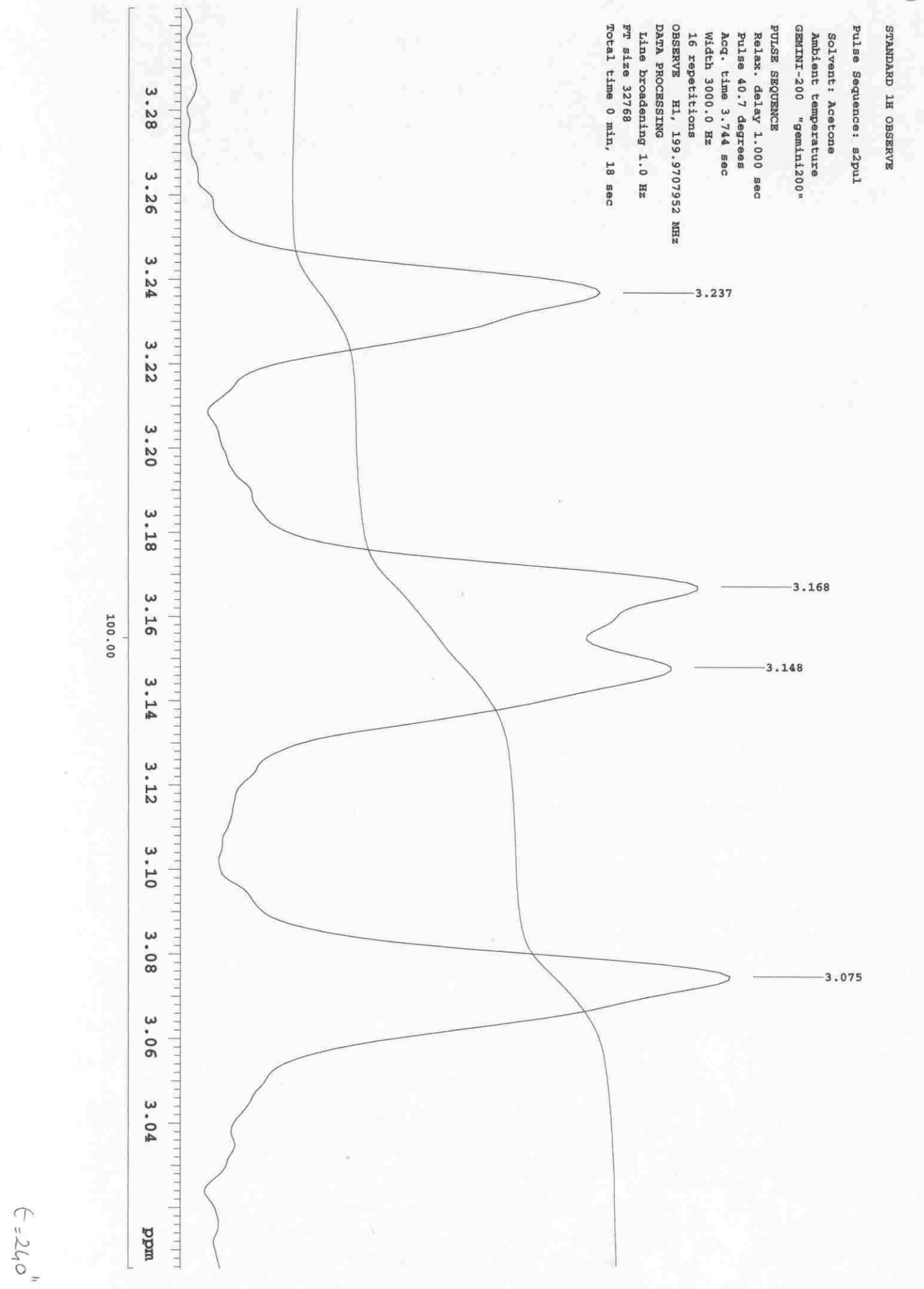


(v)

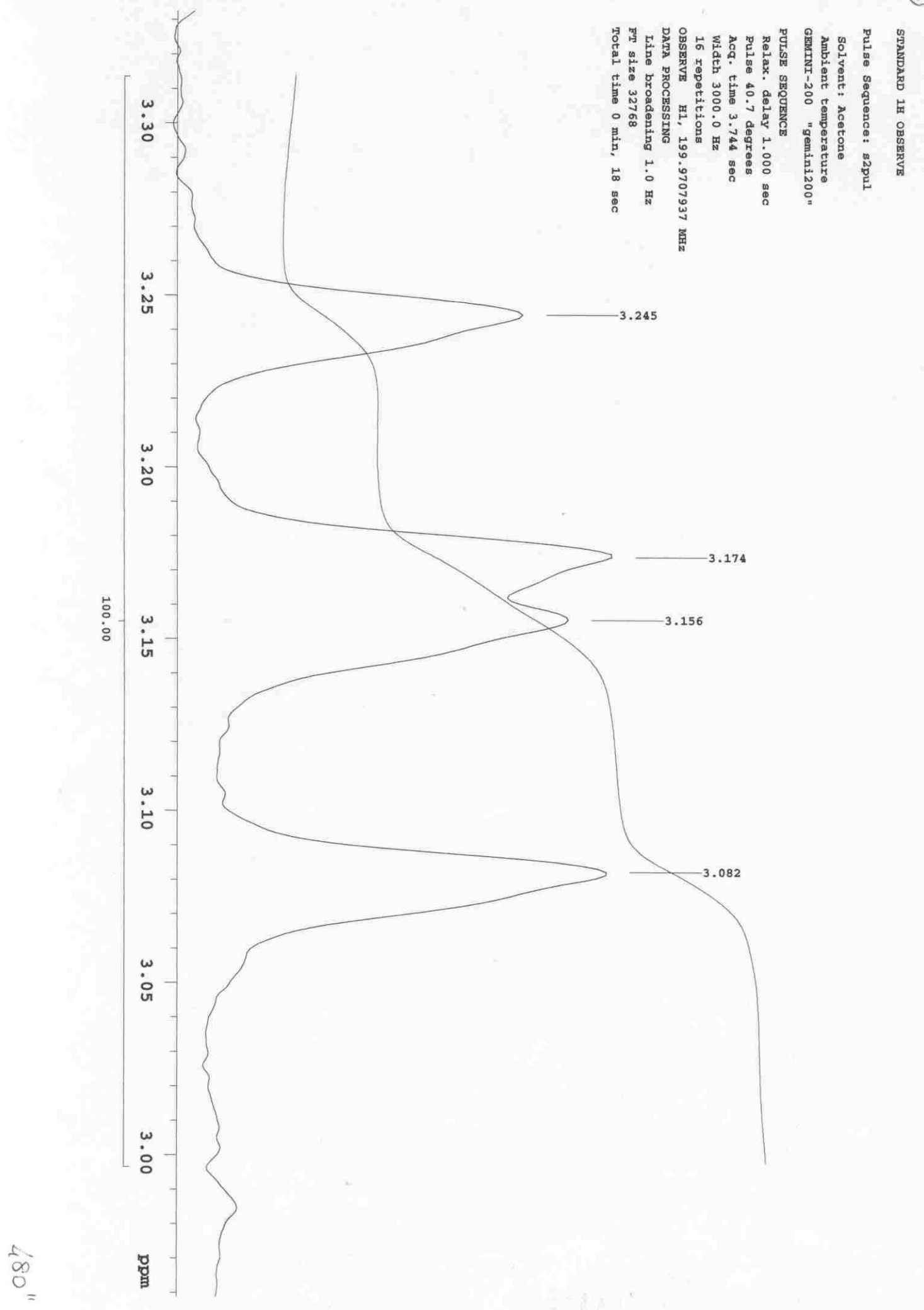




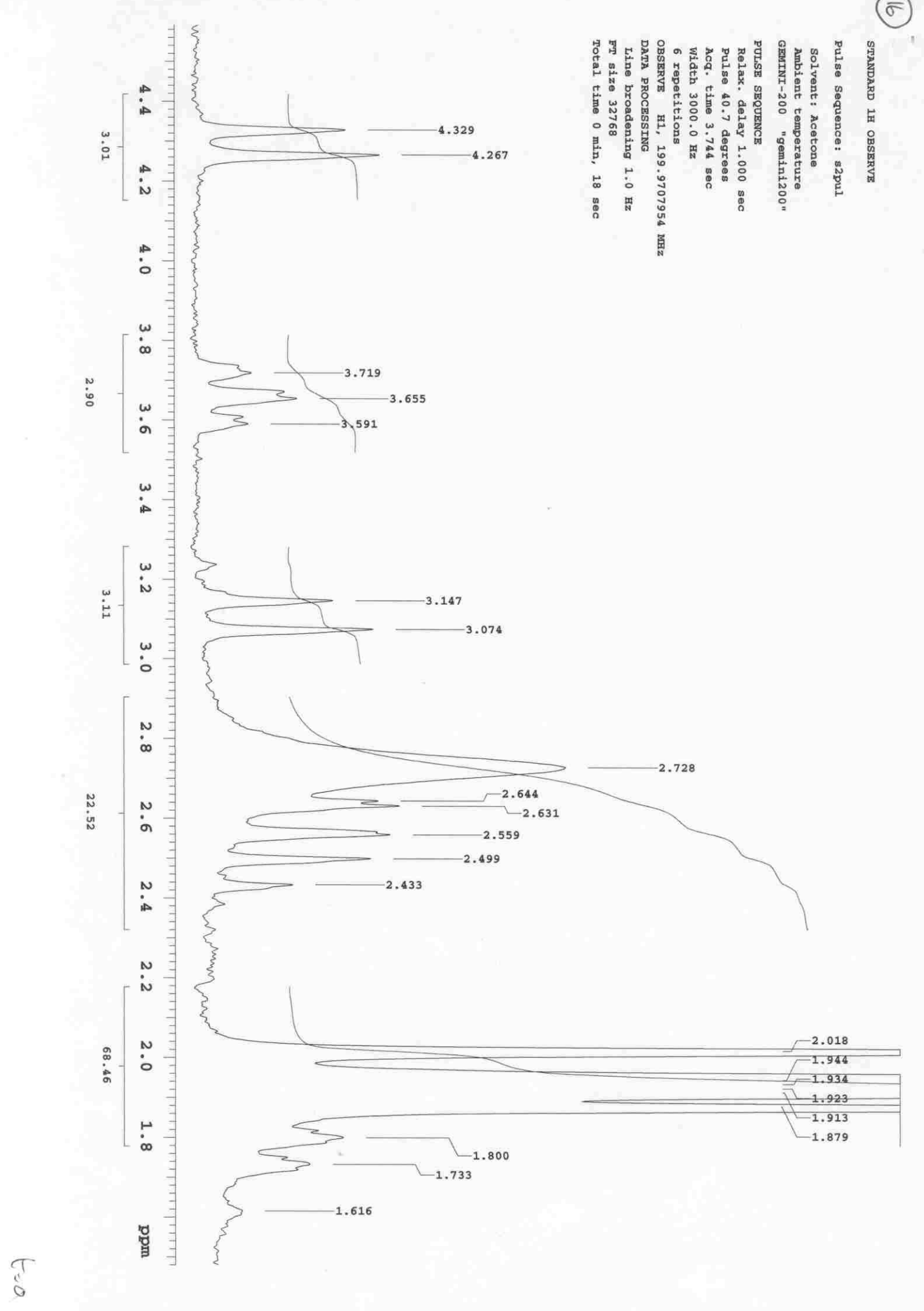




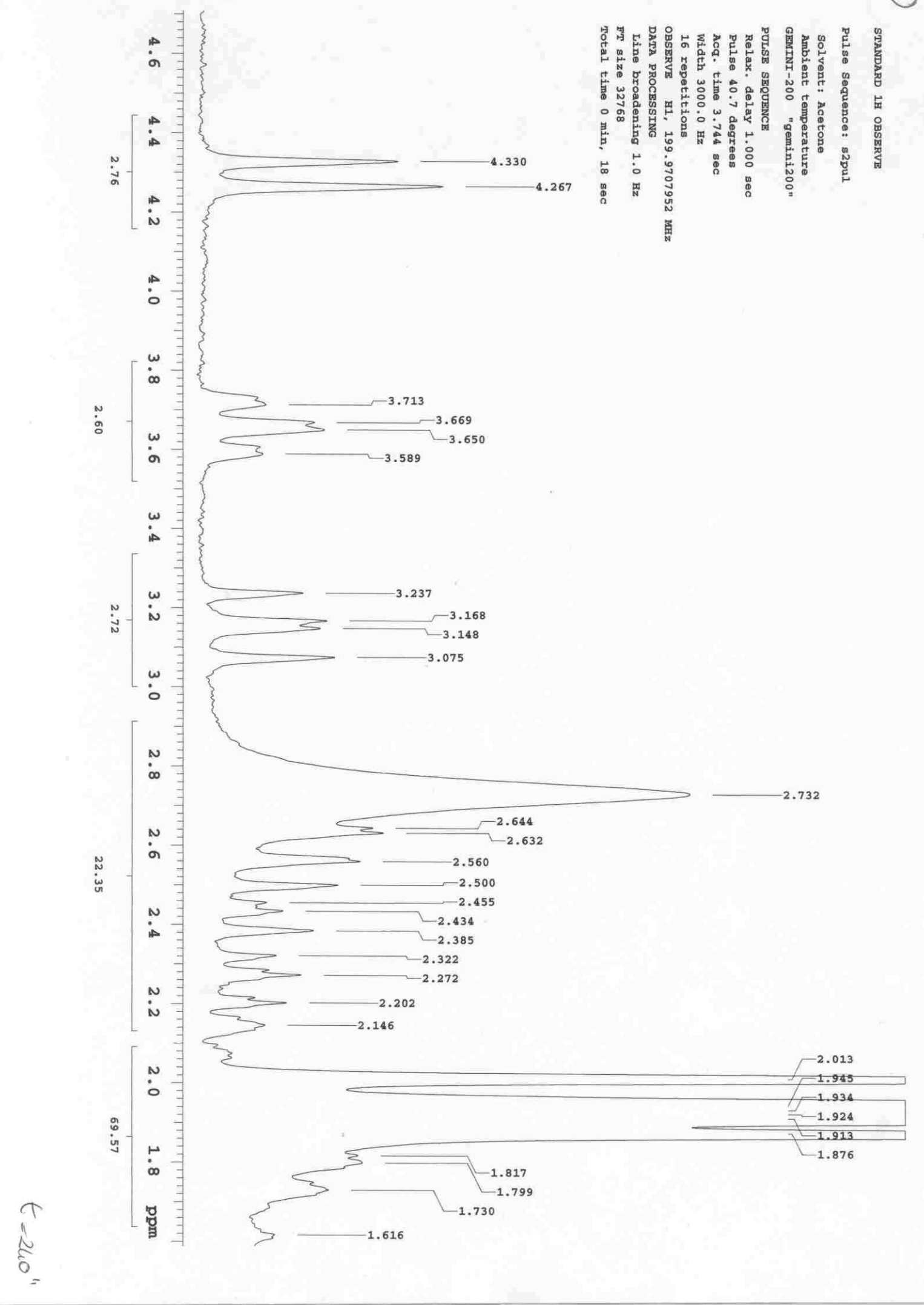


3-Methyl-4-nitro-7-oxo-9-phenyl-1-oxa-2-aza-spiro[4.5]dec-2-ene-8-carboxylic acid ethyl ester 5b

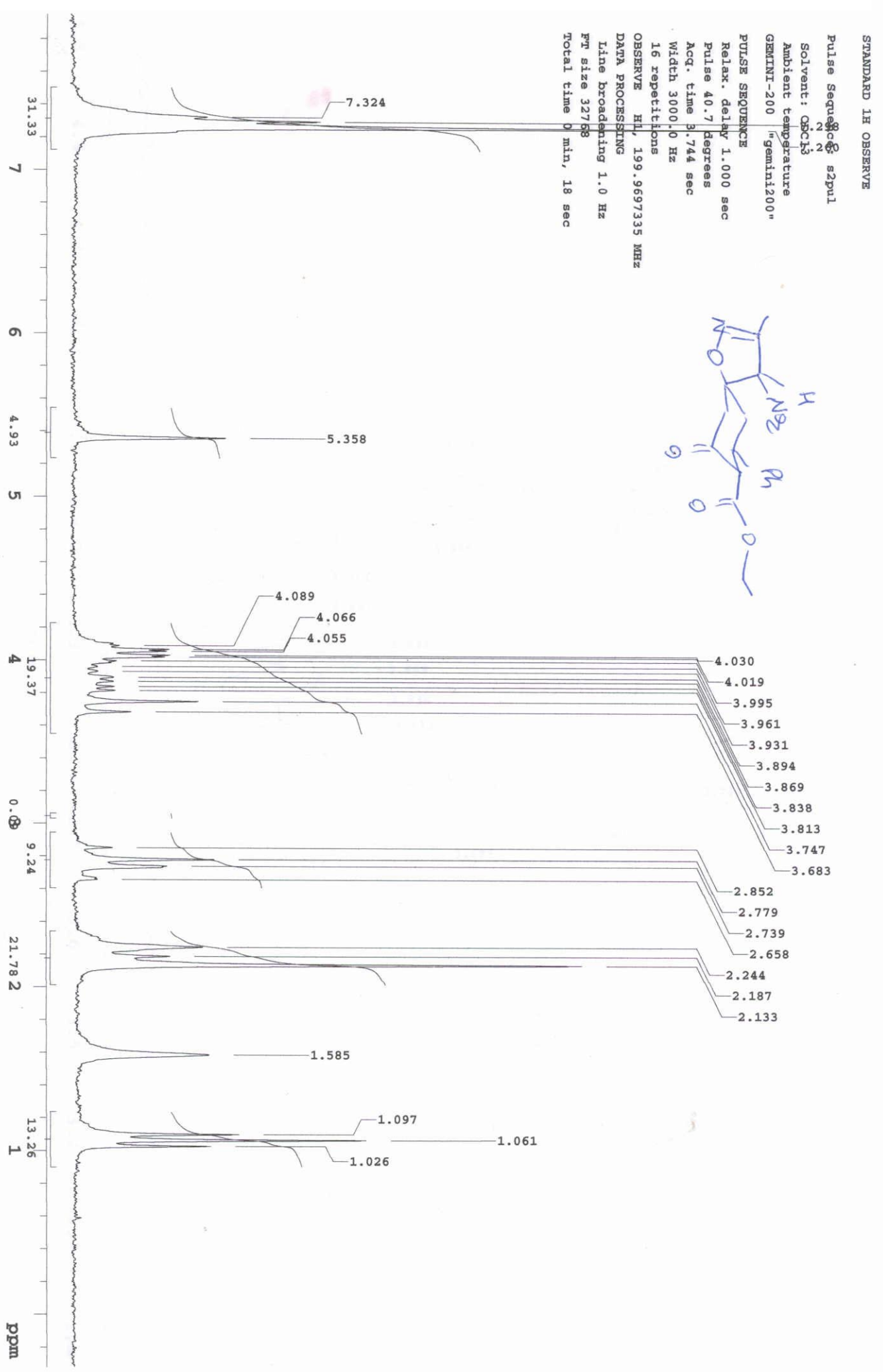


3-Methyl-4-nitro-9-phenyl-1-oxa-2-aza-spiro[4.5]dec-2-en-7-one 5c

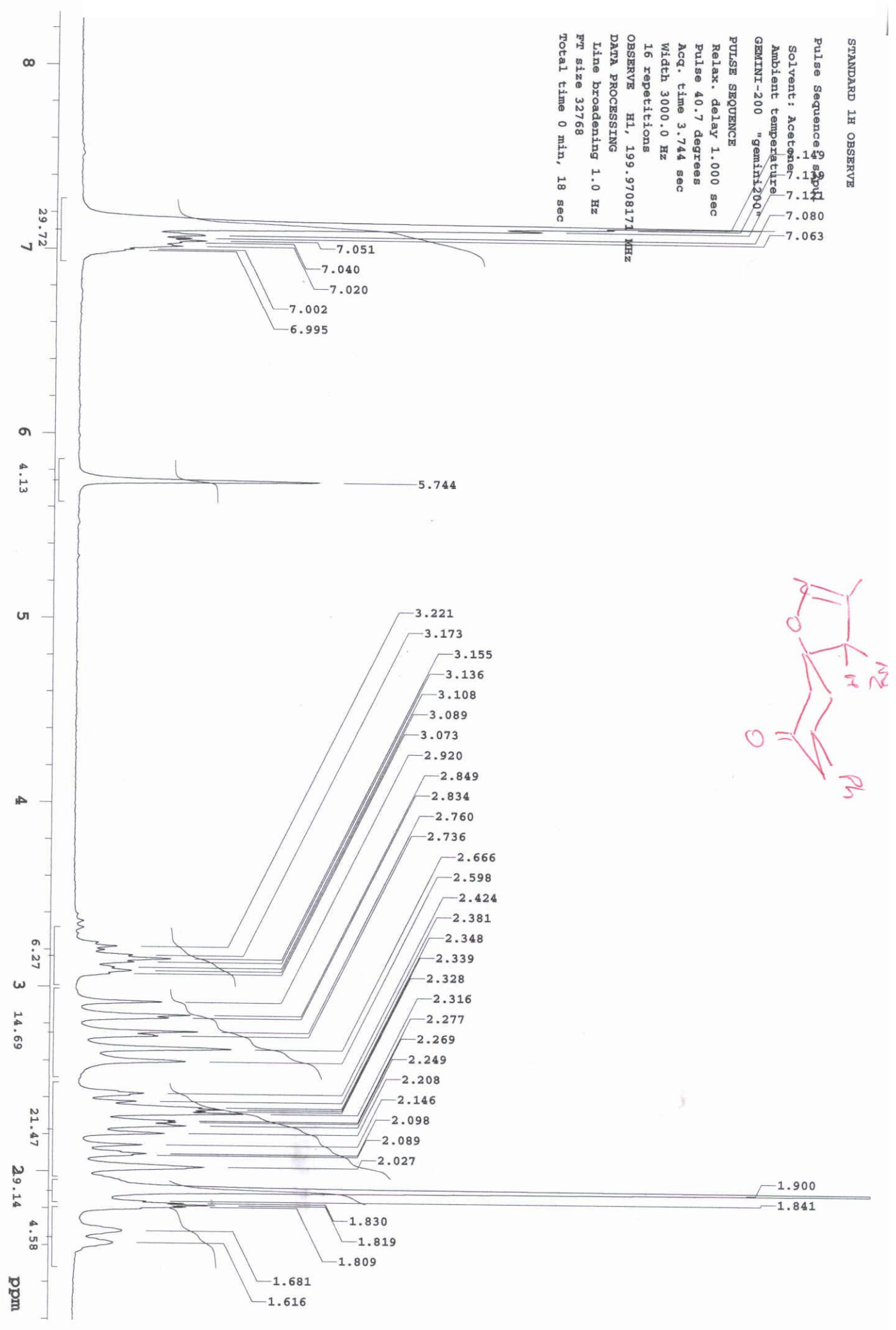


8-Acetyl-3-methyl-4-nitro-9-(4-nitro-phenyl)-1-oxa-2-aza-spiro[4.5]dec-2-en-7-one 5d

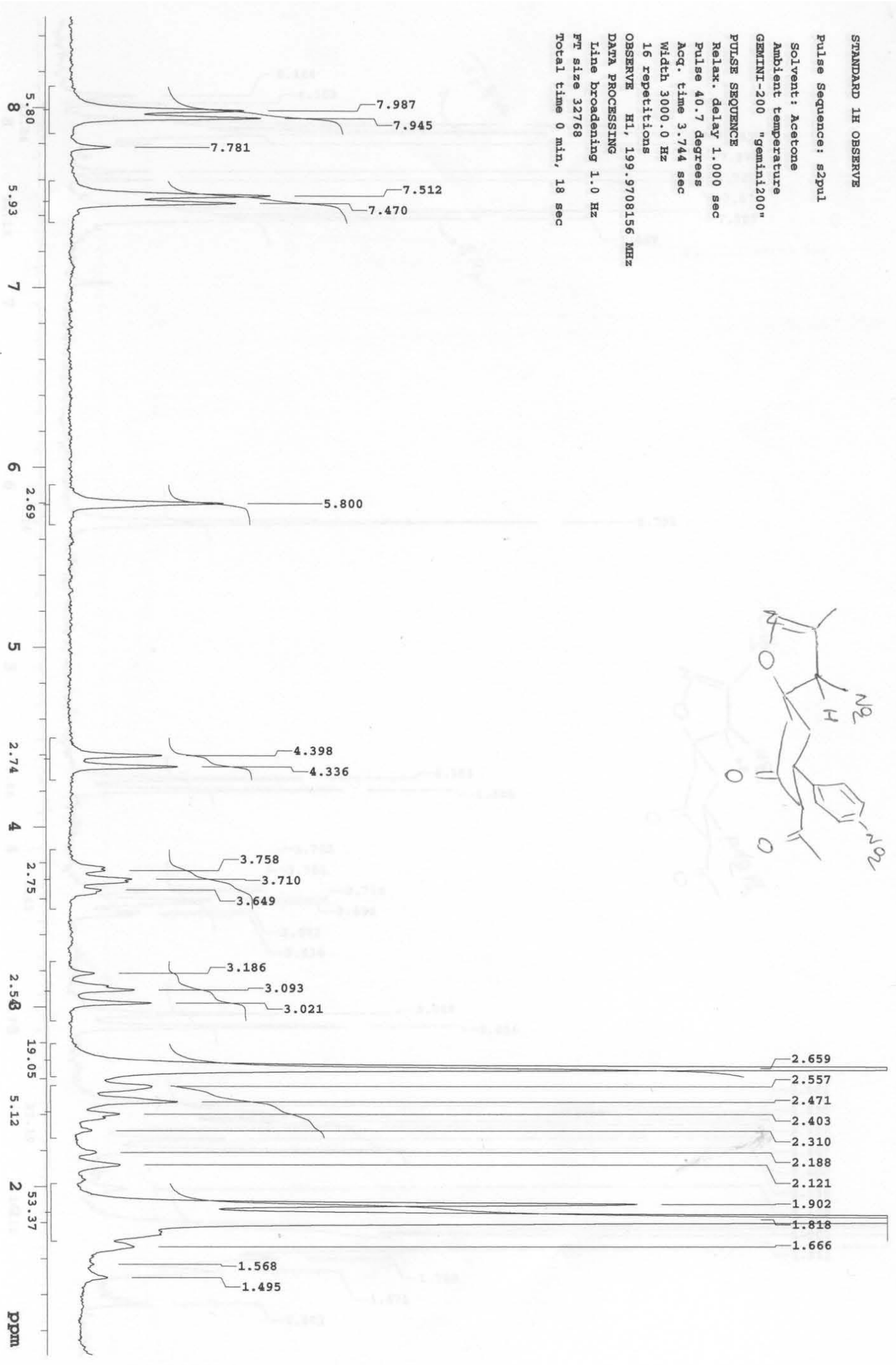


3-Methyl-4-nitro-7-(4-nitro-phenyl)-9-oxo-1-oxa-2-aza-spiro[4.5]dec-2-ene-8-carboxylic acid ethyl ester 5e
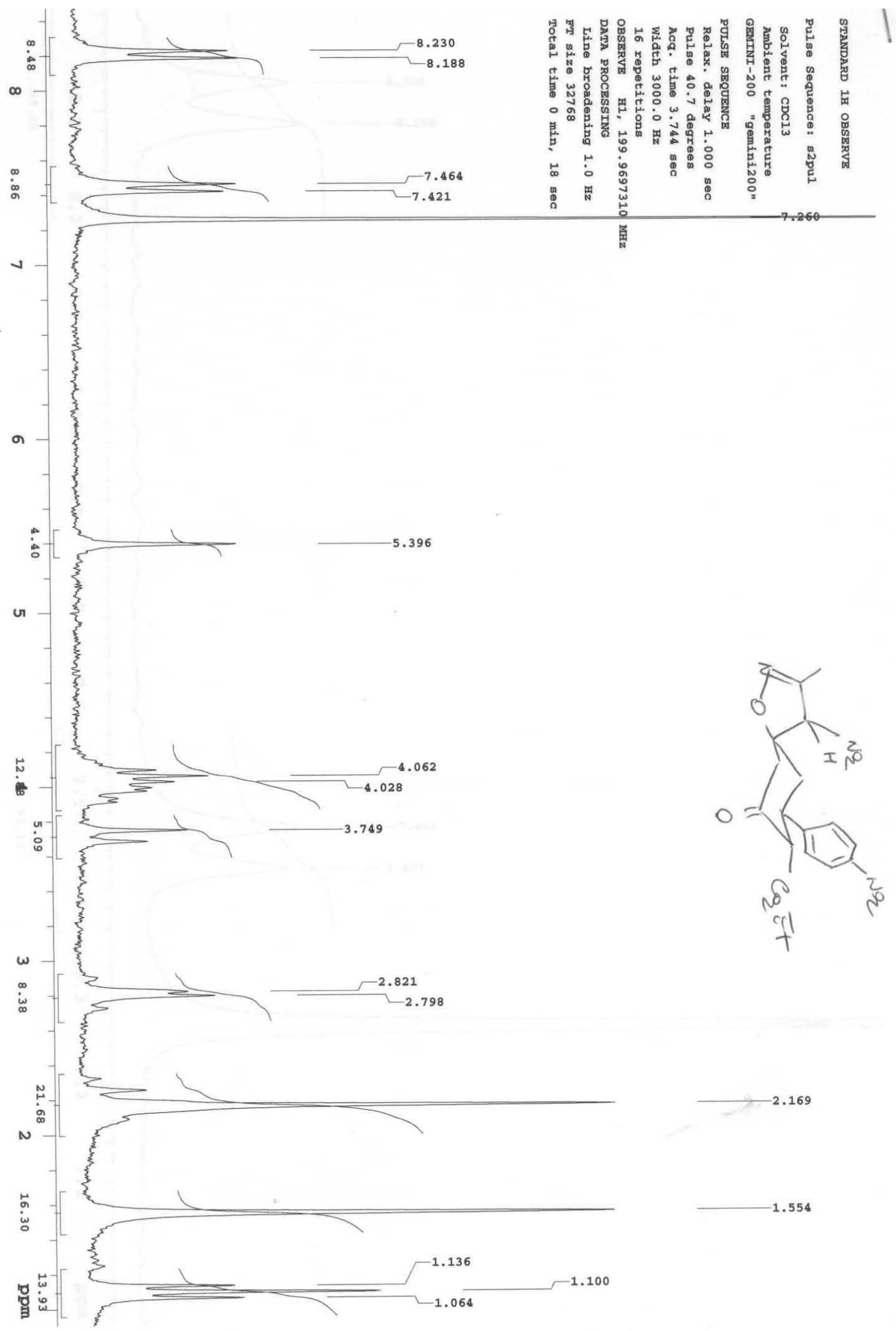
3-Methyl-4-nitro-9-(4-nitro-phenyl)-1-oxa-2-aza-spiro[4.5]dec-2-en-7-one 5f
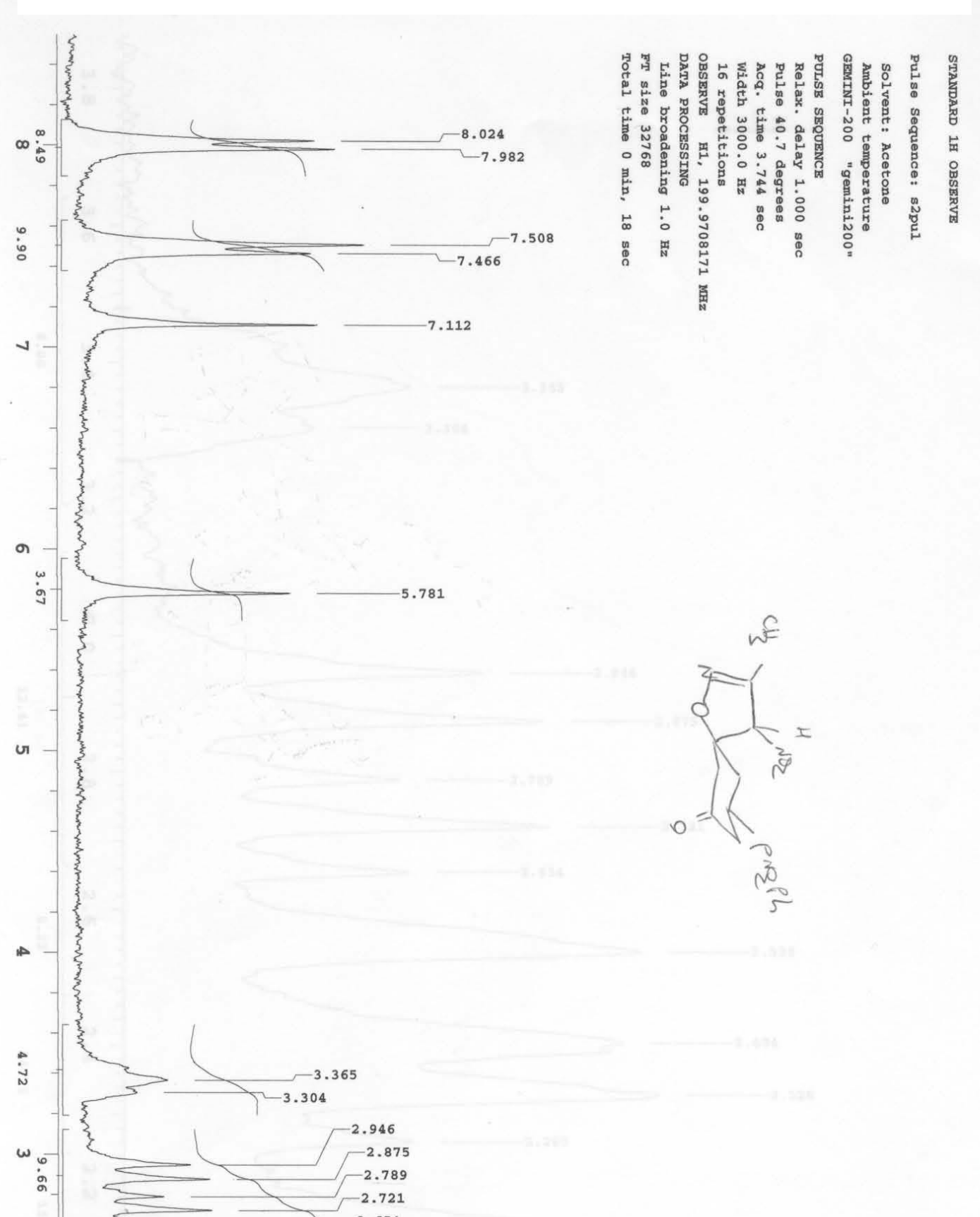

is

is

$+$

Ni⿱n.

ป

-
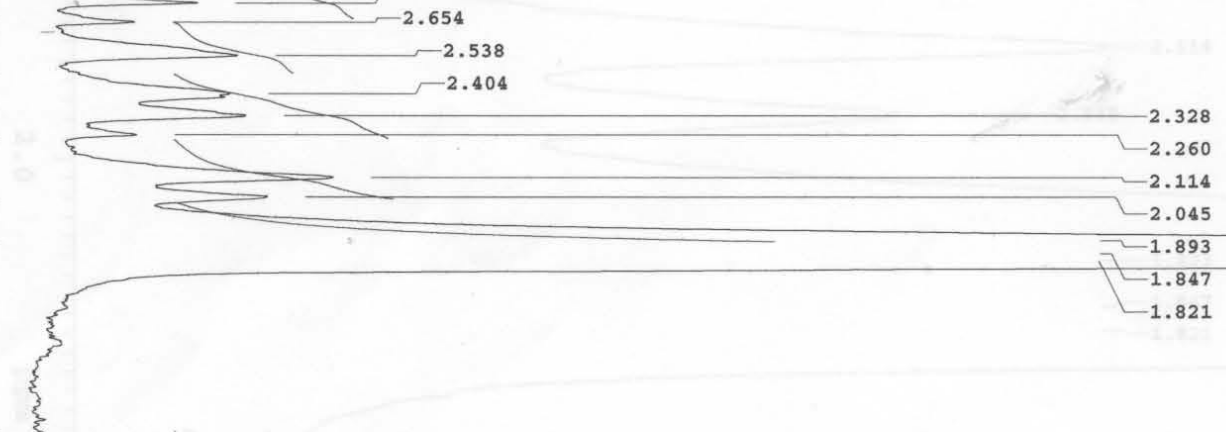

893 
8-Acetyl-9-(4-chloro-phenyl)-3-methyl-4-nitro-1-oxa-2-aza-spiro[4.5]dec-2-en-7-one 5g

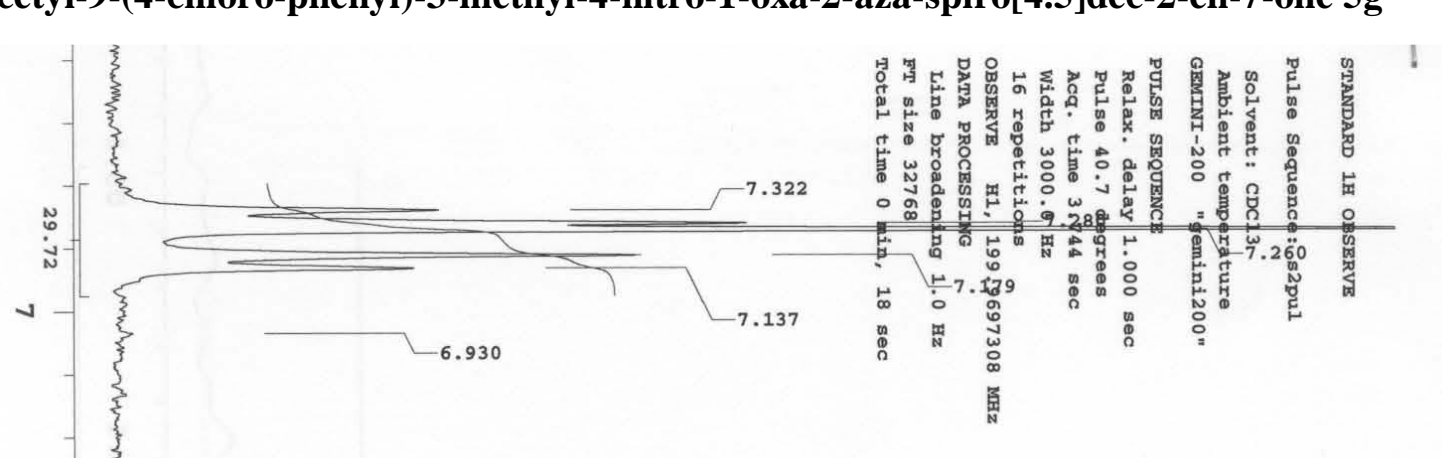

a

$$
\text { ì }
$$

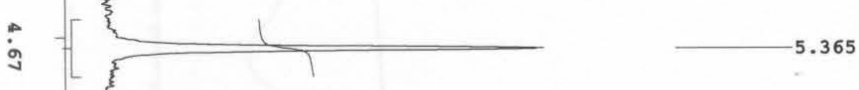

v
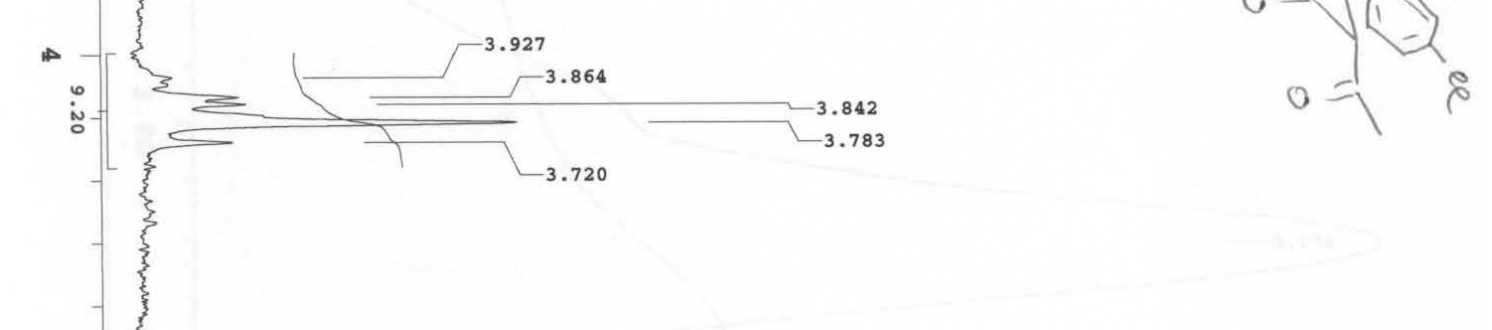

$\omega$

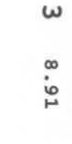

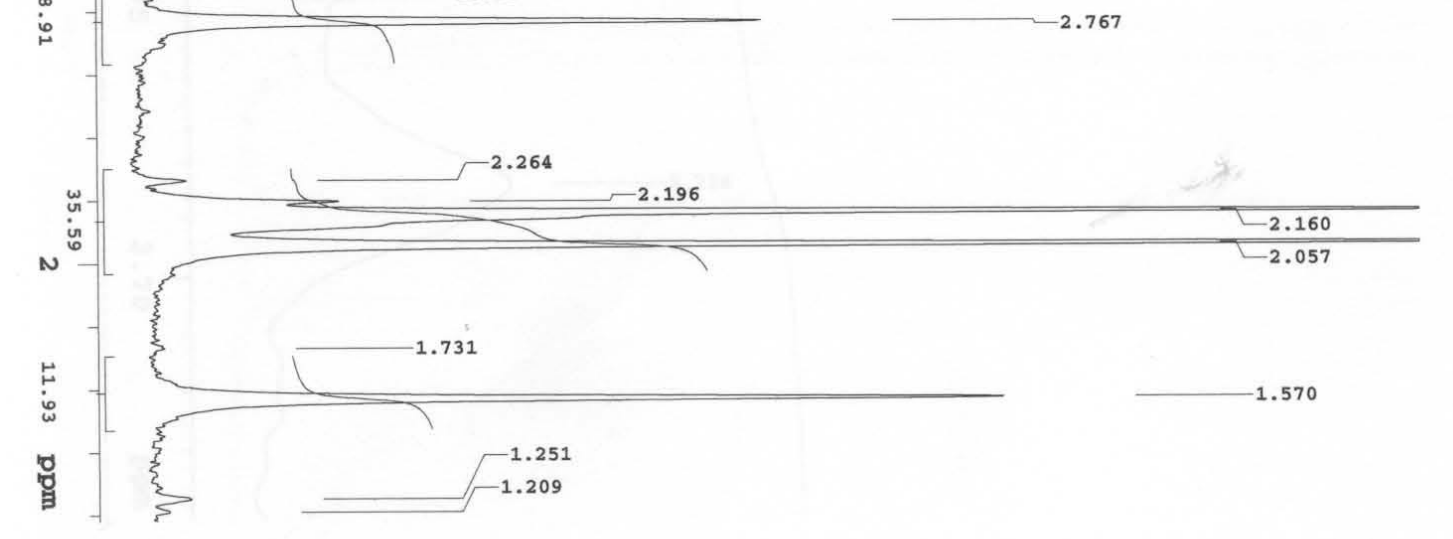

( 
7-(4-Chloro-phenyl)-3-methyl-4-nitro-9-oxo-1-oxa-2-aza-spiro[4.5]dec-2-ene-8-carboxylic acid ethyl ester $5 \mathrm{~h}$

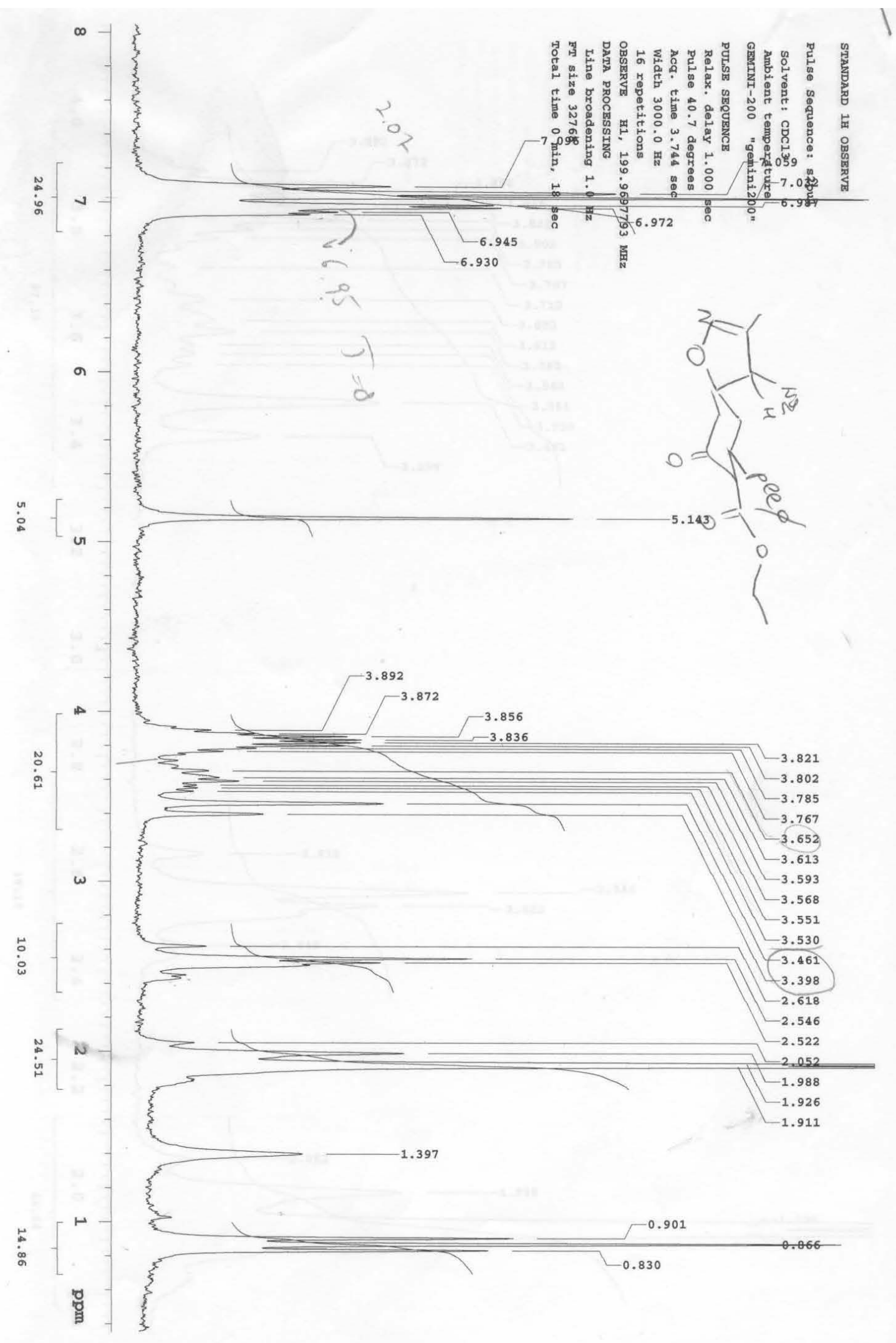


9-(4-Chloro-phenyl)-3-methyl-4-nitro-1-oxa-2-aza-spiro[4.5]dec-2-en-7-one 5i

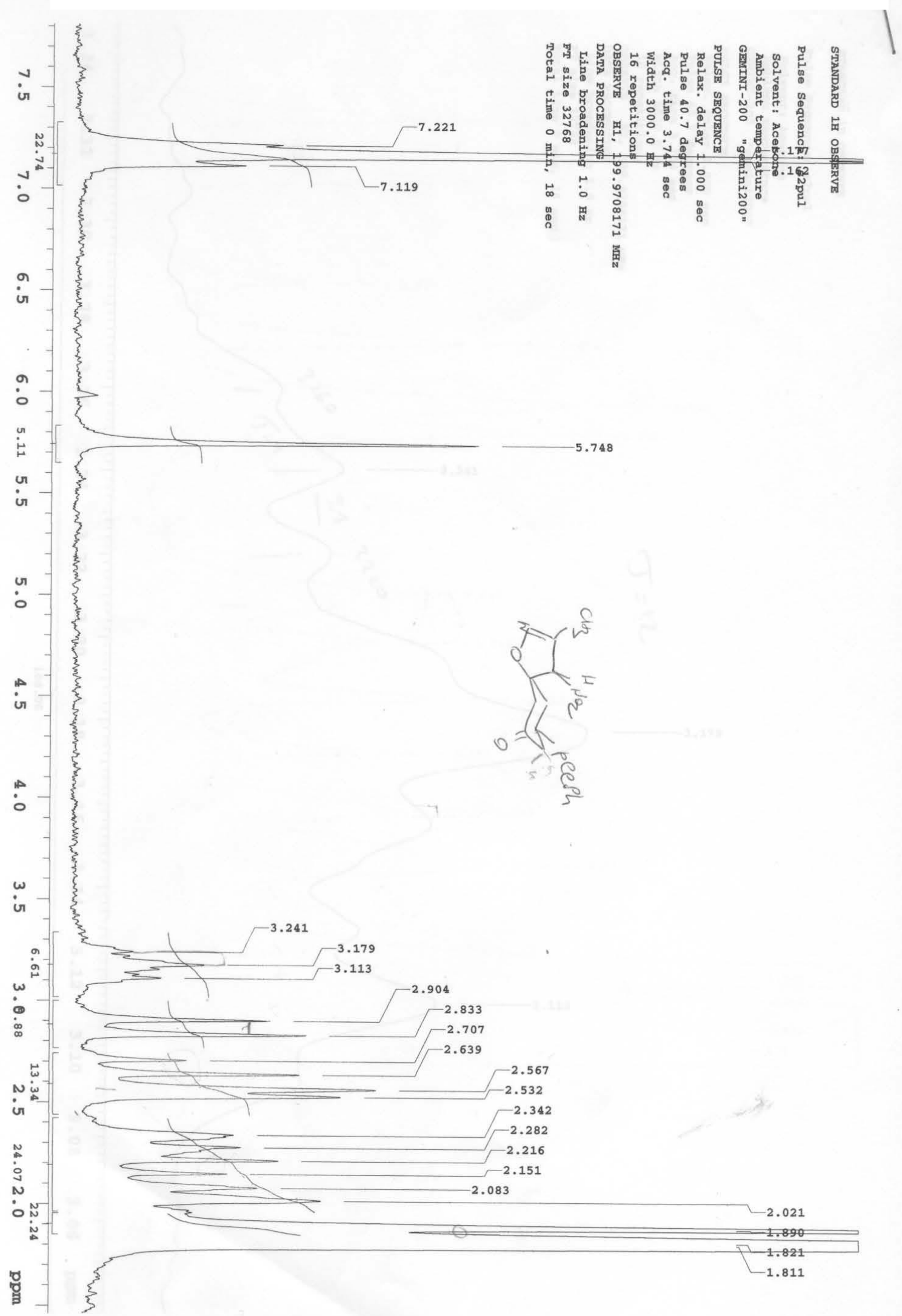


8-Acetyl-9-(2-chloro-phenyl)-3-methyl-4-nitro-1-oxa-2-aza-spiro[4.5]dec-2-en-7-one 5k

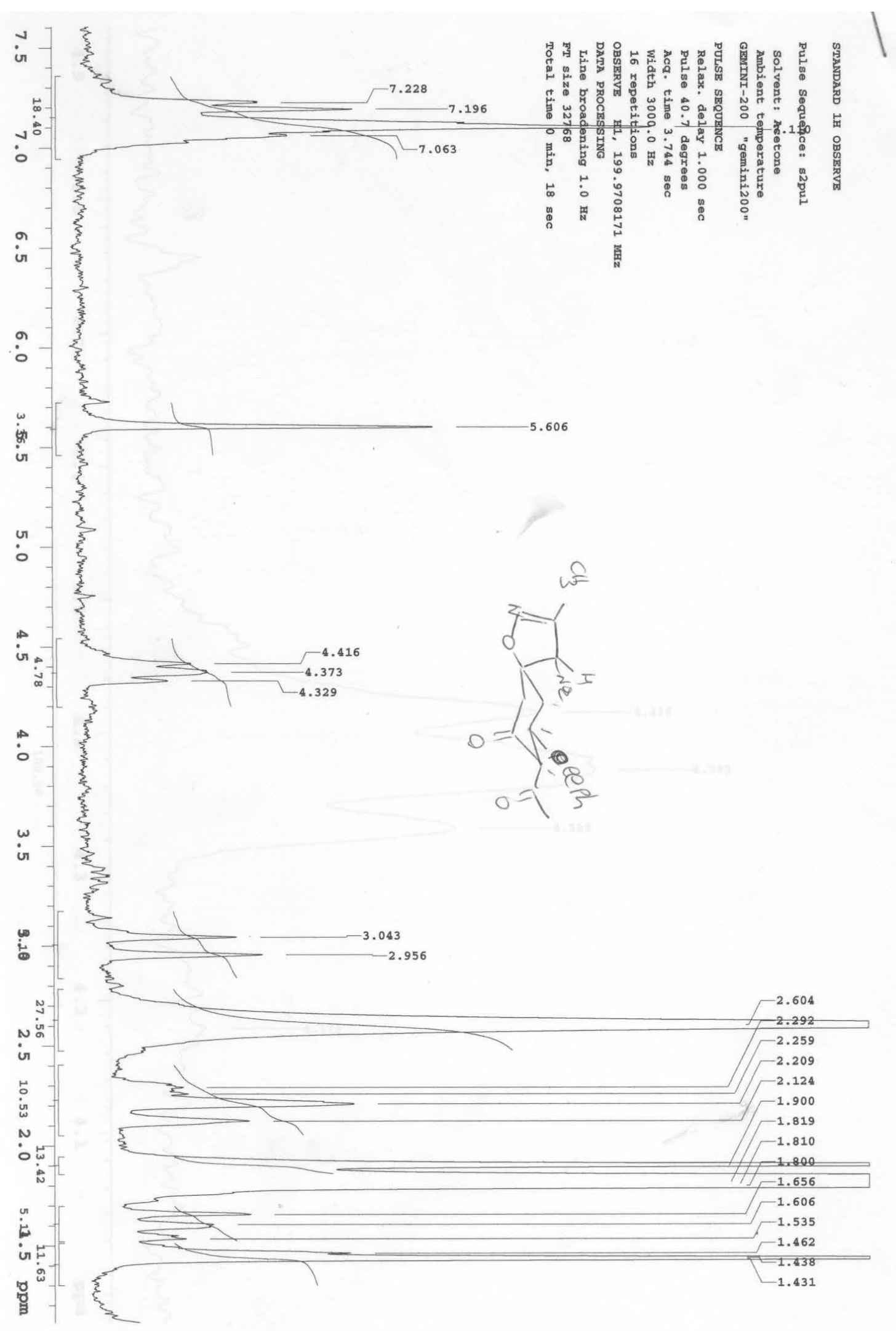


7-(2-Chloro-phenyl)-3-methyl-4-nitro-9-oxo-1-oxa-2-aza-spiro[4.5]dec-2-ene-8-carboxylic acid ethyl ester 51

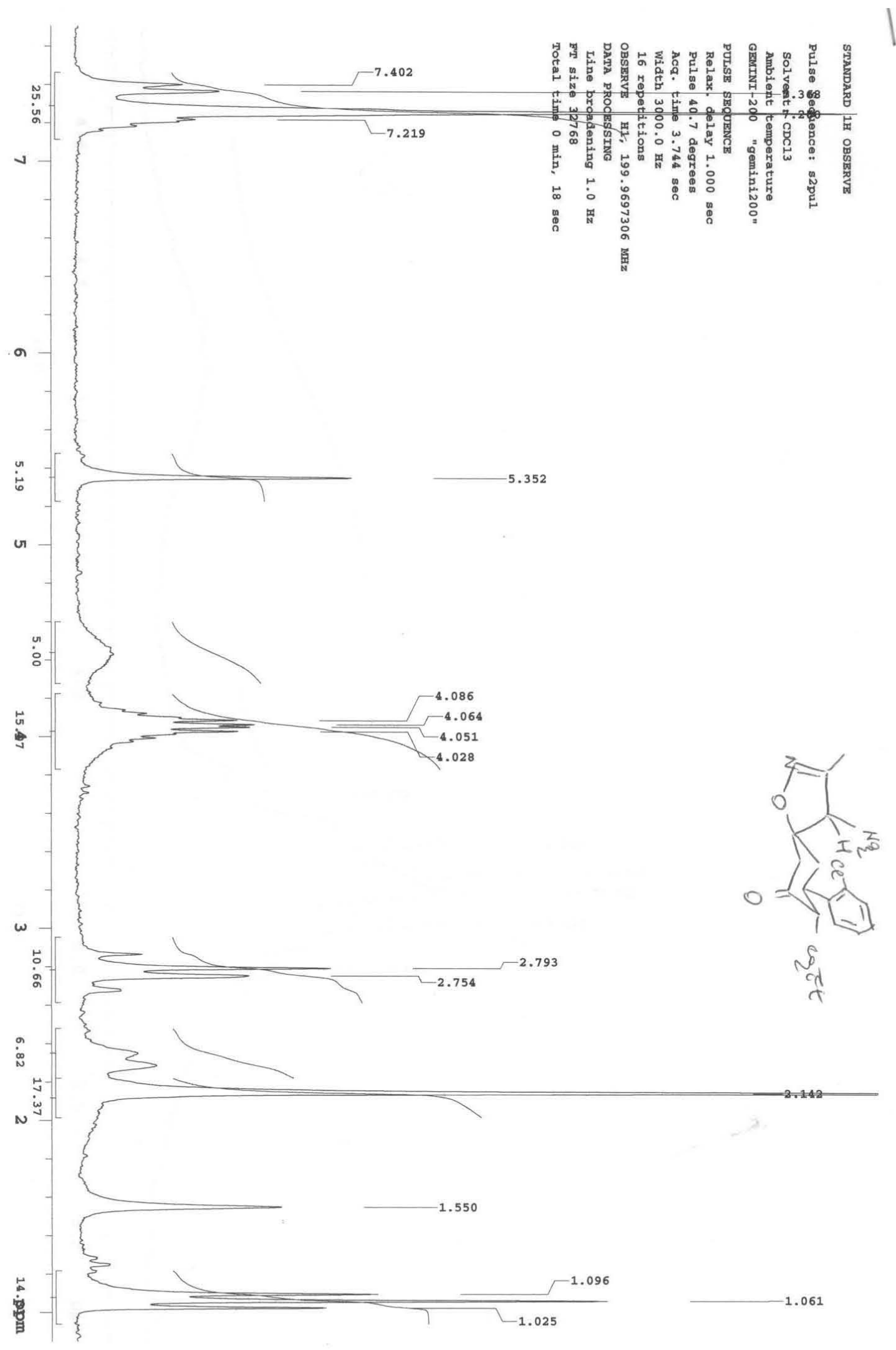


9-(2-Chloro-phenyl)-3-methyl-4-nitro-1-oxa-2-aza-spiro[4.5]dec-2-en-7-one $5 \mathrm{~m}$

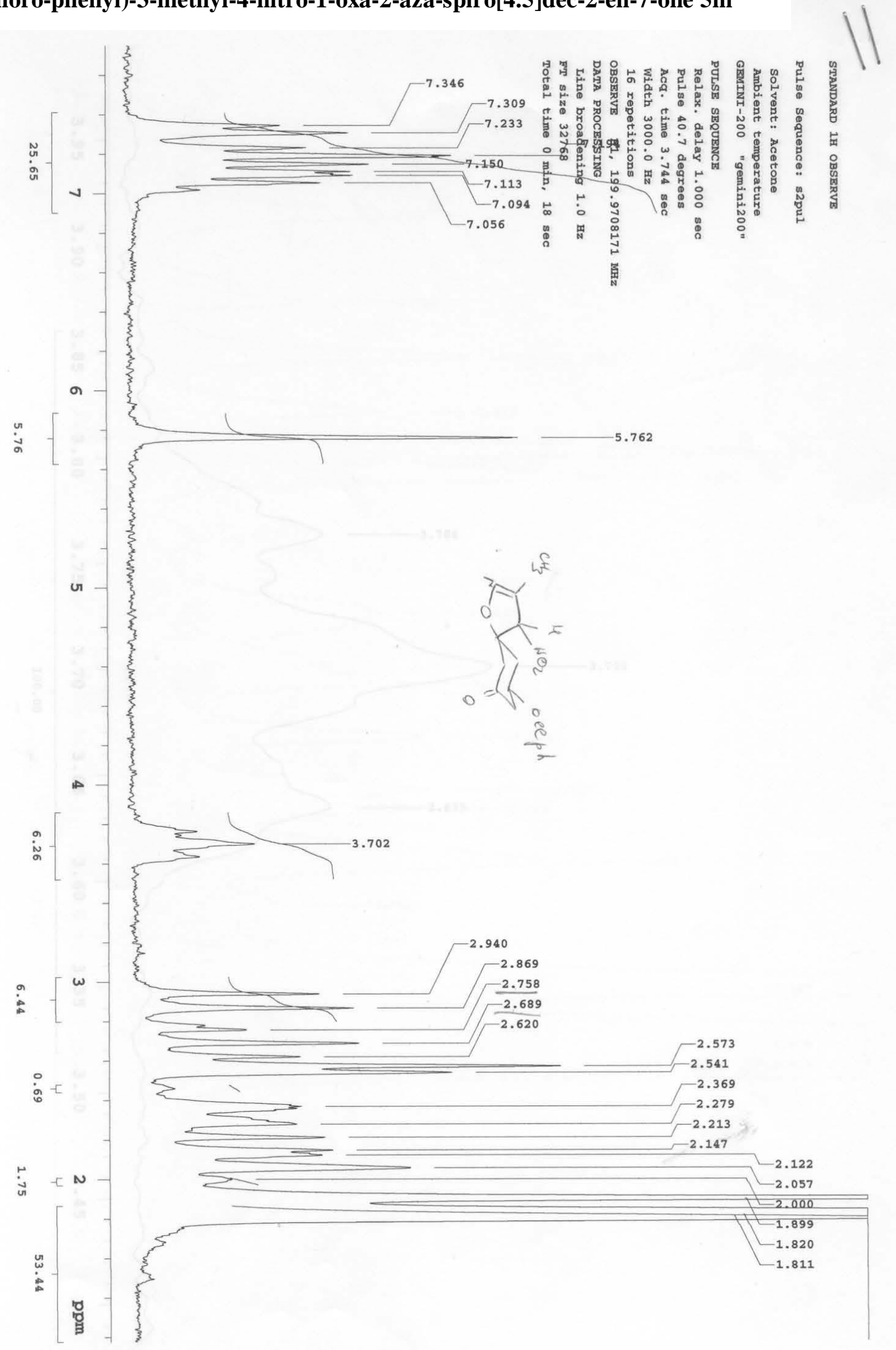


8-Acetyl-9-(4-methoxy-phenyl)-3-methyl-4-nitro-1-oxa-2-aza-spiro[4.5]dec-2-en-7-one 5n

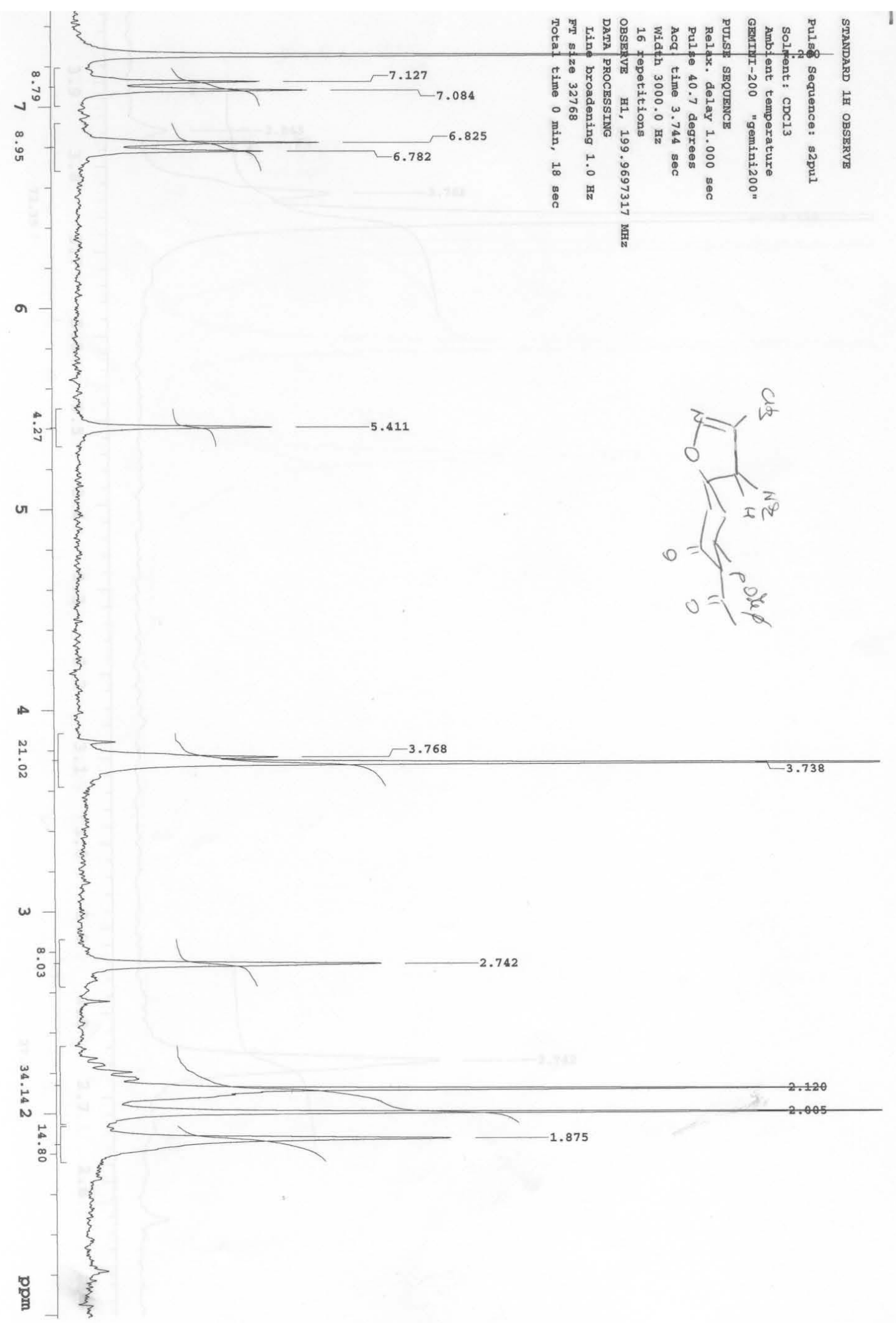


7-(4-Methoxy-phenyl)-3-methyl-4-nitro-9-oxo-1-oxa-2-aza-spiro[4.5]dec-2-ene-8-carboxylic acid ethyl ester 5o

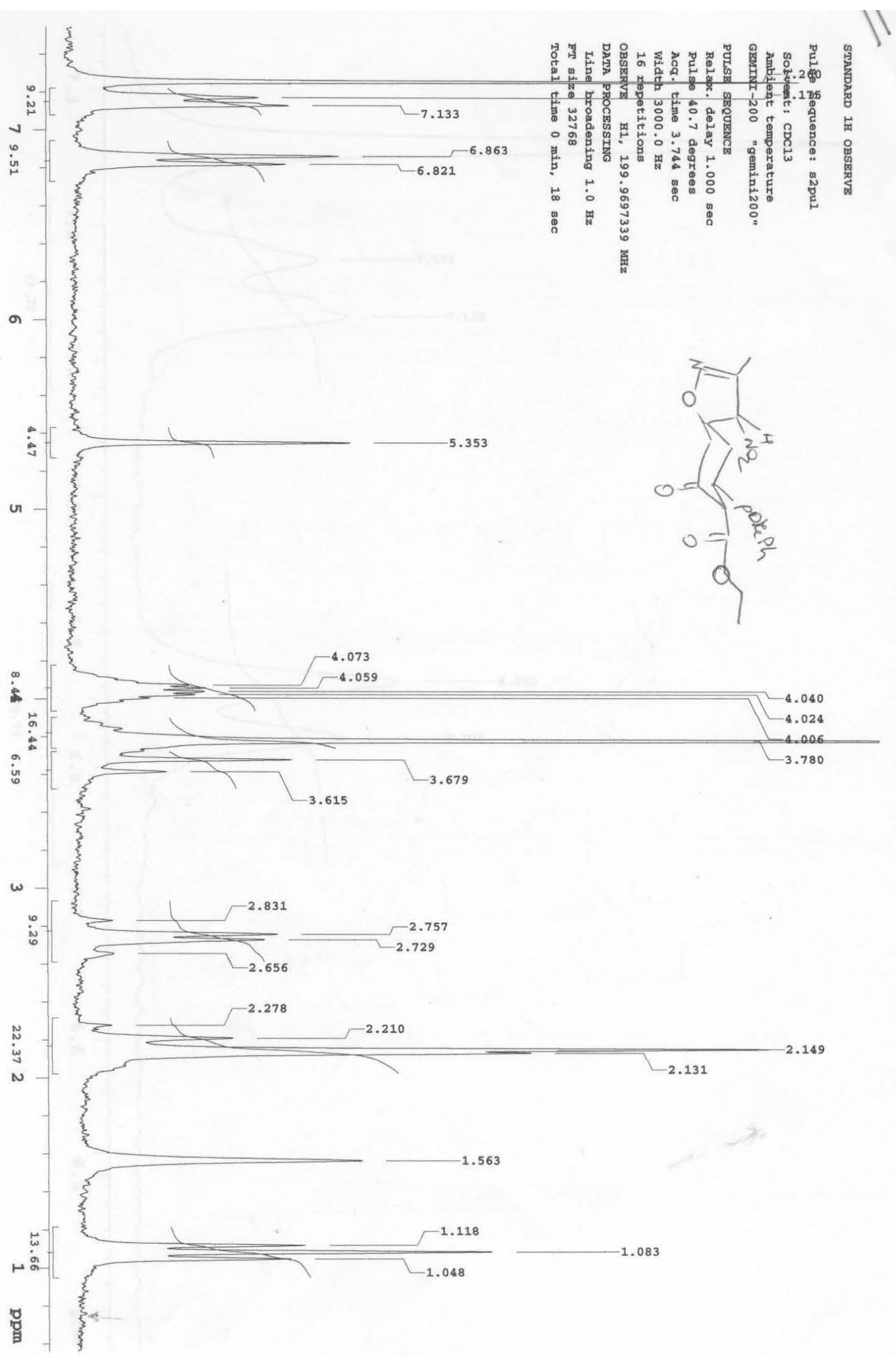


9-(4-Methoxy-phenyl)-3-methyl-4-nitro-1-oxa-2-aza-spiro[4.5]dec-2-en-7-one 5p

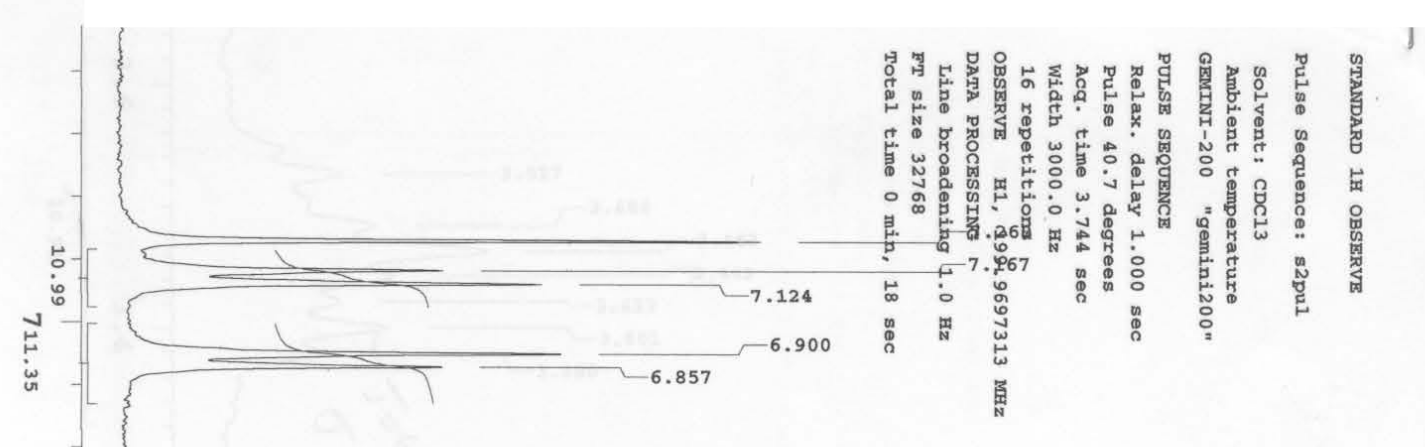

a

v

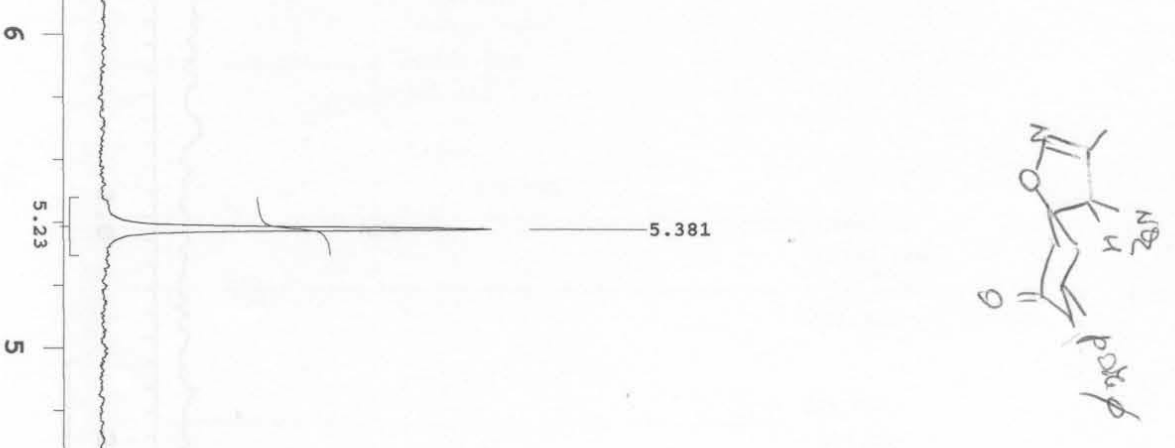

$$
\text { : }
$$

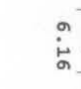




\section{3-[2-(3-Methyl-4-nitro-isoxazol-5-yl)-1-phenyl-ethyl]-pentane-2,4-dione 3a}

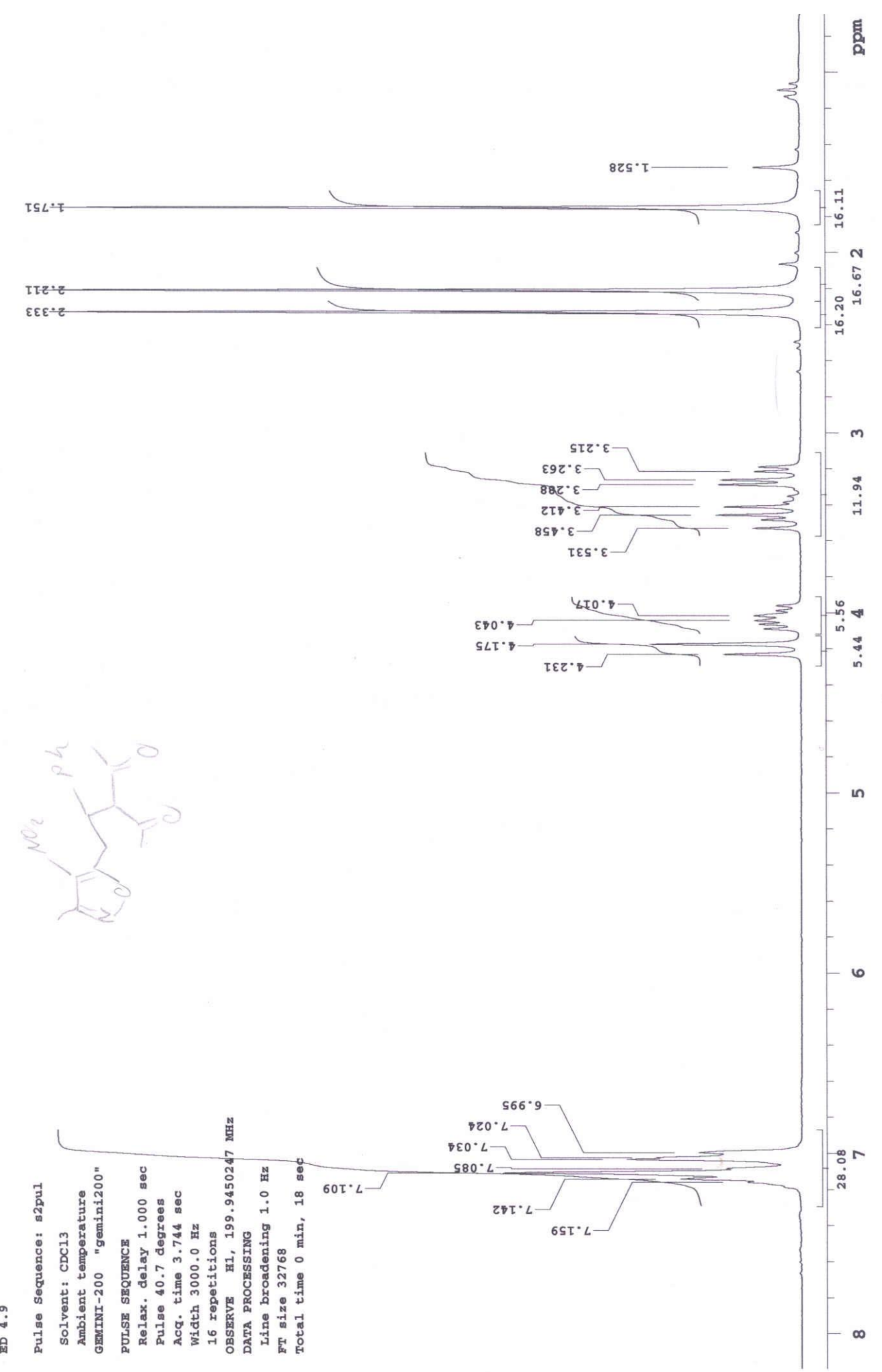




\section{2-Acetyl-4-(3-methyl-4-nitro-isoxazol-5-yl)-3-phenyl-butyric acid ethyl ester 3b}

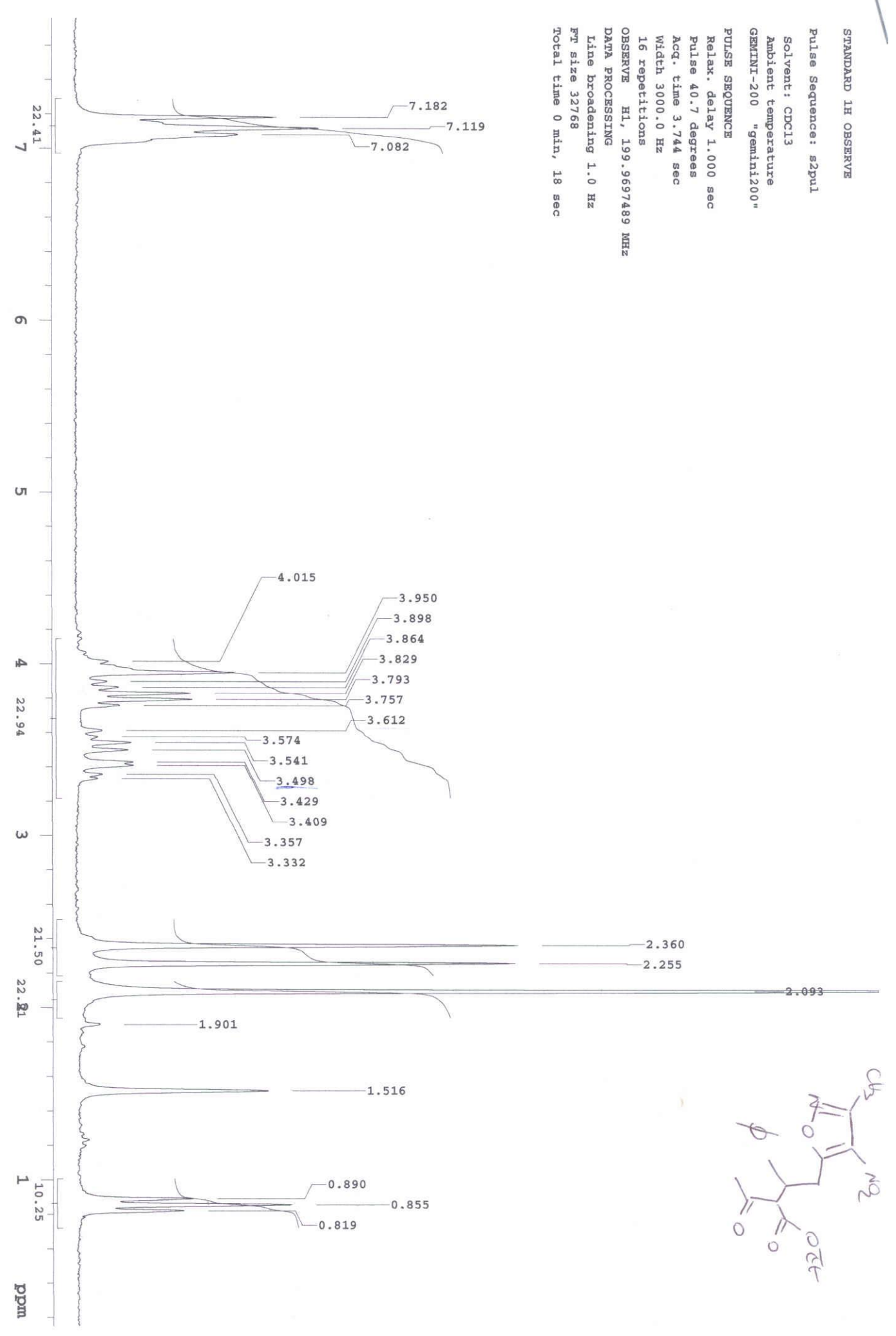


5-(3-Methyl-4-nitro-isoxazol-5-yl)-4-phenyl-pentan-2-one 3c

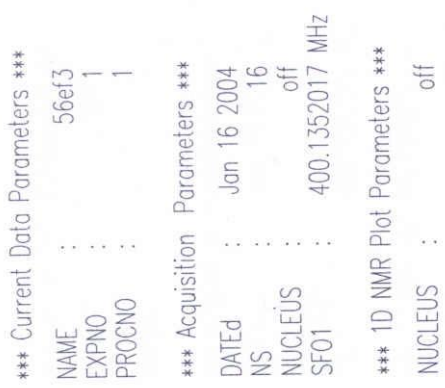

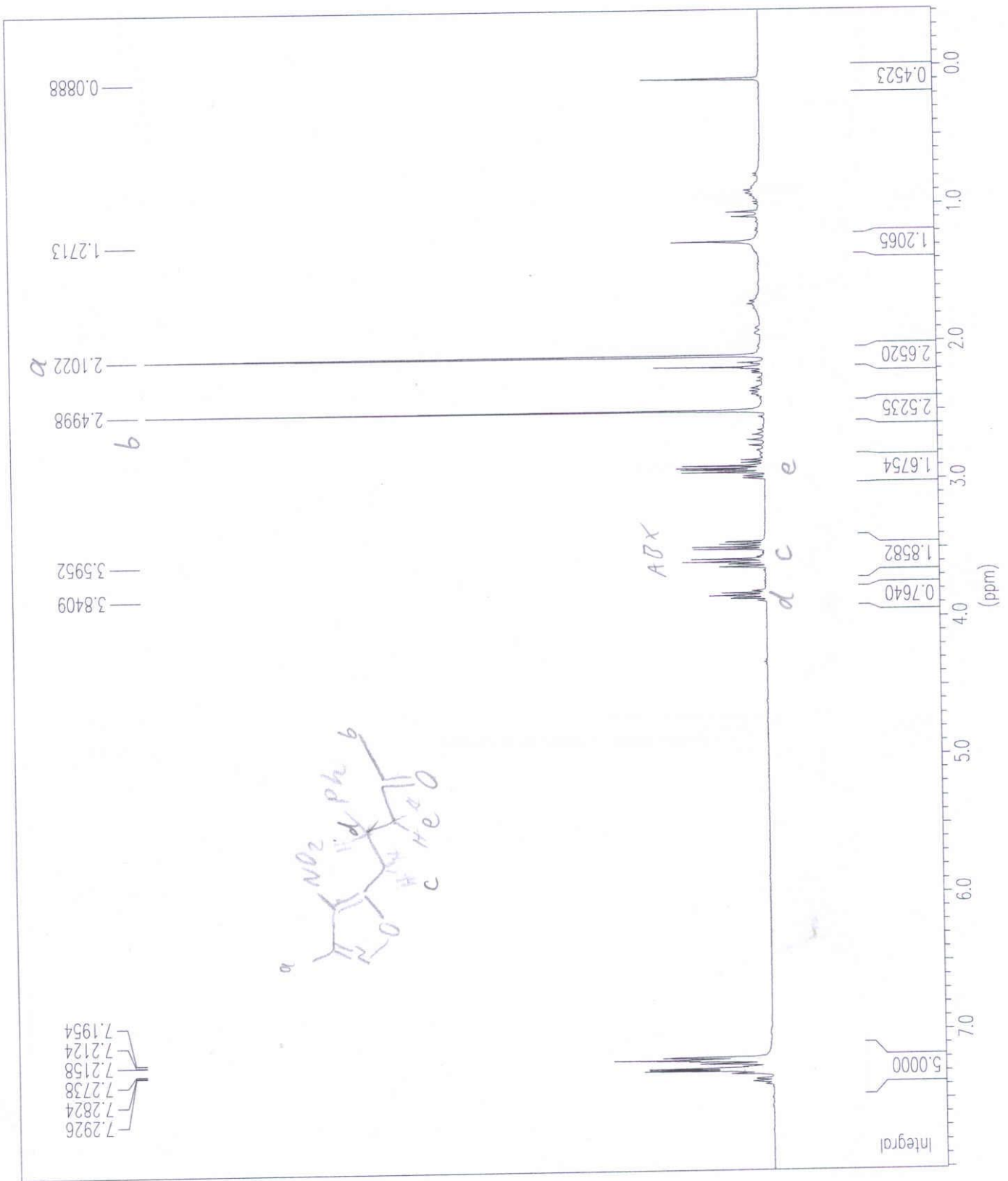


3-[2-(3-Methyl-4-nitro-isoxazol-5-yl)-1-(4-nitro-phenyl)-ethyl]-pentane-2,4-dione 3d

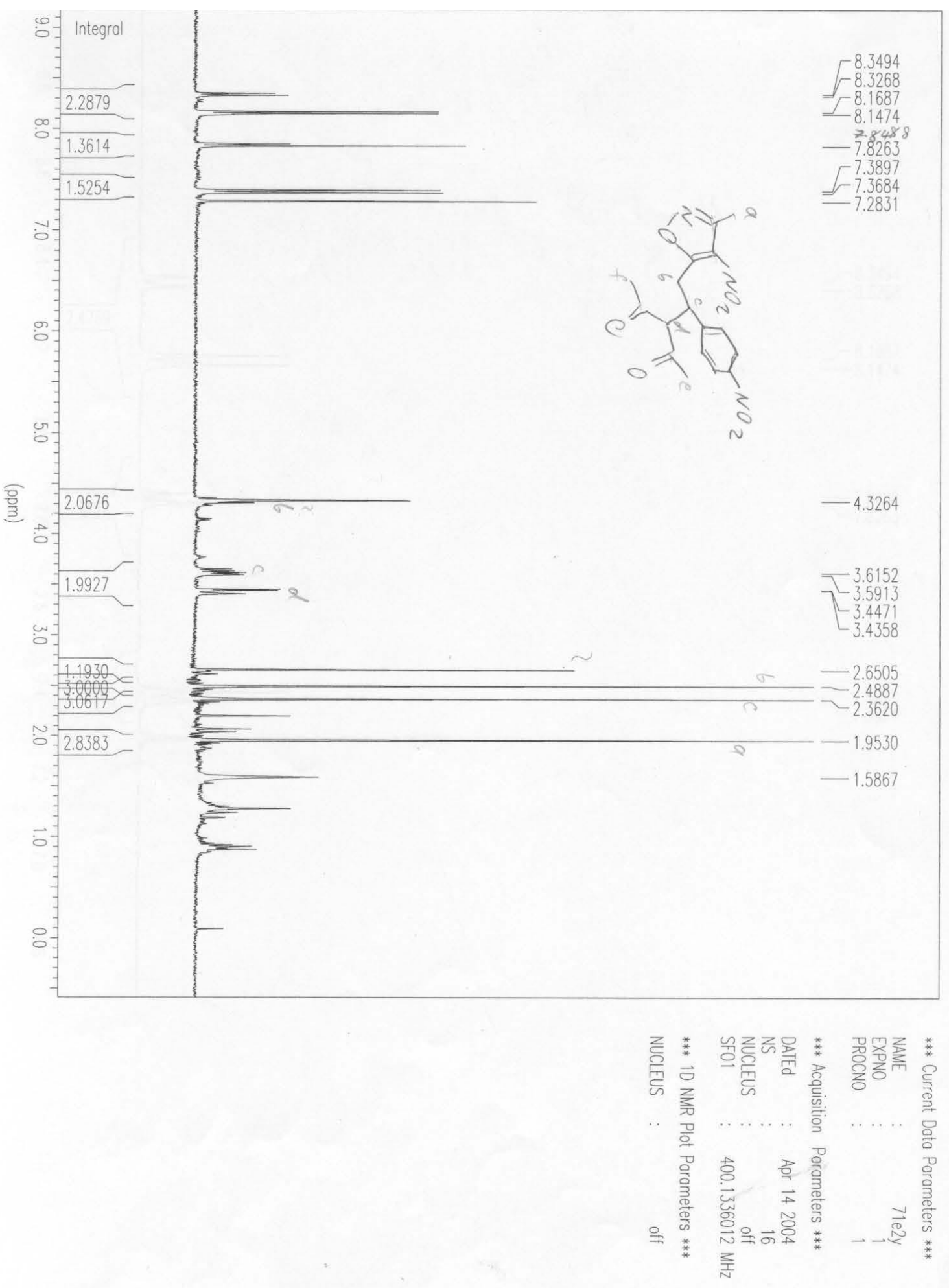


2-Acetyl-4-(3-methyl-4-nitro-isoxazol-5-yl)-3-(4-nitro-phenyl)-butyric acid ethyl ester 3e

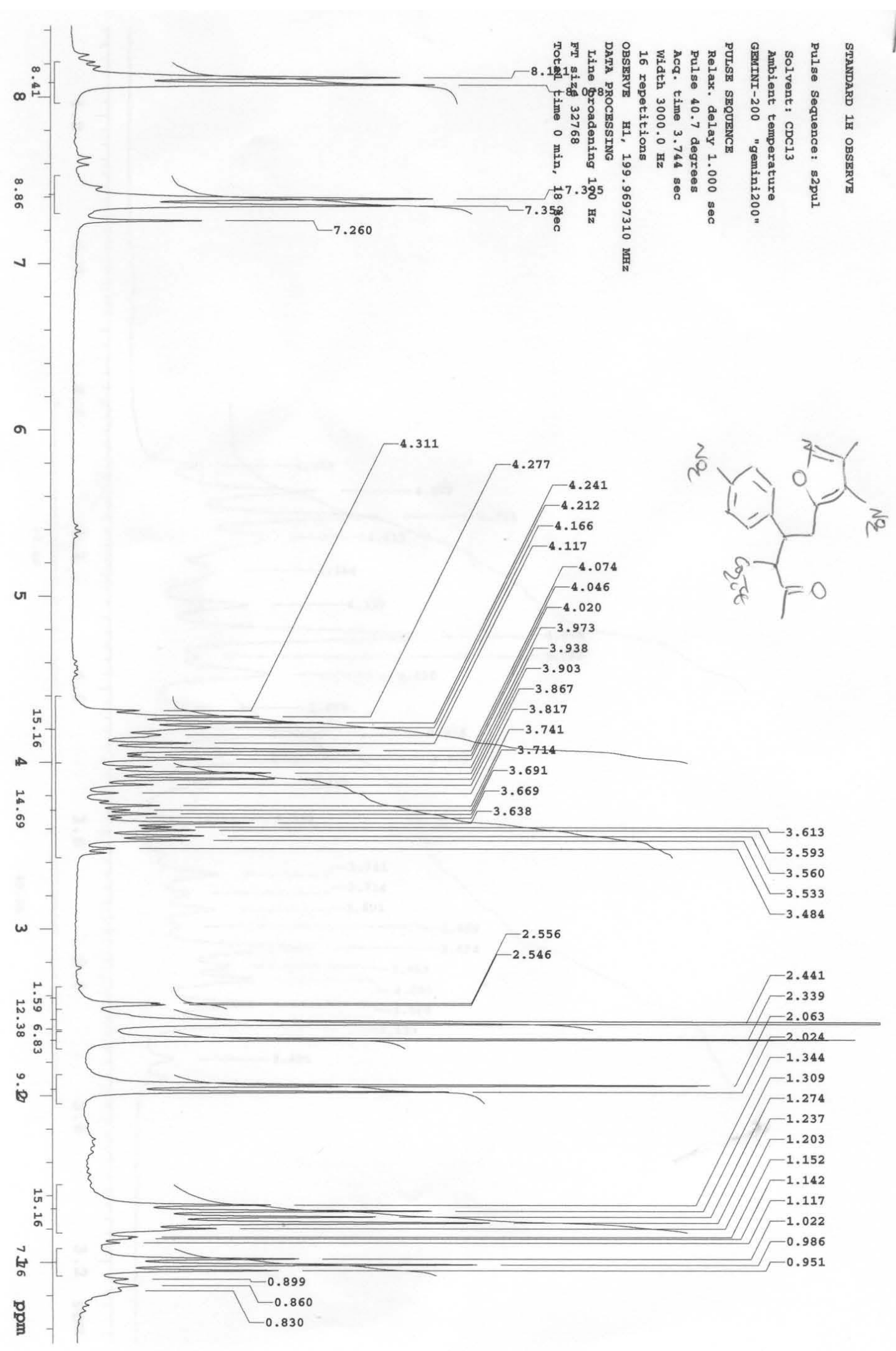


5-(3-Methyl-4-nitro-isoxazol-5-yl)-4-(4-nitro-phenyl)-pentan-2-one 3f

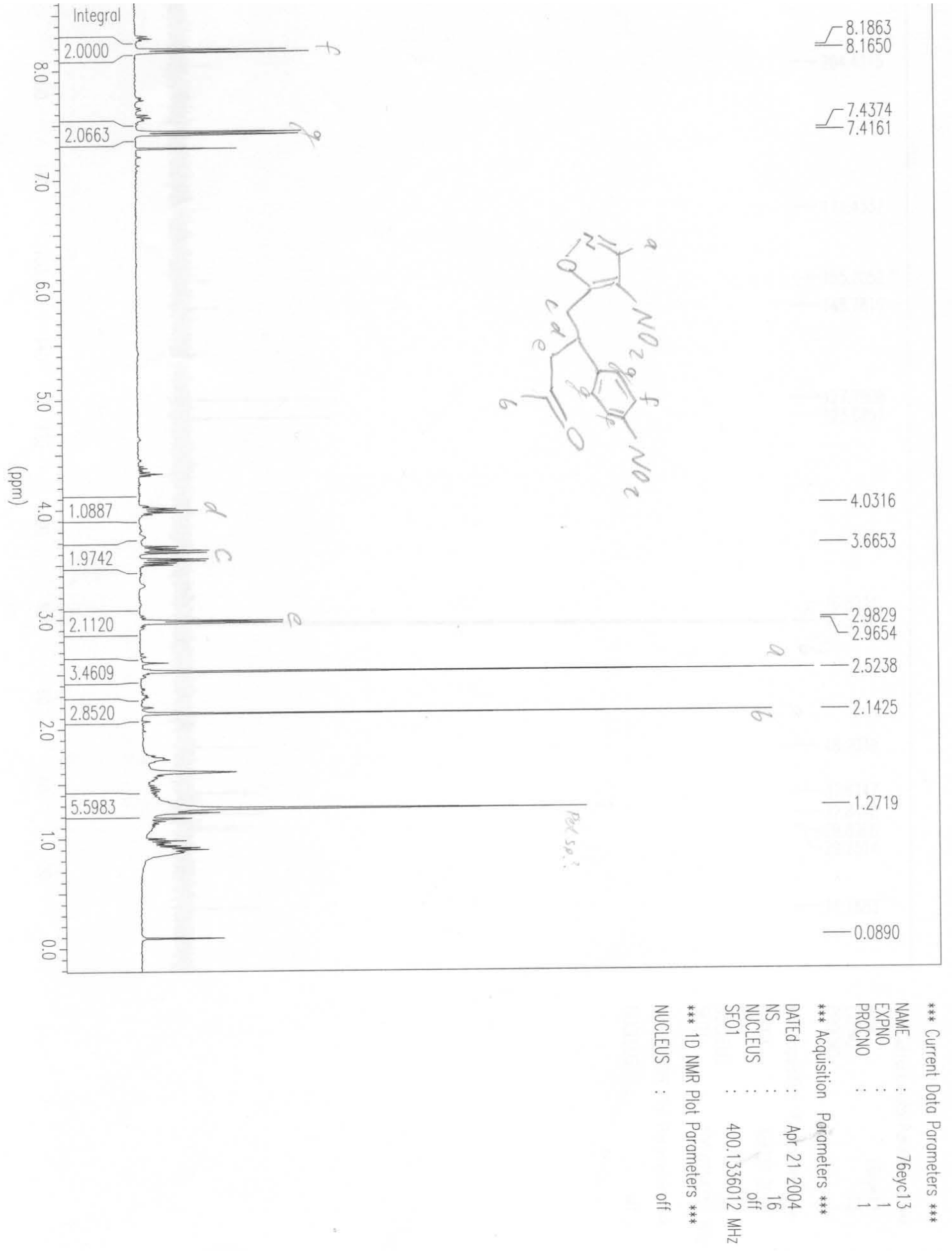


3-[1-(4-Chloro-phenyl)-2-(3-methyl-4-nitro-isoxazol-5-yl)-ethyl]-pentane-2,4-dione 3g

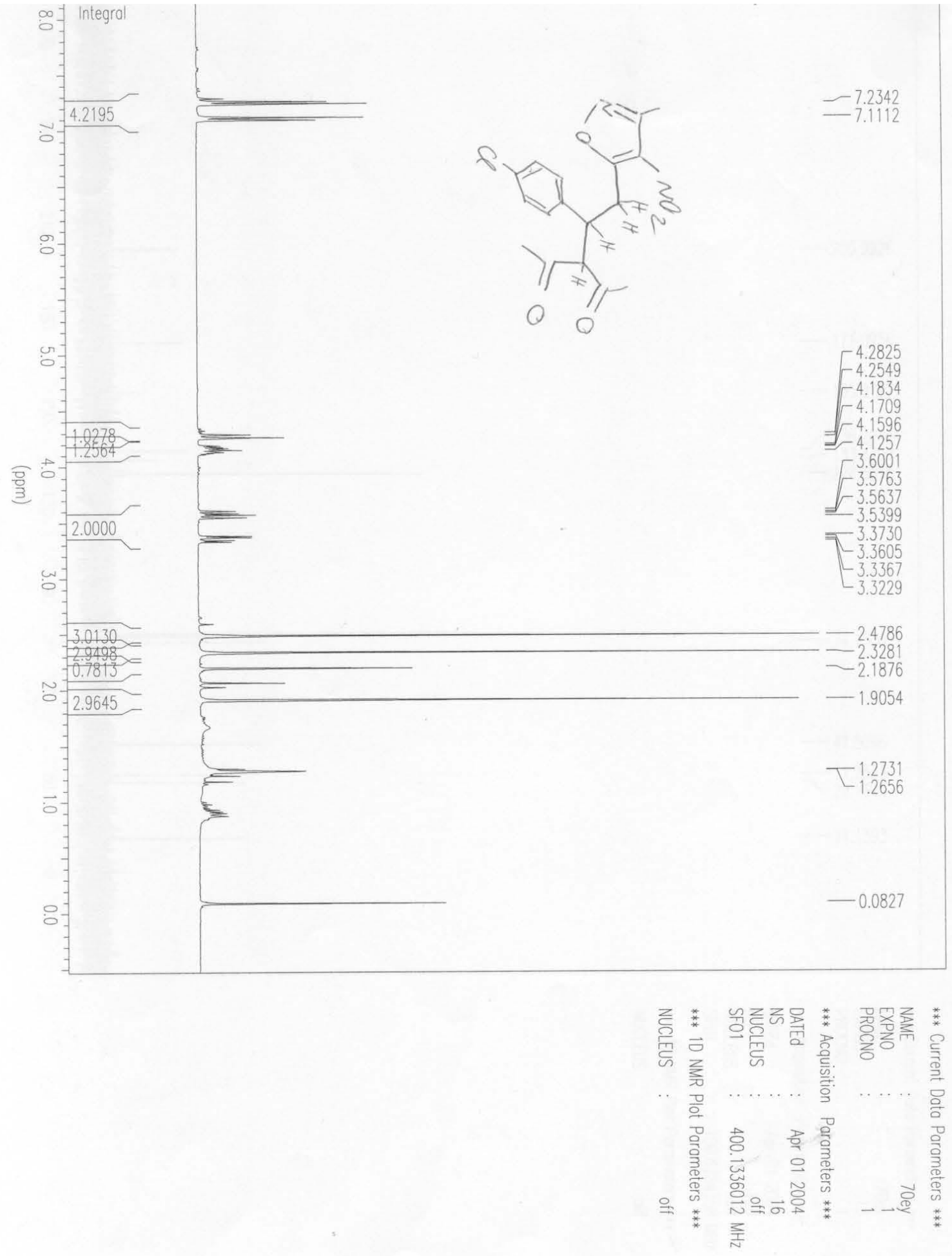


2-Acetyl-3-(4-chloro-phenyl)-4-(3-methyl-4-nitro-isoxazol-5-yl)-butyric acid ethyl ester $3 \mathrm{~h}$

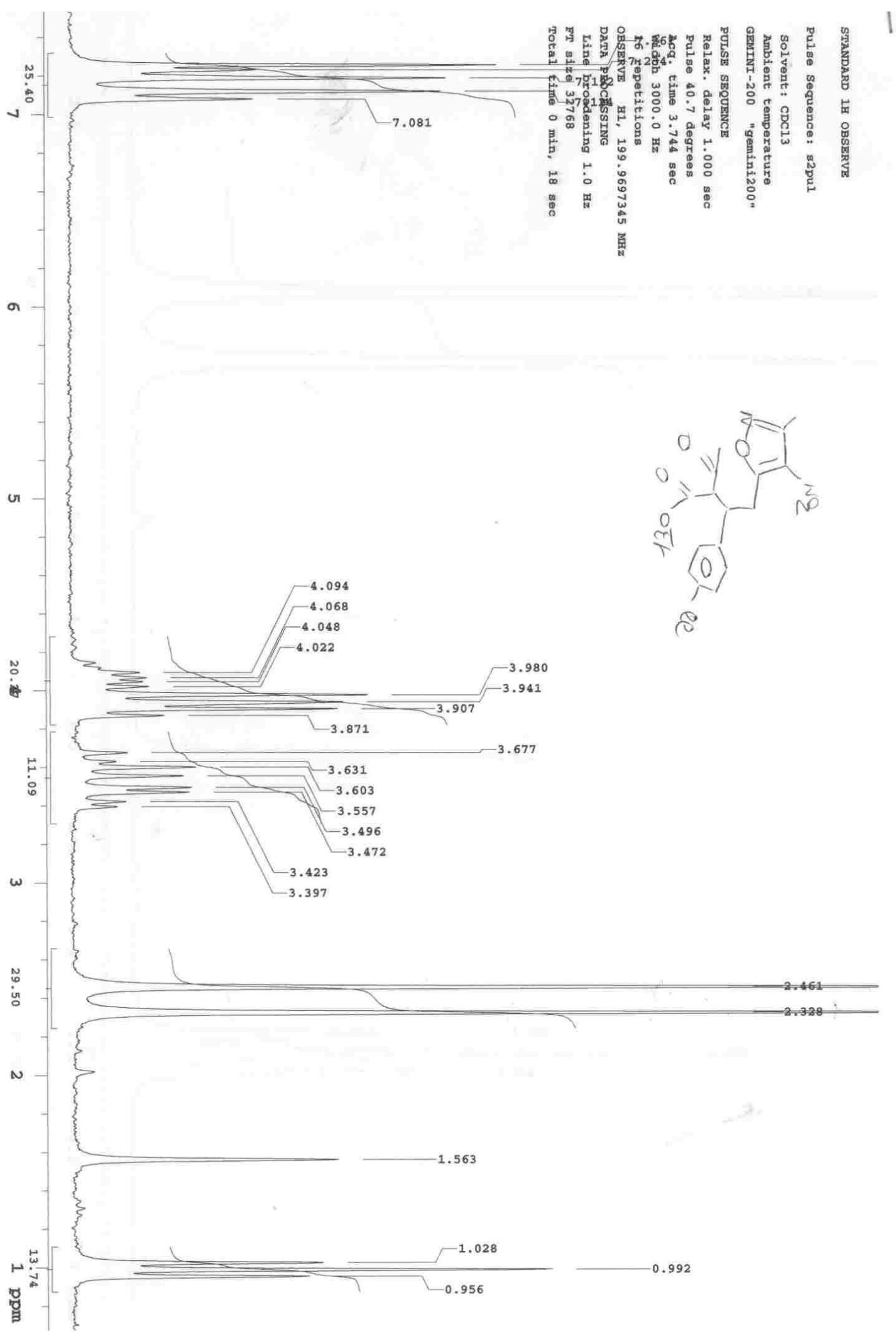




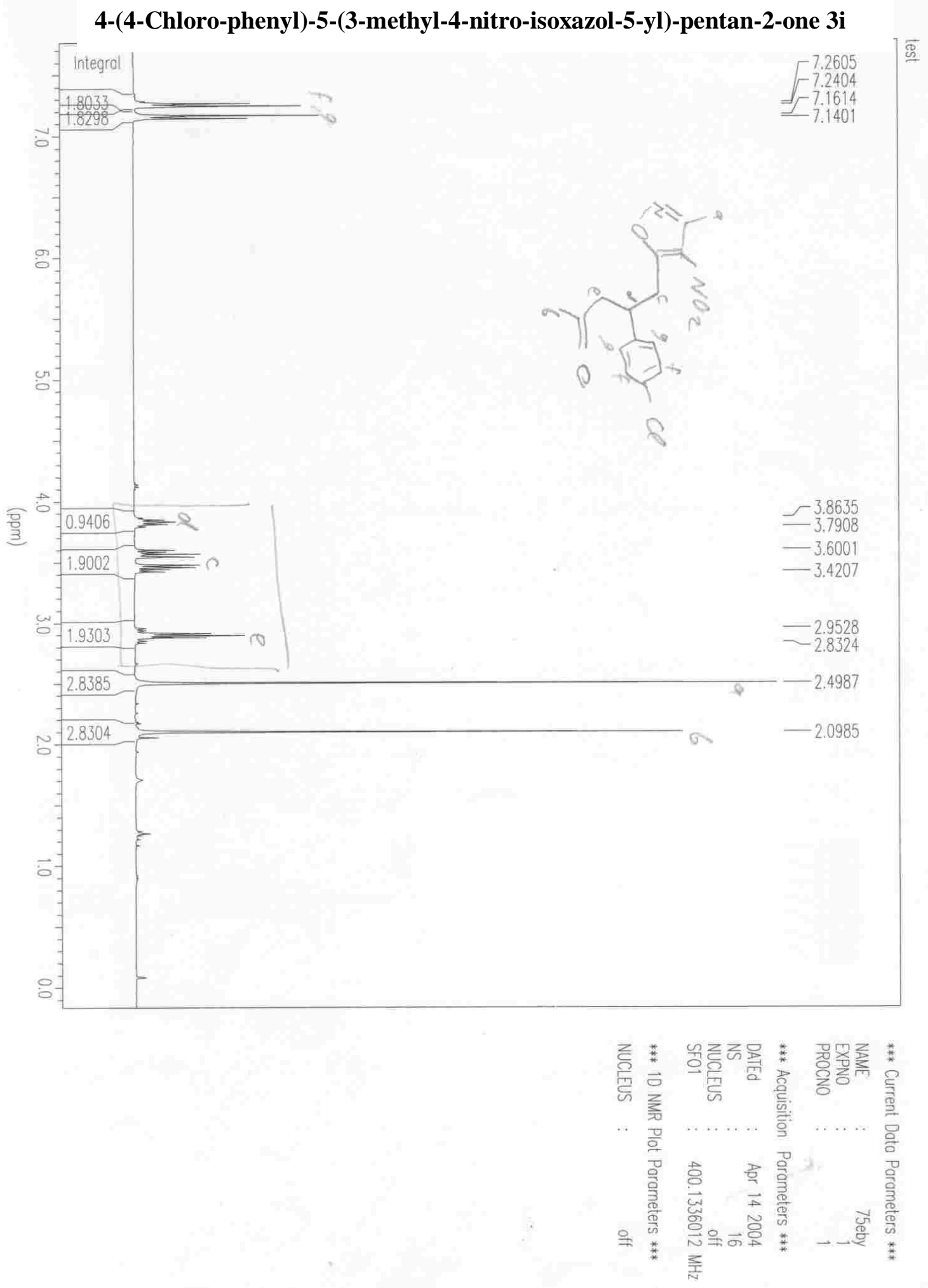


3-[1-(2-Chloro-phenyl)-2-(3-methyl-4-nitro-isoxazol-5-yl)-ethyl]-pentane-2,4-dione 3k

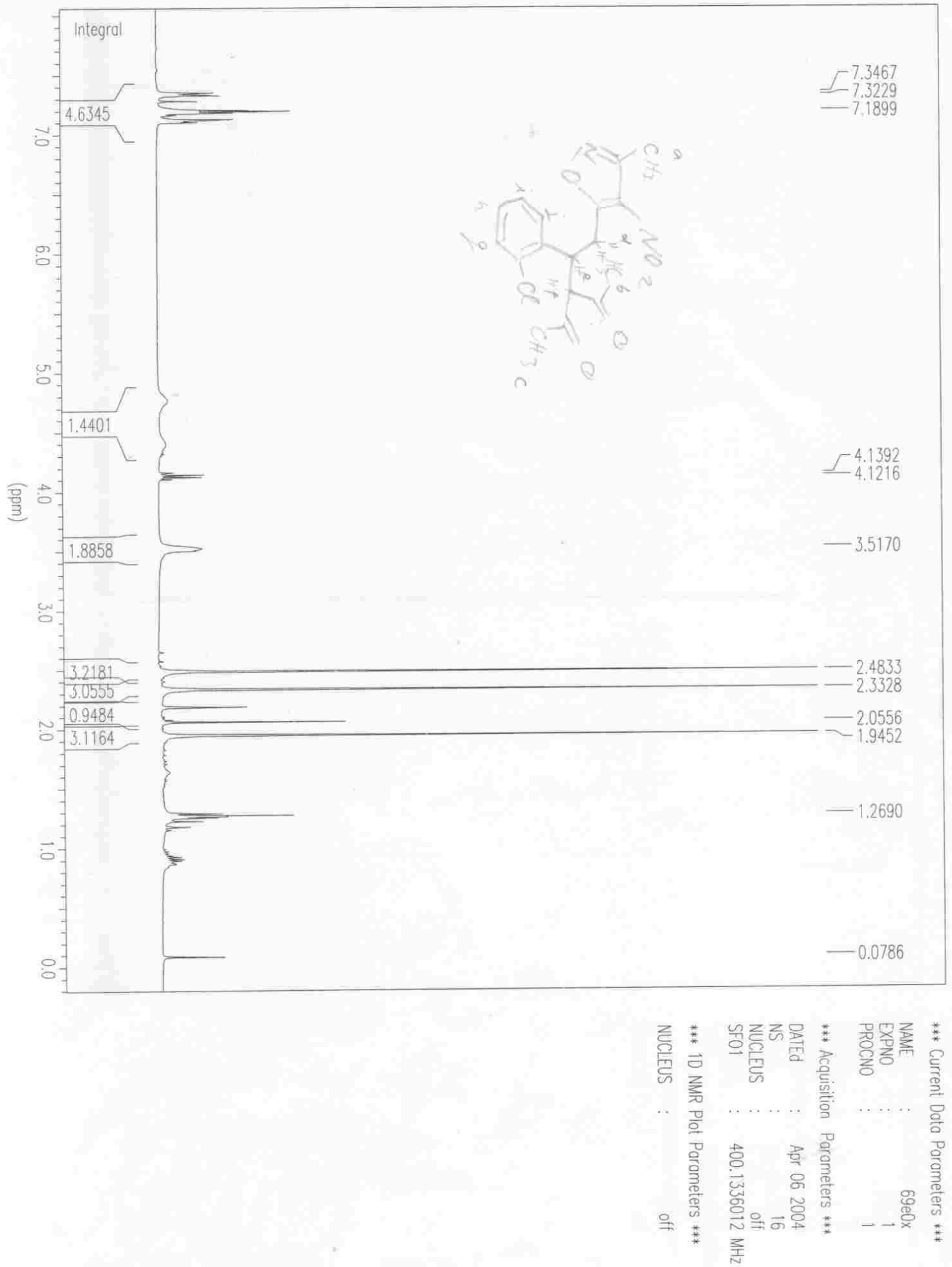


2-Acetyl-3-(2-chloro-phenyl)-4-(3-methyl-4-nitro-isoxazol-5-yl)-butyric acid ethyl ester 3l

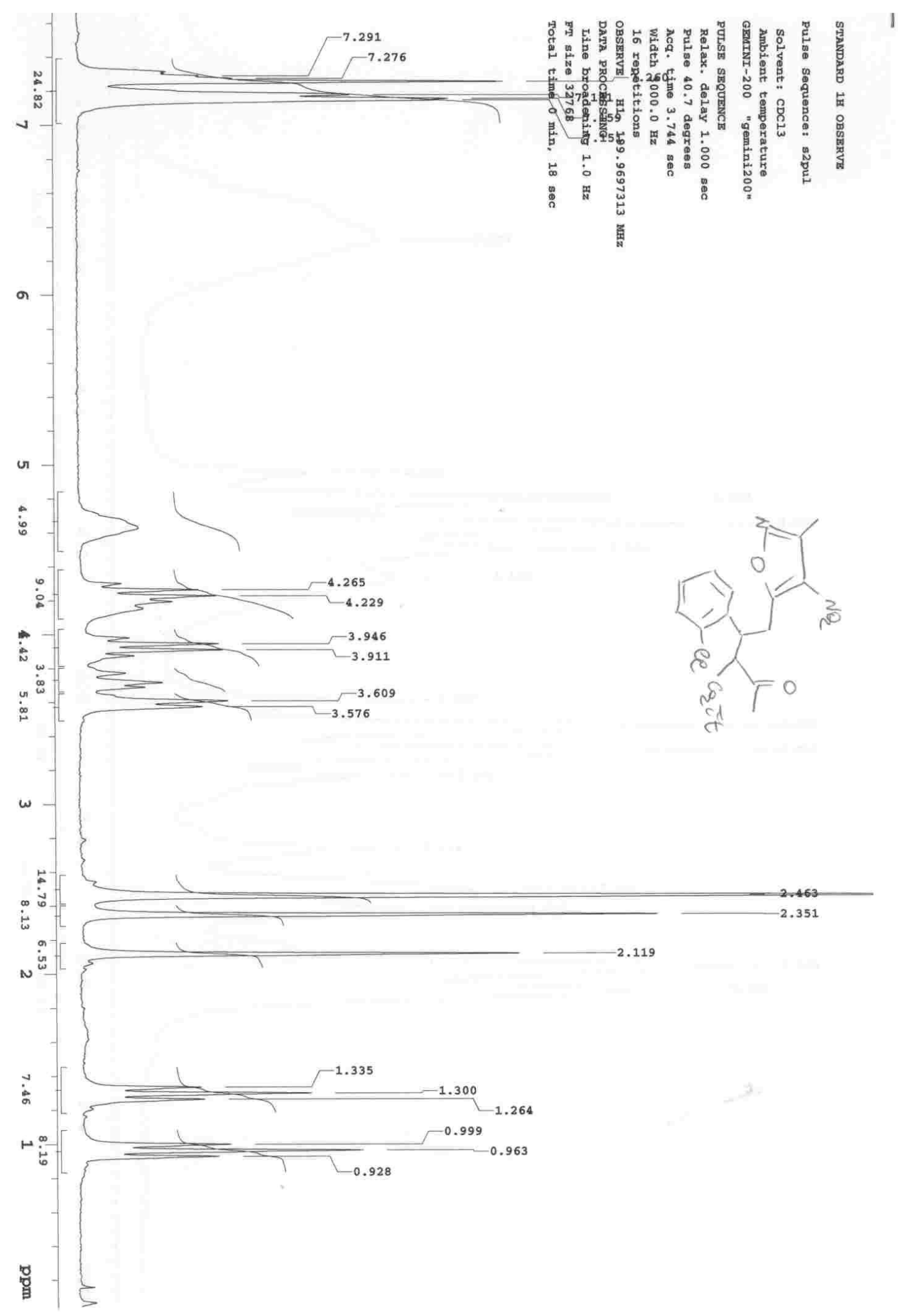


4-(2-Chloro-phenyl)-5-(3-methyl-4-nitro-isoxazol-5-yl)-pentan-2-one 3m

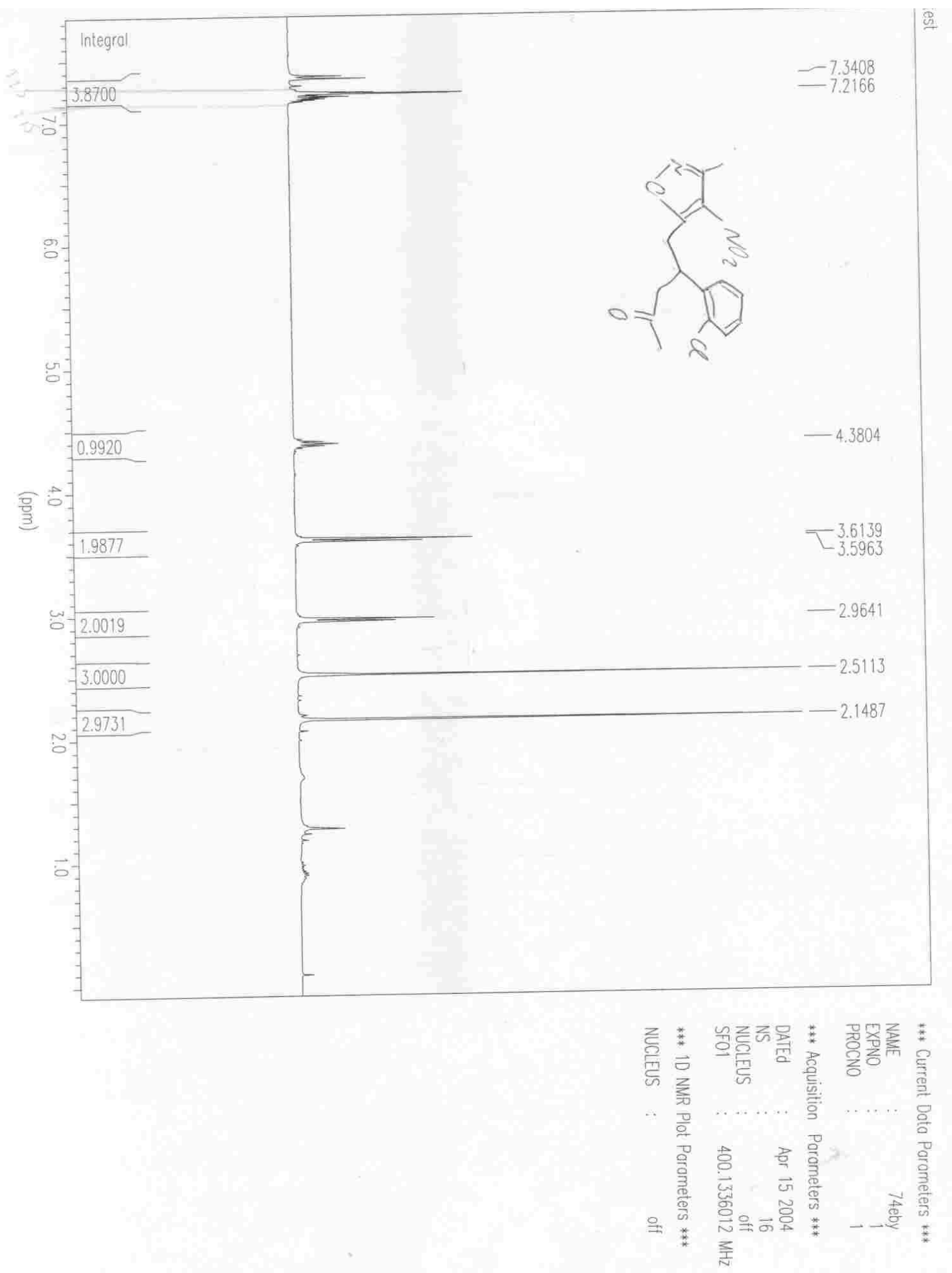


NMR Peak Assignments for 5a and 4-epi-5a: $\delta_{\mathrm{H}}\left(400 \mathrm{MHz}, \mathrm{CD}_{3} \mathrm{COCD}_{3}\right)$ for 5a: 7.41-7.23 (5H, m,

$\underline{\mathrm{Ph}}), 6.02\left(1 \mathrm{H}, \mathrm{s}, \mathrm{CHNO}_{2}\right), 4.43(1 \mathrm{H}, \mathrm{d}, J=12, \mathrm{O}=\mathrm{CC} \underline{\mathrm{HC}}=\mathrm{O}), 3.79(1 \mathrm{H}, \mathrm{td}, J=12, J=4, \mathrm{CHPh}), 3.24$ $\left(1 \mathrm{H}, \mathrm{d}, J=14, \mathrm{C}_{\mathrm{ax}} \mathrm{H}_{\mathrm{eq}} \mathrm{C}=\mathrm{O}\right), 2.73\left(1 \mathrm{H}, \mathrm{dd}, \mathrm{CH}_{\mathrm{ax}} \underline{\mathrm{H}}_{\mathrm{eq}} \mathrm{C}=\mathrm{O}, J=12, J=2.5\right) 2.63(1 \mathrm{H}, \mathrm{t}, J=12$, $\left.\mathrm{C}_{\mathrm{ax}} \mathrm{H}_{\mathrm{eq}} \mathrm{CHPh}\right), 2.02\left(3 \mathrm{H}, \mathrm{s} \mathrm{C} \underline{H}_{3} \mathrm{C}=\mathrm{N}\right), 1.88\left(3 \mathrm{H}, \mathrm{s}, \mathrm{CH}_{3} \mathrm{C}=\mathrm{O}\right), 1.89(1 \mathrm{H}, \mathrm{ddd}, J=12, J=4, J=2.5$, $\left.\mathrm{CH}_{\mathrm{ax}} \underline{\mathrm{H}}_{\mathrm{eq}} \mathrm{CHPh}\right) ; \delta_{\mathrm{H}}\left(400 \mathrm{MHz}, \mathrm{CD}_{3} \mathrm{COCD}_{3}\right)$ for 4-epi-5a: 7.41-7.23 (5H, m, $\left.\underline{\mathrm{Ph}}\right), 5.88\left(1 \mathrm{H}, \mathrm{s}, \mathrm{CHNO}_{2}\right)$, $4.43(1 \mathrm{H}, \mathrm{d}, J=12, \mathrm{O}=\mathrm{CC} \underline{\mathrm{HC}}=\mathrm{O}), 3.79(1 \mathrm{H}, \mathrm{td}, J=12, J=4, \mathrm{C} \underline{\mathrm{Ph}}), 3.28(1 \mathrm{H}, \mathrm{d}, J=14$, $\left.\mathrm{C}_{\mathrm{H}_{\mathrm{ax}}} \mathrm{H}_{\mathrm{eq}} \mathrm{C}=\mathrm{O}\right), 2.52\left(1 \mathrm{H}, \mathrm{t}, J=12, \underline{\mathrm{C}}_{\mathrm{ax}} \mathrm{H}_{\mathrm{eq}} \mathrm{CHPh}\right), 2.37\left(1 \mathrm{H}, \mathrm{dd}, J=12, J=2.5, \mathrm{CH}_{\mathrm{ax}} \underline{\mathrm{H}}_{\mathrm{eq}} \mathrm{C}=\mathrm{O}\right), 2.18$ $\left(1 \mathrm{H}, \mathrm{ddd}, J=12, J=4, J=2.5, \mathrm{CH}_{\mathrm{ax}} \underline{\mathrm{H}}_{\mathrm{eq}} \mathrm{CHPh}\right), 2.02\left(3 \mathrm{H}, \mathrm{s} \underline{\mathrm{C}}_{3} \mathrm{C}=\mathrm{N}\right), 1.88\left(3 \mathrm{H}, \mathrm{s}, \mathrm{C}_{3} \mathrm{C}=\mathrm{O}\right) ; \delta_{\mathrm{c}}$ (80Mhz, $\left.\mathrm{CD}_{3} \mathrm{COCD}_{3}\right) 202.7(\underline{\mathrm{C}}=\mathrm{O}), 201.9$ and $201.8(\underline{\mathrm{C}}=\mathrm{O}), 151.2$ and $151.0\left(\mathrm{CH}_{3} \underline{\mathrm{C}}=\mathrm{N}\right), 141.2(\mathrm{Ph})$, $128.3(\mathrm{Ph}), 127.8(\mathrm{Ph}), 126.9$ and $126.8(\mathrm{Ph}), 97.8$ and $\left.97.5(\underline{\mathrm{C} H N O})_{2}\right), 88.1\left(\mathrm{O}_{2} \mathrm{NCHCO}\right), 66.5$ and 66.4 $(\mathrm{O}=\mathrm{C} \underline{\mathrm{CHC}}=\mathrm{O}), 48.6$ and $44.6\left(\underline{\mathrm{CH}}_{2} \mathrm{C}=\mathrm{O}\right), 41.0$ and $35.9\left(\underline{\mathrm{CH}}_{2} \mathrm{CHPh}\right), 37.5$ and $36.9(\underline{\mathrm{C} H P h}), 29.8$ $\left(\underline{\mathrm{CH}}_{3} \mathrm{C}=\mathrm{O}\right), 10.7\left(\underline{\mathrm{CH}}_{3} \mathrm{C}=\mathrm{N}\right)$.

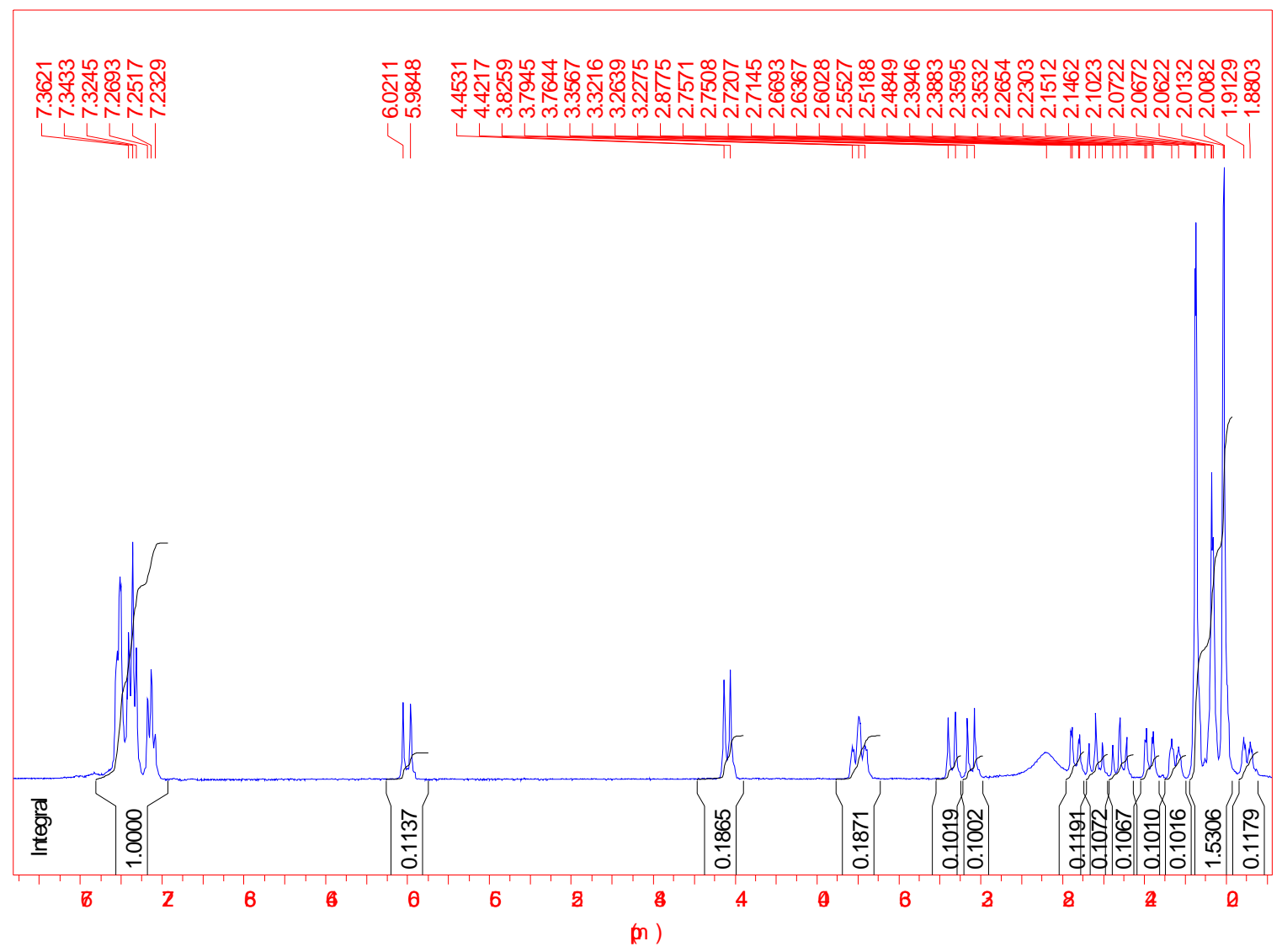




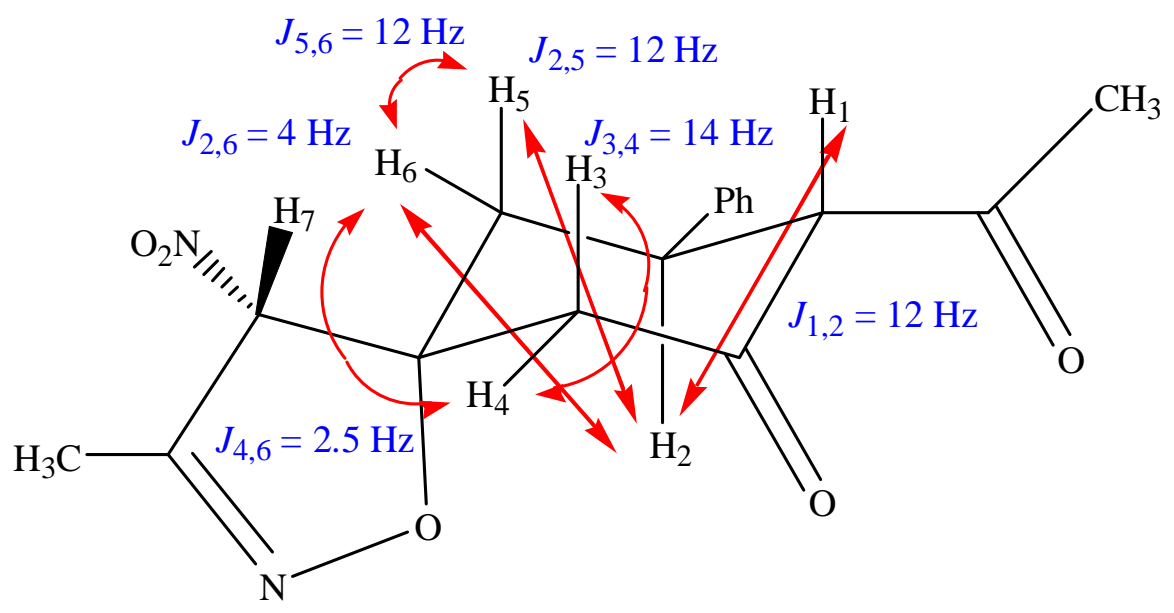

Figure 1: coupling between protons $\mathrm{H}_{1}-\mathrm{H}_{6}$

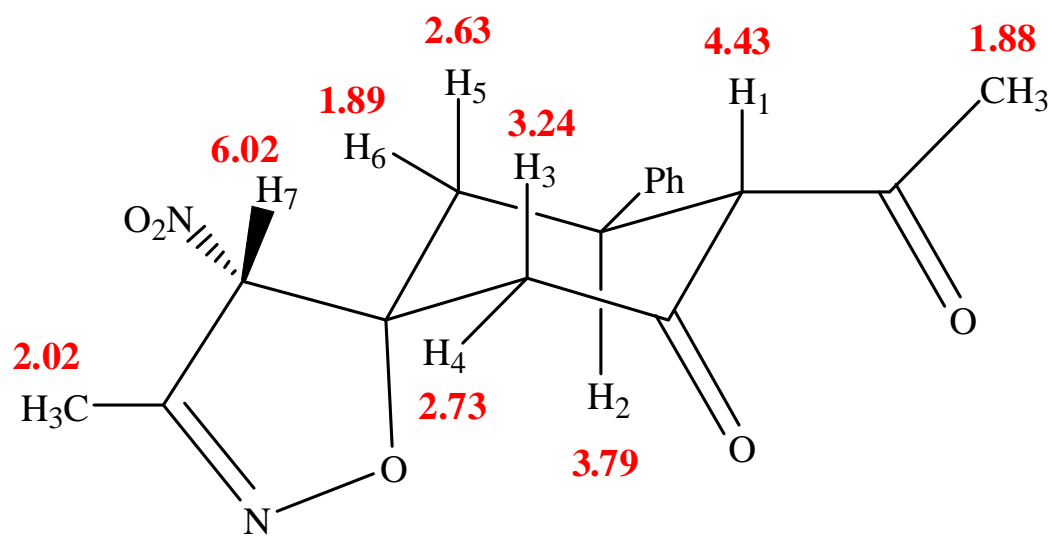

Figure 2: ${ }^{1} \mathrm{H}$-NMR chemical shifts of $5 \mathrm{a}$

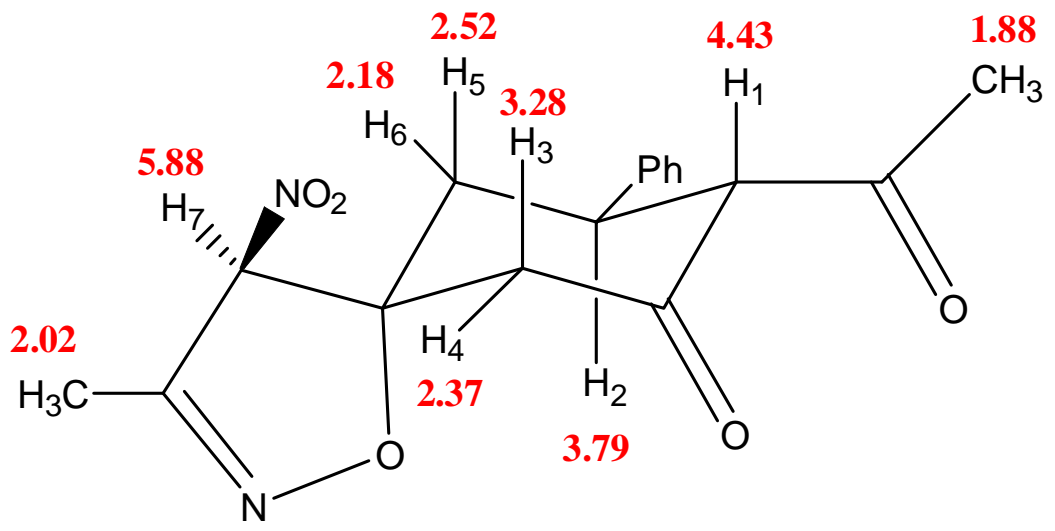

Figure 3: ${ }^{1} \mathrm{H}-\mathrm{NMR}$ chemical shifts of 4-epi- 5a 


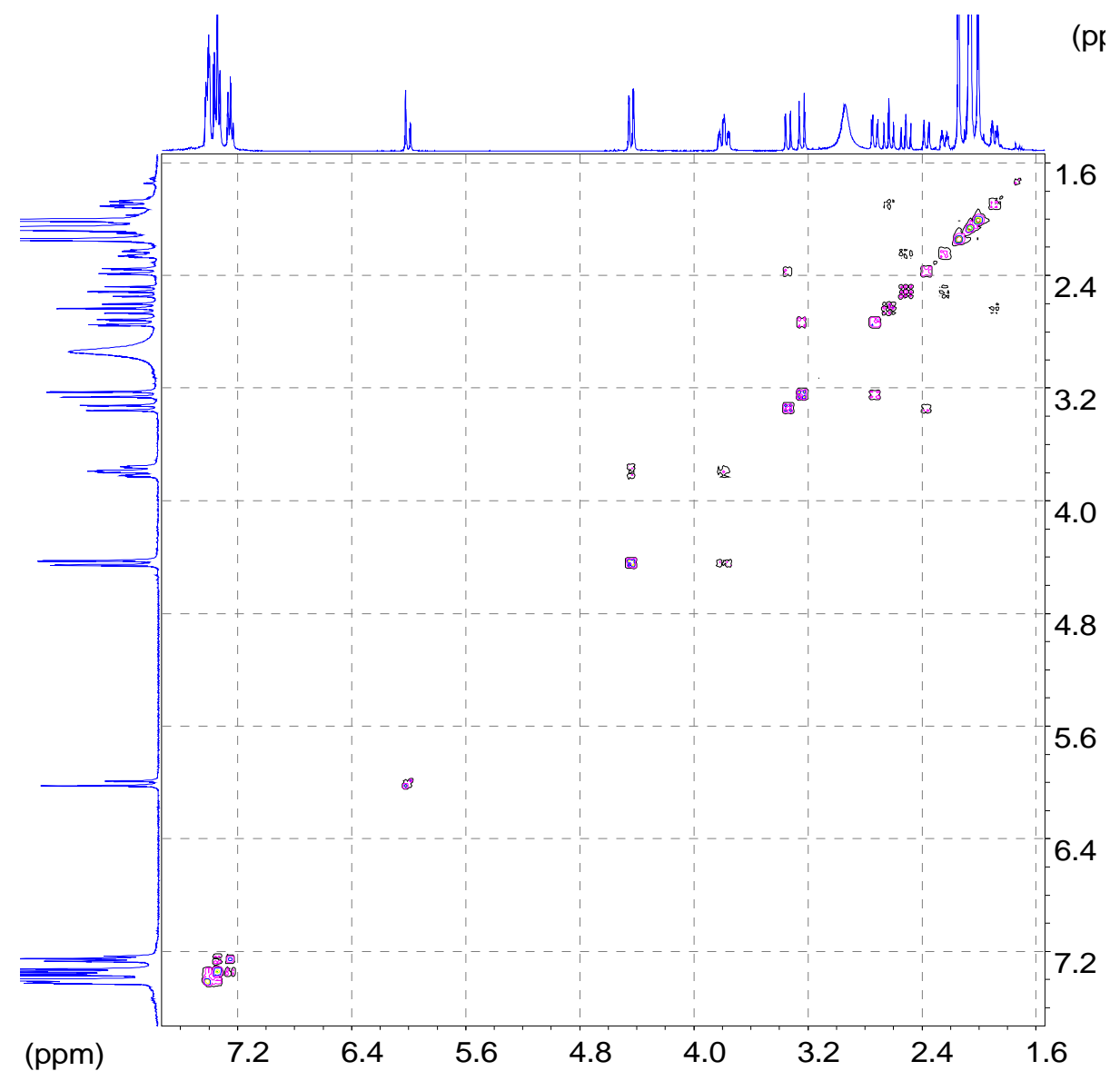

${ }^{1} \mathrm{H}-{ }^{1} \mathrm{H}$ correlation for compound $5 \mathrm{a}$ 


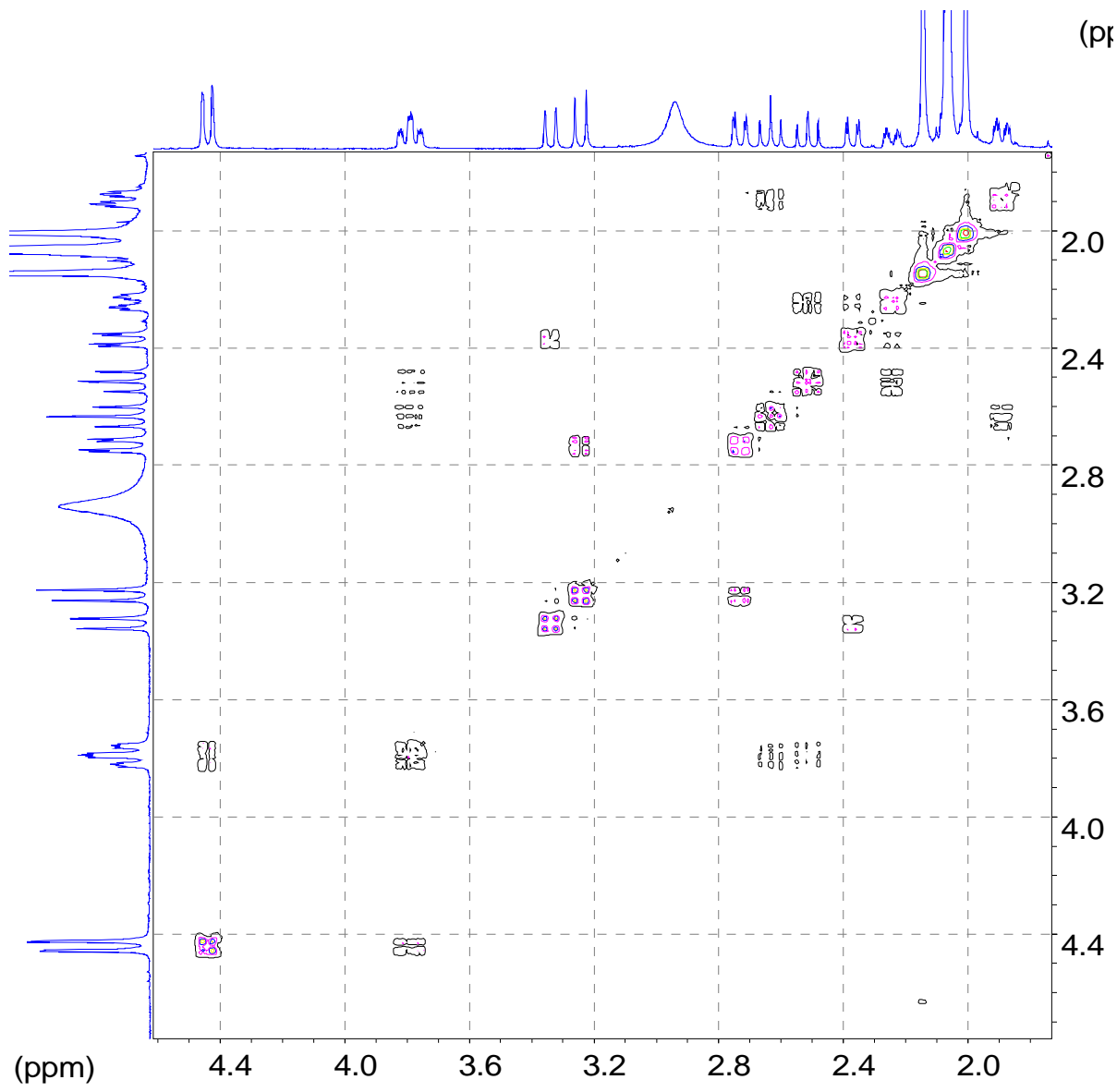

${ }^{1} \mathrm{H}-{ }^{1} \mathrm{H}$ correlation for compound $5 \mathrm{a}$ 

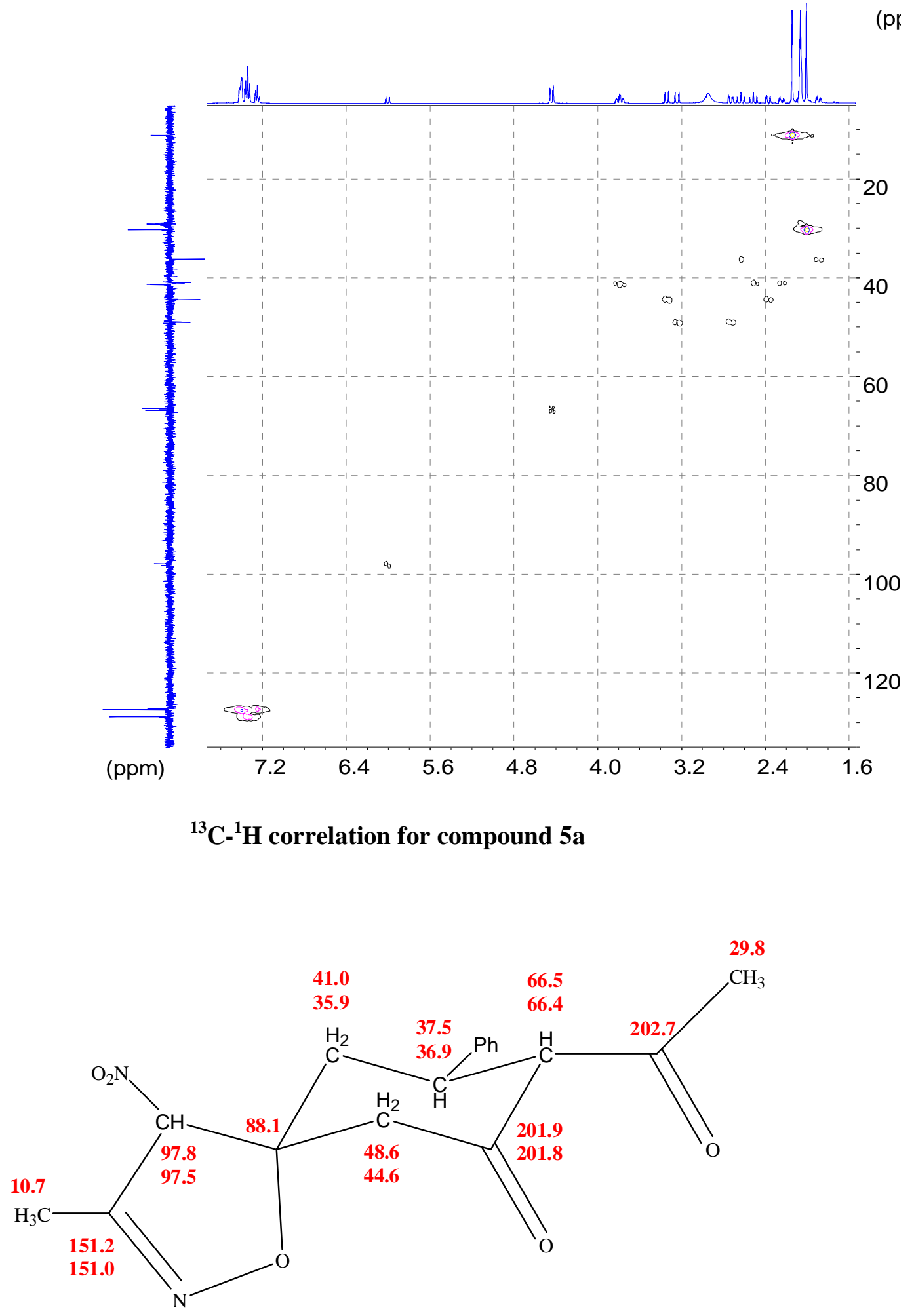

Figure 4: ${ }^{13} \mathrm{C}-\mathrm{NMR}$ chemical shifts of 4-epi- 5a 


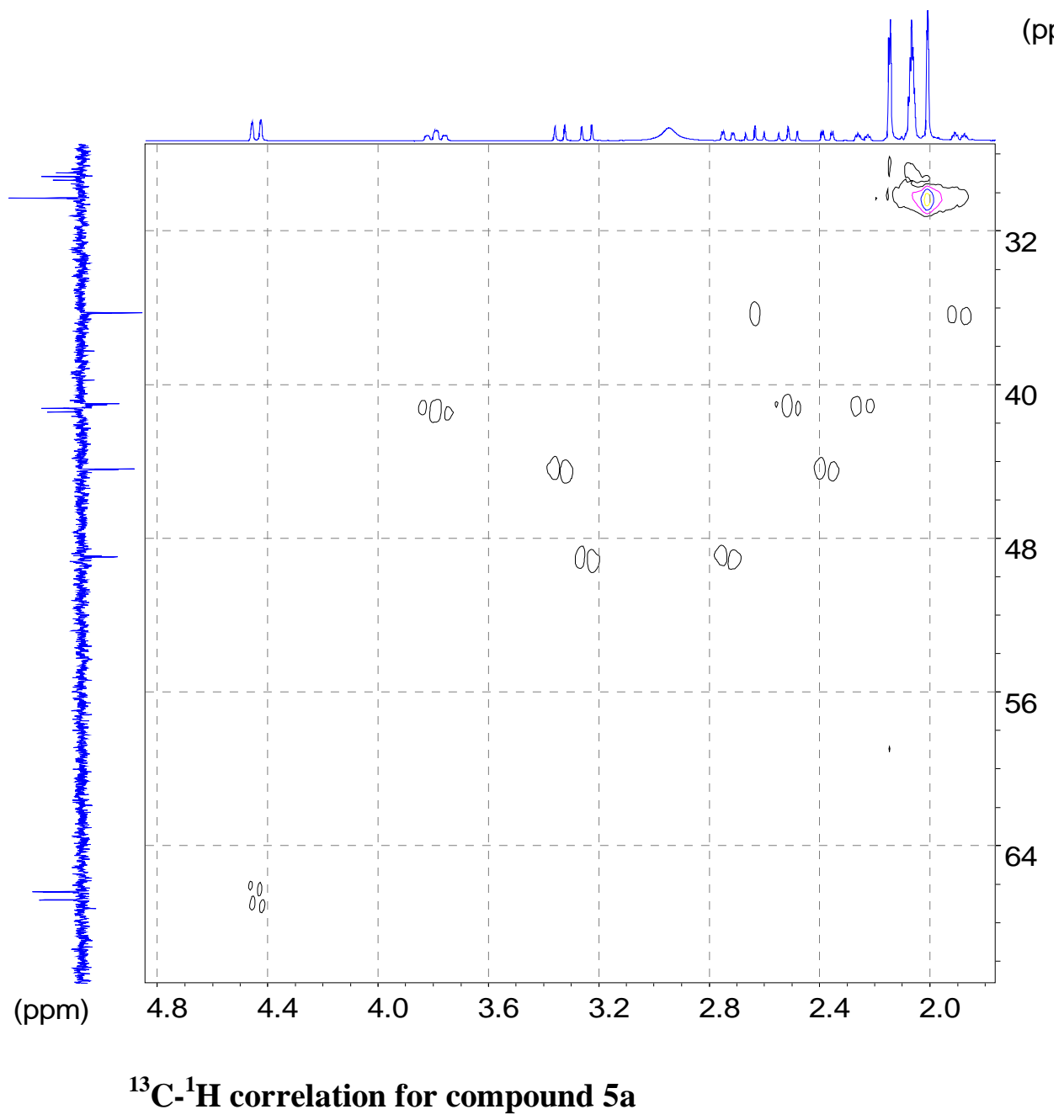


n.O.e. experiments for the assignment of 5a and 4-epi-5a (reference n. 9)

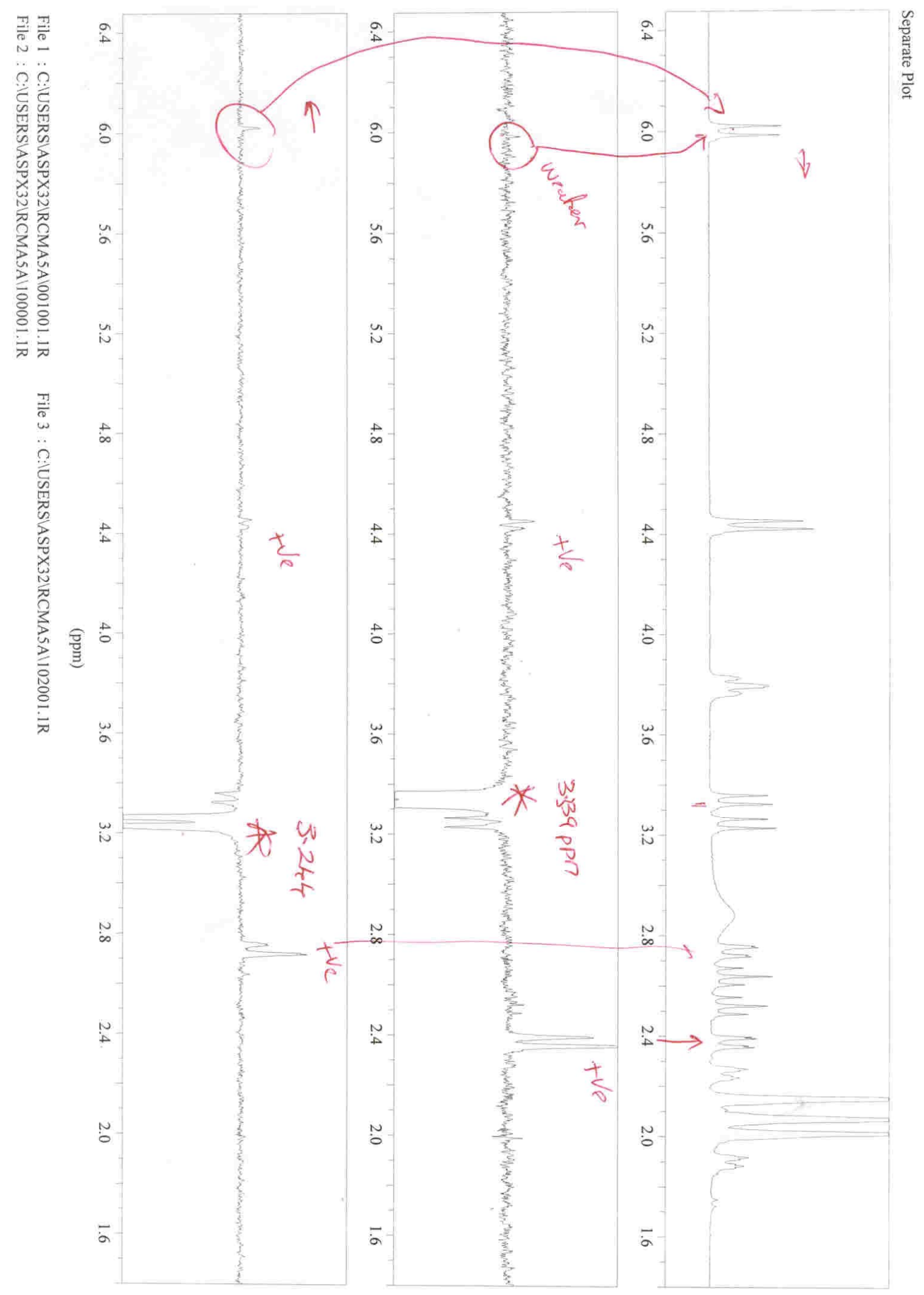

